\title{
When Face-to-Face Communication Fails: A Case Study of WVU Extension Agents Utilizing Innovative Social Media Practices
}

\author{
Madison Brooke Quinn \\ mbq0001@mix.wvu.edu
}

Follow this and additional works at: https://researchrepository.wvu.edu/etd

Part of the University Extension Commons

\section{Recommended Citation}

Quinn, Madison Brooke, "When Face-to-Face Communication Fails: A Case Study of WVU Extension Agents Utilizing Innovative Social Media Practices" (2021). Graduate Theses, Dissertations, and Problem Reports. 8048.

https://researchrepository.wvu.edu/etd/8048

This Thesis is protected by copyright and/or related rights. It has been brought to you by the The Research Repository @ WVU with permission from the rights-holder(s). You are free to use this Thesis in any way that is permitted by the copyright and related rights legislation that applies to your use. For other uses you must obtain permission from the rights-holder(s) directly, unless additional rights are indicated by a Creative Commons license in the record and/ or on the work itself. This Thesis has been accepted for inclusion in WVU Graduate Theses, Dissertations, and Problem Reports collection by an authorized administrator of The Research Repository @ WVU. For more information, please contact researchrepository@mail.wvu.edu. 
When Face-to-Face Communication Fails: A Case Study of WVU Extension Agents Utilizing Innovative Social Media Practices

Madison Brooke Quinn

\begin{abstract}
Thesis submitted
to the Davis College of Agriculture, Natural Resources, and Design at West Virginia University
\end{abstract}

in partial fulfillment of the requirements for the degree of

Master of Science in

Agricultural and Extension Education

\author{
Haley Rosson, Ph.D., Chair \\ Aaron Giorgi, Ph.D. \\ Jason McKibben, Ph.D.
}

Department of Design and Community Development

\title{
Morgantown, West Virginia
}

2021

Keywords: Extension, Innovative social media practices, failed communication

Copyright 2021 Madison Brooke Quinn 


\author{
ABSTRACT \\ When Face-to-Face Communication Fails: A Case Study of WVU Extension Agents \\ Utilizing Innovative Social Media Practices
}

\title{
Madison Brooke Quinn
}

An important aspect that contributes to the success of county Extension programs is relationships and collaborations with community members. This became a challenge when faceto-face communication was no longer possible as a response to the national pandemic that occurred due to COVID-19. In recent years, society has been increasing their reliability on social networking for communication (Diem et al., 2011). Social media played a central role in the diffusion of information in a variety of organizations and businesses during COVID-19 (Geol et al., 2020). The purpose of this study was to describe how WVU Extension Service is utilizing innovative social media practices to remain connected with clientele when face-to-face communication was not possible. The opportunity to research this alternative method of communication was the response to protocols enacted by executive orders to slow the spread of COVID-19. Case study methodology was used for this study and West Virginia Extension agents utilizing innovative social media practices were interviewed. Results from the study described (1) innovative social media platforms being used, (2) how innovative social media practices are being used, (3) why innovative social media practices are being used, and (4) perceptions and needs related to utilizing innovative social media practices. 


\section{ACKNOWLEDGEMENTS}

I am extremely fortunate to have such influential and supportive people in my life. I would like to begin by thanking my parents. It's safe to say that without my Mom and Dad, I wouldn't be the person I am today. Thank you for always believing in me and pushing me to do my best every day. You all have showed me the value of hard work and determination, which I have carried with me during my collegiate career. I would also like to thank my pup, Riley. You always know how to cheer me up when I'm feeling stressed and overwhelmed.

I cannot thank my advisor and committee chair, Dr. Haley Rosson, enough for making my graduate school experience truly remarkable. You have guided me and encouraged me with my studies and my research. The endless opportunities and resources you have shared with me has not gone unnoticed. Thank you for being one of my biggest supporters throughout graduate school. I'm very grateful to have a role model like you.

Thank you to Dr. Jason McKibben and Dr. Aaron Giorgi for being a part of my committee as well. You both have pushed me to succeed and supported me through my research and graduate school. I appreciate the many conversations and thought-provoking questions that led me to becoming a better researcher and professional student.

Having a support system of colleagues, who have become my close friends, has been a blessing. My graduate cohort, Travis Veach, Katie Daughterty, Cole Payne, and Kindra Carr, have been an absolute pleasure to work with. You all have impacted my graduate school experience in a very positive way. Thank you for the endless office laughs, study dates, and group chat memes. I would also like to thank two of my very best friends, Jamie Hetrick and Mikayla Hargis, who also studied Agricultural and Extension Education at WVU. You both have encouraged me and supported me, which I cannot thank you enough for. 
There are many other individuals who I am extremely grateful for who have supported me and led me to where I am today. I am truly blessed by all of those in my life and appreciate the positive encouragement. 


\section{TABLE OF CONTENTS}

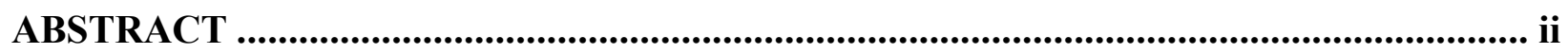

ACKNOWLEDGEMENTS .............................................................................................. ii

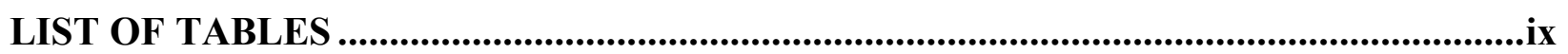

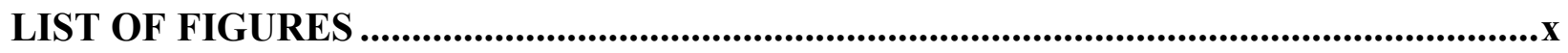

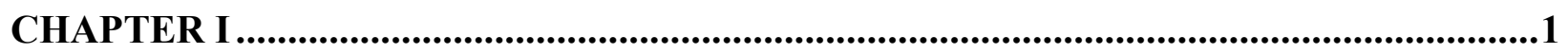

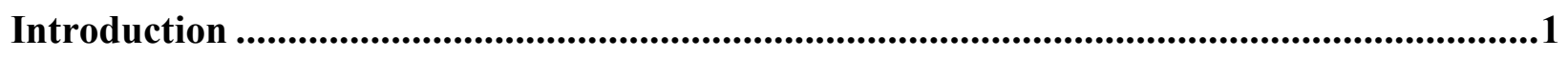

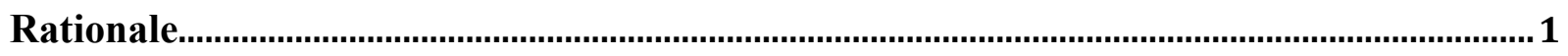

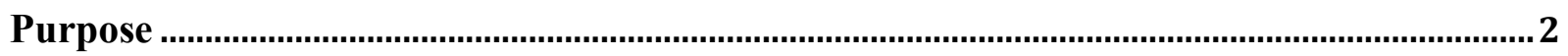

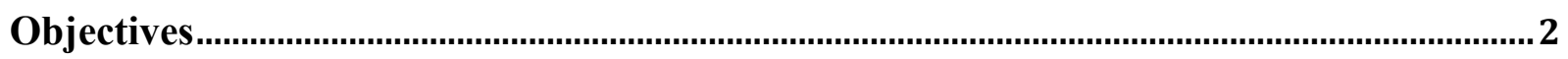

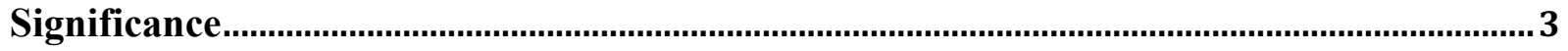

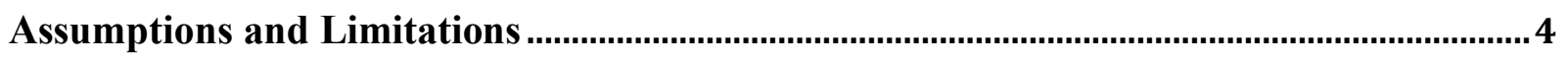

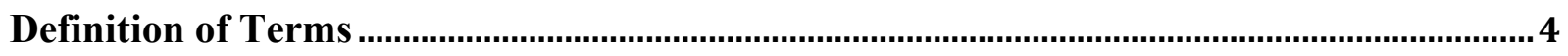

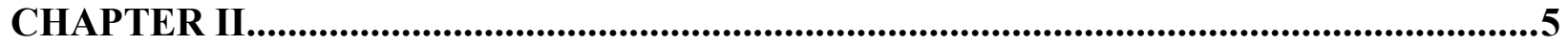

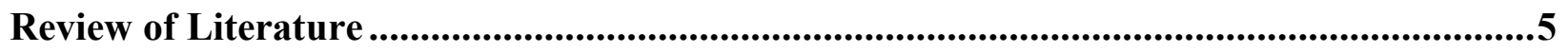

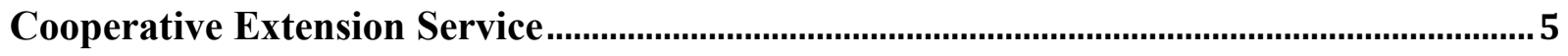

WVU Extension Service.....................................................................................

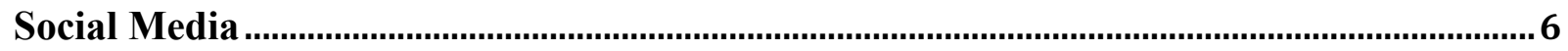

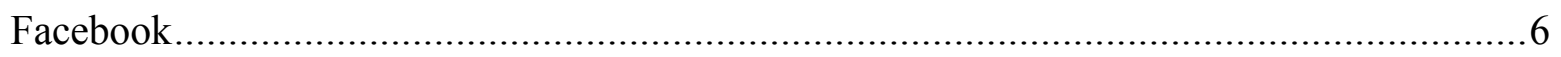

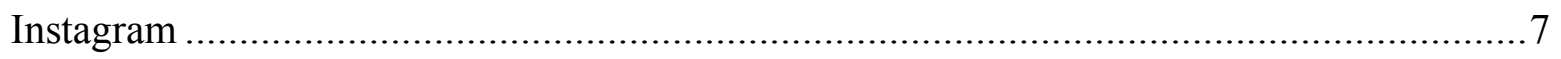

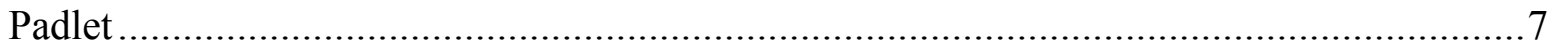

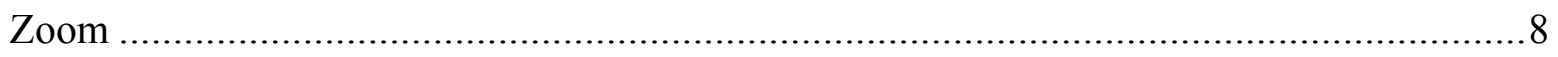

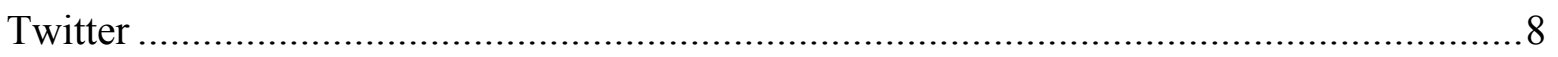

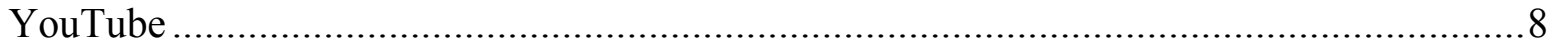

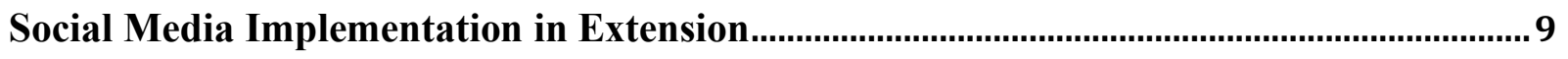

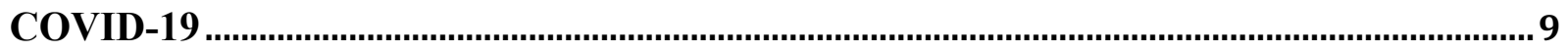

Social Media in Times of COVID-19

Extension During COVID-19................................................................................................... 10

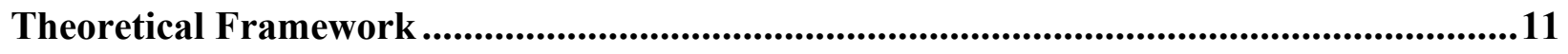




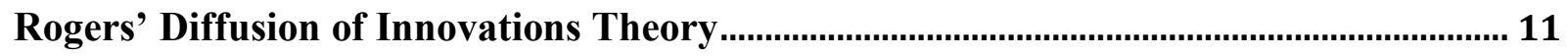

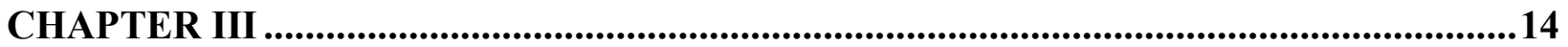

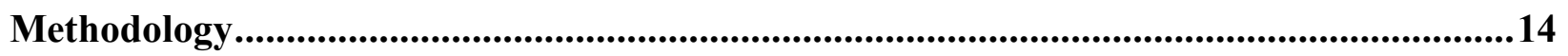

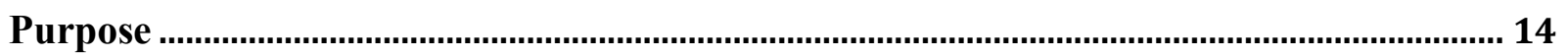

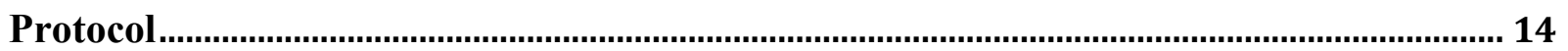

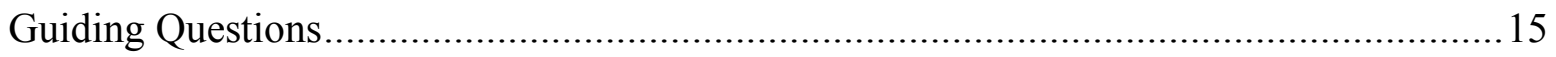

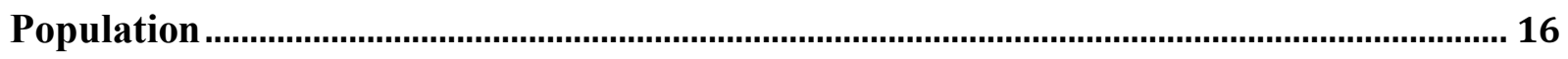

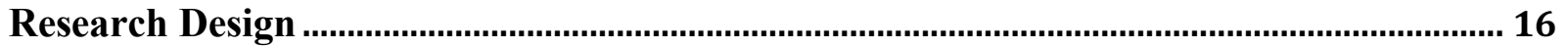

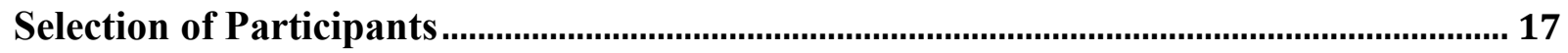

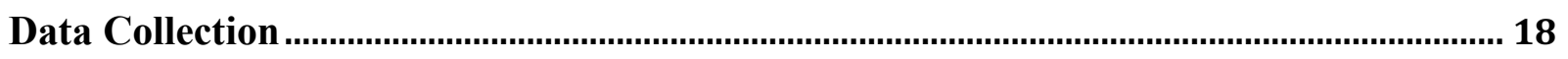

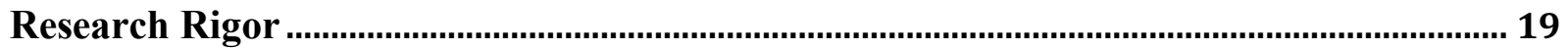

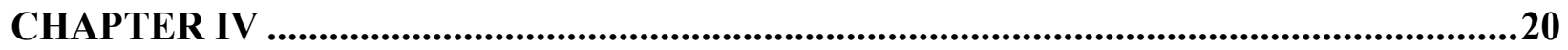

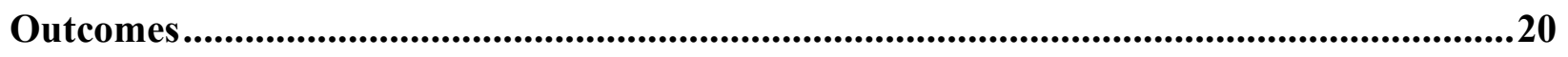

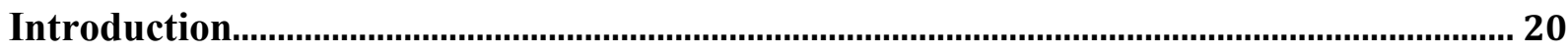

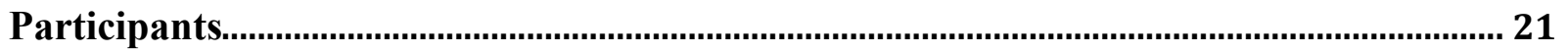

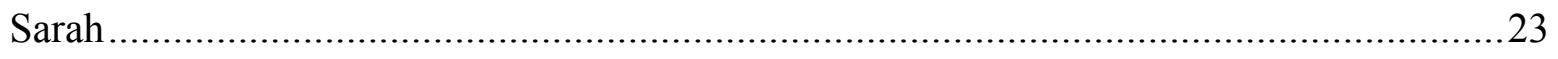

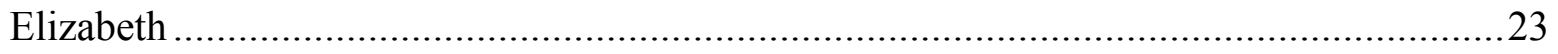

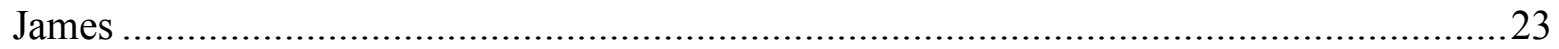

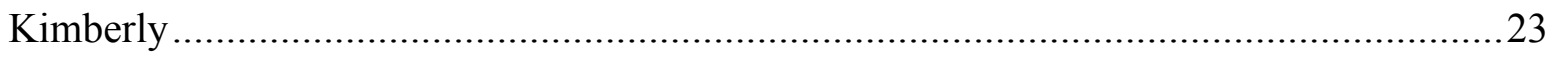

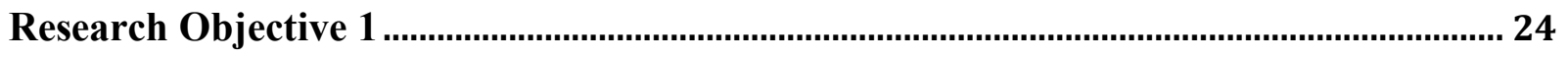

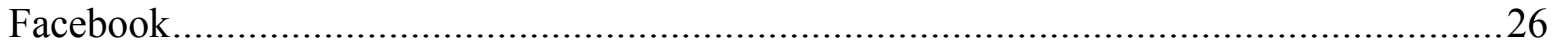

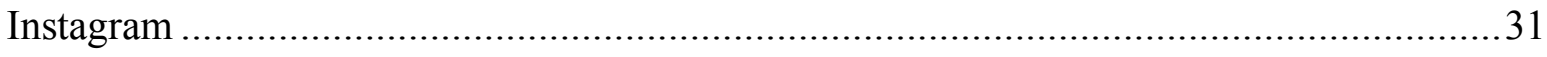

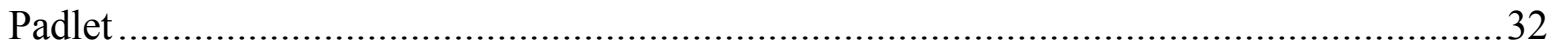

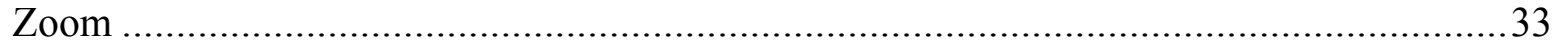

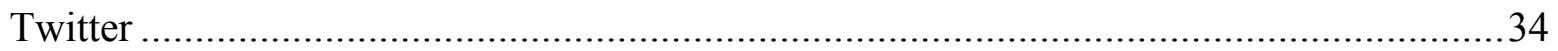

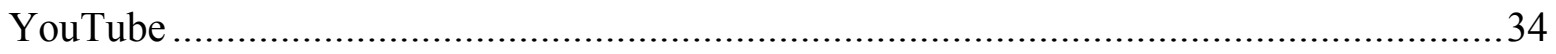

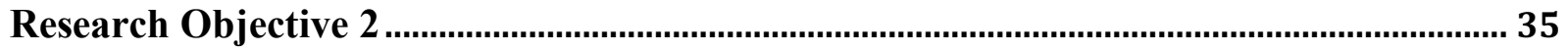

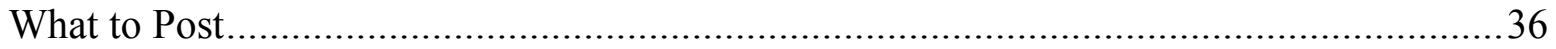

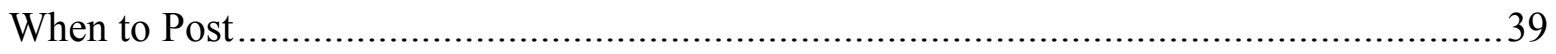


Research Objective 3 ....................................................................................................... 41

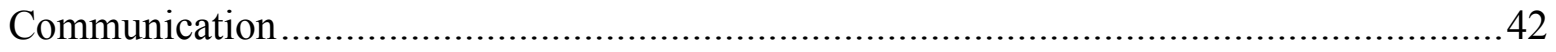

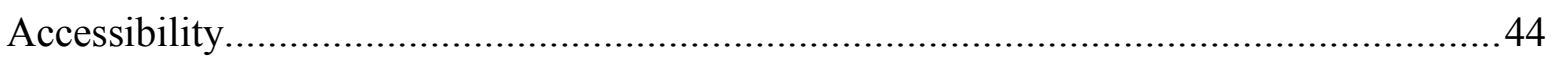

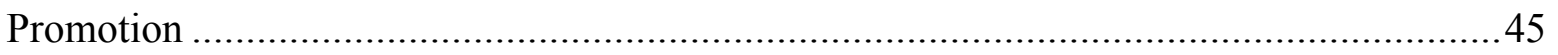

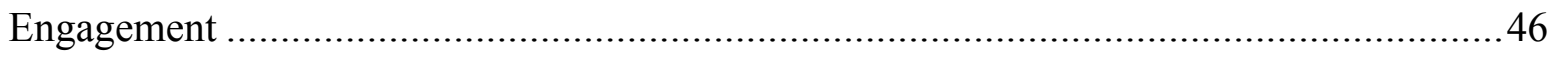

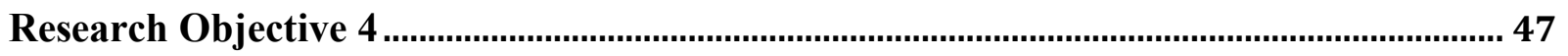

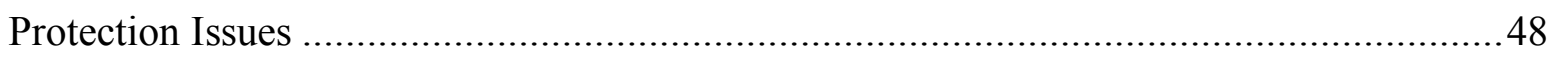

Separating Work from Personal Life ……………….................................................

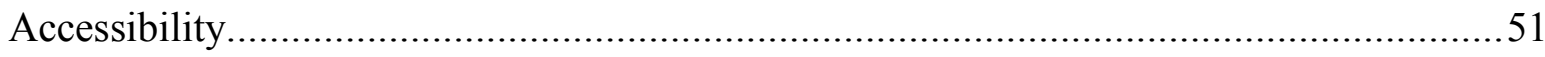

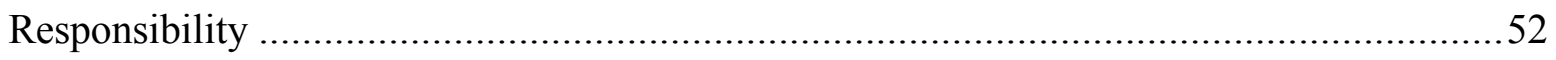

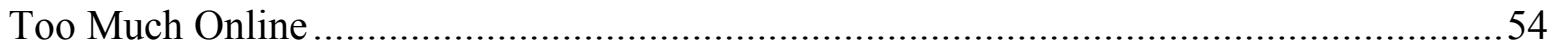

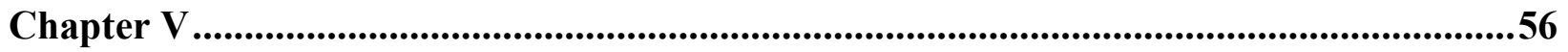

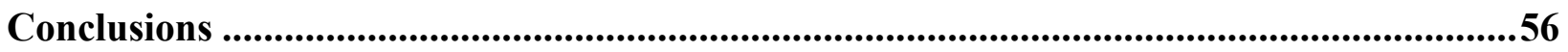

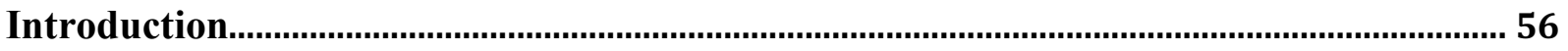

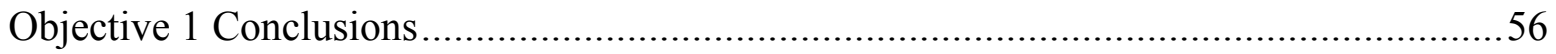

Objective 2 Conclusions ...................................................................................... 59

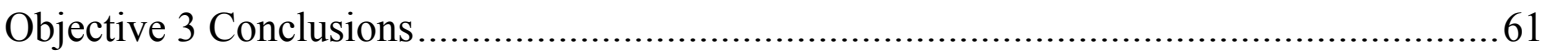

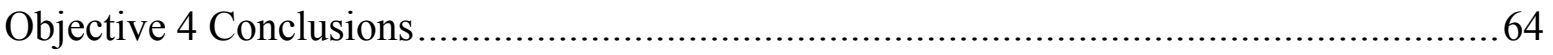

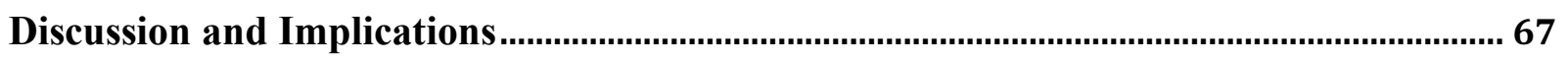

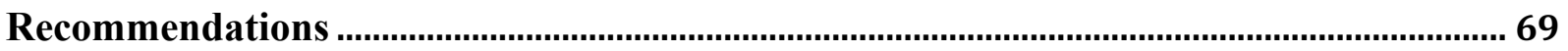

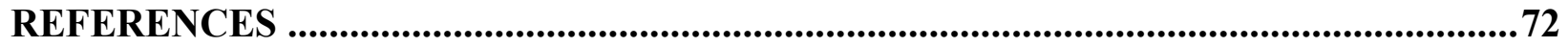

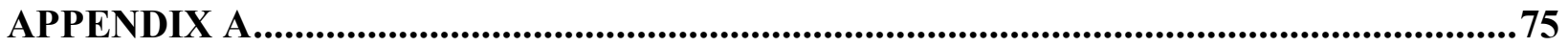

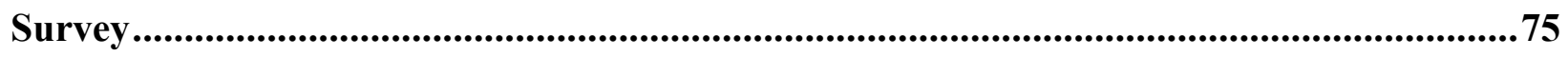

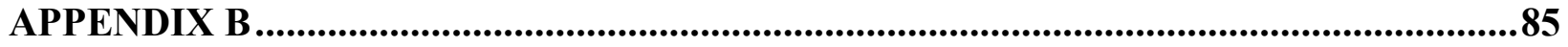

Interview Guiding Questions...................................................................................................85

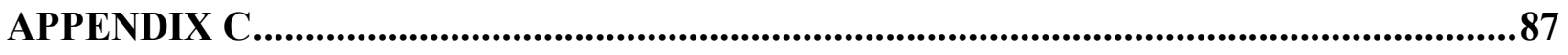

Transcribed Interviews ....................................................................................................................8

Sarah

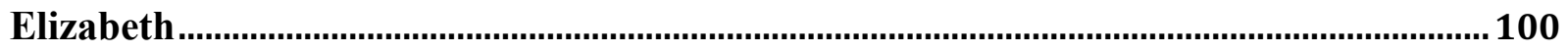

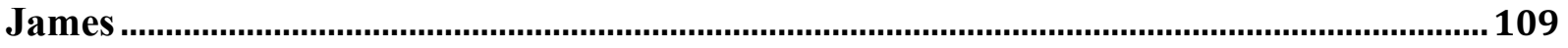




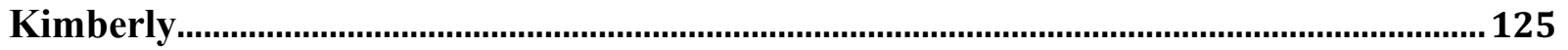

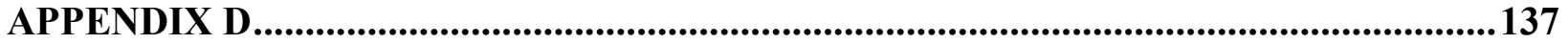

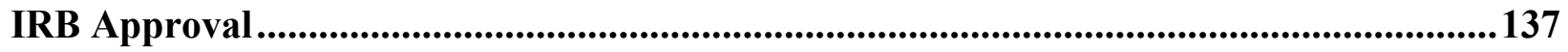




\section{LIST OF TABLES}

4.1 Participant Program Unit Information................................... 30

4.2 Social Media Platform Usage by Participant.................................. 31

4.3 Participant Social Media Usage As a Response to COVID-19....................31

4.4 Coding for How WV Extension is Using Innovative SM Platforms

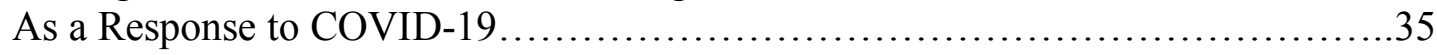

4.5 Coding for How WV Extension is Using Innovative SM Practices

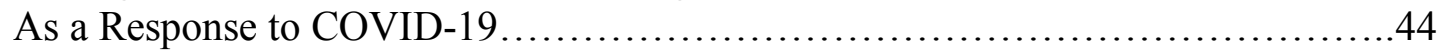

4.6 Coding for Why WV Extension is Using Innovative SM Platforms

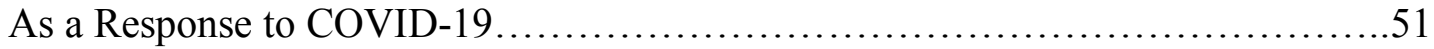

4.7 Coding for Perceptions of Barriers and Needs Related to Extension Using Innovative SM Practices As a Response to COVID-19....................57 


\section{LIST OF FIGURES}

2.1 Rogers' (2003) Adopter Categorization on the Basis of Innovation..................20

2.2 Rogers' (2003) Model of Five Stages in the Innovation-Decision Process............22

4.1 Social Media Platform Word Cloud..............................................34 


\section{CHAPTER I}

\section{Introduction}

The national pandemic that occurred due to COVID-19 beginning in March of 2020 resulted in a shutdown of many services within the United States. This occurrence was unexpected and sudden for the general population within the United States. Many services, including West Virginia University (WVU) Extension, had to close offices and work remotely. This meant that the Extension Service, which relied heavily on face-to-face communication, was now expected to work with their clientele at a distance. This new means of communication was something that the WVU Extension service had not experienced before.

Important factors in contributing to a successful county Extension program are the relationships and collaborations with members of the community. These vital relationships have become increasingly difficult to maintain under the current circumstances and regulations established to protect citizens from COVID-19. County Extension programs within WVU Extension have been working diligently to remain connected with community members during this difficult time. Progressive means for maintaining connection with clientele began to emerge in WVU Extension.

\section{Rationale}

In recent years, society has been shifting to an increasing amount of reliability on social networking for communication (Diem et al., 2011). Communication through social media in Extension has been explored in many different studies due to this increase. Several studies have examined how social media complimented and assisted with pre-existing communication 
methods (Cornelisse et al., 2011; Diem et al., 2011; Gharis, 2011). However, it is also important to consider how social media has been implemented in Extension when other methods, such as in-person communication, are suddenly not possible anymore. Social media now plays a central role in the diffusion of information in a variety of fields as a response to the global pandemic of COVID-19 (Geol et al., 2020).

\section{Purpose}

The purpose of this study was to describe how WVU Extension Service is utilizing innovative social media practices to remain connected with clientele when face-to-face communication was not possible. The opportunity to research this alternative method of communication is the response to protocols enacted by executive orders to slow the spread of COVID-19. Knowledge and understanding of this information can assist West Virginia Extension Service, especially extension agents, with maintaining connection with their clientele. This is beneficial presently because protocols are still in place to slow the spread of COVID-19. This is also beneficial in other circumstances when face-to-face communication fails or is limited.

\section{Objectives}

In order to achieve the purpose of the study, the following objectives were created:

1. Describe innovative social media platforms being used.

2. Describe how innovative social media practices are being used.

3. Describe why innovative social media practices are being used. 
4. Identity perceptions of barriers and needs related to utilizing innovative social media practices.

\section{Significance}

The information collected from completing this research is beneficial in the following ways:

1. Aid in establishing innovative social media practices that can be used by WVU Extension to maintain connection with clientele; and

2. Aid in understanding the significant impact social media practices within WVU Extension can have when face-to-face communication methods have failed

Extension emphasizes creating positive changes by sharing knowledge gained through research and education with community members (USDA-NIFA, 2020). Therefore, effective communication methods have a significant role in the success of Extension programs. Understanding past and current methods of communication within Extension programs will aid in the development and success of communication in the future. Recognizing and evaluating effective and ineffective methods of staying connected with the community is useful. A major purpose of Extension programs is to develop relationships with the community, which is why determining and implementing effective means of maintaining connection with clientele is essential.

Although Extension programs have recently been implementing a greater amount of alternative methods of communication, many of which are internet-based, Extension still relies heavily on face-to-face communication. When in-person communication is not possible, due to protocols enacted by executive orders to slow the spread of COVID-19, the Extension service 
began to develop new ways to network with the communities they serve. Acknowledging and understanding measures taken during this time are crucial for sustaining the longevity of the future of Extension.

\section{Assumptions and Limitations}

A major assumption of this study is that every county's Extension office in the state has access to the internet. Social media platforms require the use of the internet. If Extension personnel do not have access to the internet, then it is assumed that they are not implementing social media, which can be a limitation to this study.

\section{Definition of Terms}

COVID-19 - new disease; respiratory illness that spreads from person to person (FDA, 2020) Extension Service - provides non-formal education and learning activities to individuals and communities throughout the country (USDA, 202)

Innovativeness - the degree to which an individual or other unit of adoption is relatively earlier in adopting new ideas than other members of a system (Rogers, 2003)

Pandemic - global outbreak of an infectious disease that affects a large number of people (World Health Organization, 2011)

$S M$ - Social Media 


\section{CHAPTER II}

\section{Review of Literature}

\section{Cooperative Extension Service}

The implementation of Extension practices dates back to early history in the United States when more than $50 \%$ of the population lived in rural areas and $30 \%$ of the workforce were involved in farming (Landry, 2010). However, Extension was not formalized until 1914, when the Smith-Lever Act established the Cooperative Extension Service (USDA, 2020). This rooted the partnership between Cooperative Extension and agricultural colleges organized in 1862 . The Morrill Act of 1862 established land-grant universities that would provide education for the public in agriculture, home economics and mechanic arts (Landry, 2010). The Cooperative Extension Service extends outreach programs through these land-grant universities (USDA, 2020).

\section{WVU Extension Service}

The Extension service exists within all fifty-five counties in the state of West Virginia. The three program units are: Agriculture and Natural Resources, Family and Community Development, and 4-H Youth Development. (WVU Extension Service, 2021) Each of the fiftyfive counties in West Virginia has an office with agent(s) that fill roles for all three of the program units. "WVU Extension connects people to information and knowledge that enables them to change lives and improve their communities" (WVU Extension Service, 2021).

Results of WVU Extension programs include:

1. Transforming lives through 4-H youth development; 
2. Achieving healthier lifestyles through family and health programs;

3. Supporting and revitalizing communities through workforce and economic development; and

4. Developing efficiency and management skills through agriculture and natural resource education.

(WVU Extension Service, 2021)

\section{Social Media}

Social media is recognized as an online system of communication through various platforms, such as Facebook, Twitter, Instagram, etc. These often-free internet based applications allow for the creation and exchange of information among users (Kaplan and Haenlein, 2010). This can be done by creating posts, sharing photos and videos, sending direct messages, as well as sharing and commenting on others posts. These transactions of sharing information contribute to the creation of online communities (Rauniar et al., 2014). Considering how easily accessible social media is for everyone, communication can exist from almost any location at any time (Gharis et al., 2014).

\section{Facebook}

In 2004, a social networking platform known as Facebook emerged on the internet. This platform was created by 19-year old, Mark Zuckerberg, of Harvard University as a way for students at the university to create profiles and remained connected with one another (Brugger, 2015). The platforms popularity began to spread to other universities, then to businesses and organizations, and continued to the general public. 
Over the last decade and a half, Facebook has continuously developed features and communication techniques. Facebook provides users with the ability to share a wide variety of content with others on the internet. Facebook allows users to share and comment on status updates in forms of writing, photos, and videos. It also has a private messaging platform. Users can create and/or join public/private groups and pages. This creates networking opportunities for likeminded individuals. Facebook has remained the most popular social media platform for over a decade now (Pew Research Center, 2016).

\section{Instagram}

Instagram is a social media platform that originally debuted in late 2010 (Instagram, 2021). This platform allows users to share pictures with other users. Instagram users also have the ability to make their account public or private. Instagram utilizes similar features to Facebook, such as instant messaging, hashtags, tags, and stories. A relatively new feature to Instagram that has increased user engagement is interactive features to stories, such as polls (Instagram, 2021).

\section{Padlet}

A platform that first appeared in 2012, Padlet allows communication in professional settings. Although Padlet is very simplistic in design, it has much to offer. Padlet is commonly used by schools and teachers for online classrooms (Edwards, 2020). Schools typically pay a fee to access additional features Padlet offers, but it remains completely free for students. This digital platform elicits learning and creativity through sharing photos, links, videos, and more. 
The layout and structure to Padlet is very similar to Facebook, but offers increased privacy, making it a valuable platform for a variety of businesses and organizations, such as Extension. (Padlet, 2021).

\section{Zoom}

Founded in 2011, Zoom is a platform that is used for telecommunicating. This social networking platform is available worldwide and allows individuals to participate in virtual video calls. The simplistic design makes Zoom user friendly. Zoom has a variety of features that foster positive communication, such as waiting rooms, breakout rooms, and instant messaging. This platform also has creative aspects, such as virtual green screen backgrounds, that make Zoom more enticing for users (Zoom Video Communications Inc., 2021).

\section{Twitter}

Twitter is a microblogging social media platform that first emerged in 2006. (Twitter Inc., 2021) It allows account holders to share "Tweets" in the form of text, photos, videos, and links. Twitter also has an instant messaging platform. Users can comment and share other users Tweets. This social media platform is entirely free for users. Twitter provides opportunity for communication with friends, family, and other individuals (Twitter Inc., 2021).

\section{YouTube}

The video sharing platform, YouTube, was founded in 2005 (YouTube, 2021). It is free to create an account but does have additional features that can be paid for. YouTube users can upload videos to share with other users. Additionally, users can like, share, and comment on 
other users YouTube videos. Similar to most social media platforms, you can follow other users and get updates when they post new video content. (YouTube, 2021).

\section{Social Media Implementation in Extension}

In the workplace, social media is becoming increasingly used by businesses and organizations (Rauniar et al., 2014). The integration of social media contributes by assisting with business operations and processes (Fogel, 2010). The Extension Service is one of many organizations that has been integrating social media into their professional agenda (Gharis et al., 2014). Incorporating the use of social media is beneficial because Extension can potentially reach individuals who are not previously familiar with the Extension Service (Cornelisse et al., 2011).

Social media platforms are already being used to share important information with the public regarding news, events, and decisions (Auer, 2011). The diffusion of information through social media platforms is convenient and efficient in conjunction with the use of other means of communication. Many community members expressed that they are willing to receive information electronically from Extension agents (Bardon et al., 2007).

\section{COVID-19}

According to the Centers for Disease Control and Prevention (2020), a new strain of coronavirus, commonly known as COVID-19, began spreading throughout China beginning in December 2019. By January 2020, the World Health Organization declared a global health emergency (Mayo Clinic Health System, 2020). As the virus began spreading outside of China, President Donald Trump issued national emergency declarations in March of 2020 that would 
assist in protecting U.S citizens during the spread of COVID-19 (NCSL 2020). Regulations to slow the spread of COVID-19 were determined by each state beginning in March of 2020 as well (CDC, 2020).

\section{Social Media in Times of COVID-19}

As a response to the national pandemic caused by the spread of COVID-19, various fields have increased the use of social media on a professional level (Goel., 2020). Social media platforms are more commonly being used for the dissemination of information to the public now than ever before. One study found that Twitter became a crucial tool for world leaders to share updates and information during the pandemic of COVID-19 (Rufai, 2020). Zoom also demonstrated tremendous increases as a response to COVID-19 protocol (Strauss, 2020).

\section{Extension During COVID-19}

To date, COVID-19 is still being closely monitored and regulations are in place to slow the spread of the virus and protect individuals from exposure. Due to in person contact being very limited, businesses and organizations are forced to integrate other methods of communication (Geol et al., 2020). Many businesses and organizations, such as the Extension Service, rely heavily on in person communication. How is WVU's Extension Service using innovative social media practices while adhering to the guidelines in place during COVID-19? 


\section{Theoretical Framework}

\section{Rogers' Diffusion of Innovations Theory}

Rogers' Diffusion of Innovations Theory was used as the framework for this study. Rogers (2003), defines diffusion as "the process in which an innovation is communicated through certain channels over time among the members of a social system" (p. 5). This framework was selected because the study described innovative social media practices being utilized by West Virginia Extension.

The major goal of diffusion of an innovation is to reach adoption. Adoption is defined by Rogers (2003) as "a decision to make full use of an innovation as the best course of action available" (p. 21). An individual's innovativeness, or the degree to which an individual's adoption is earlier than others, can be visualized by examining the S-shaped bell curve of adopter categories. This is shown in Figure 2.1 below.

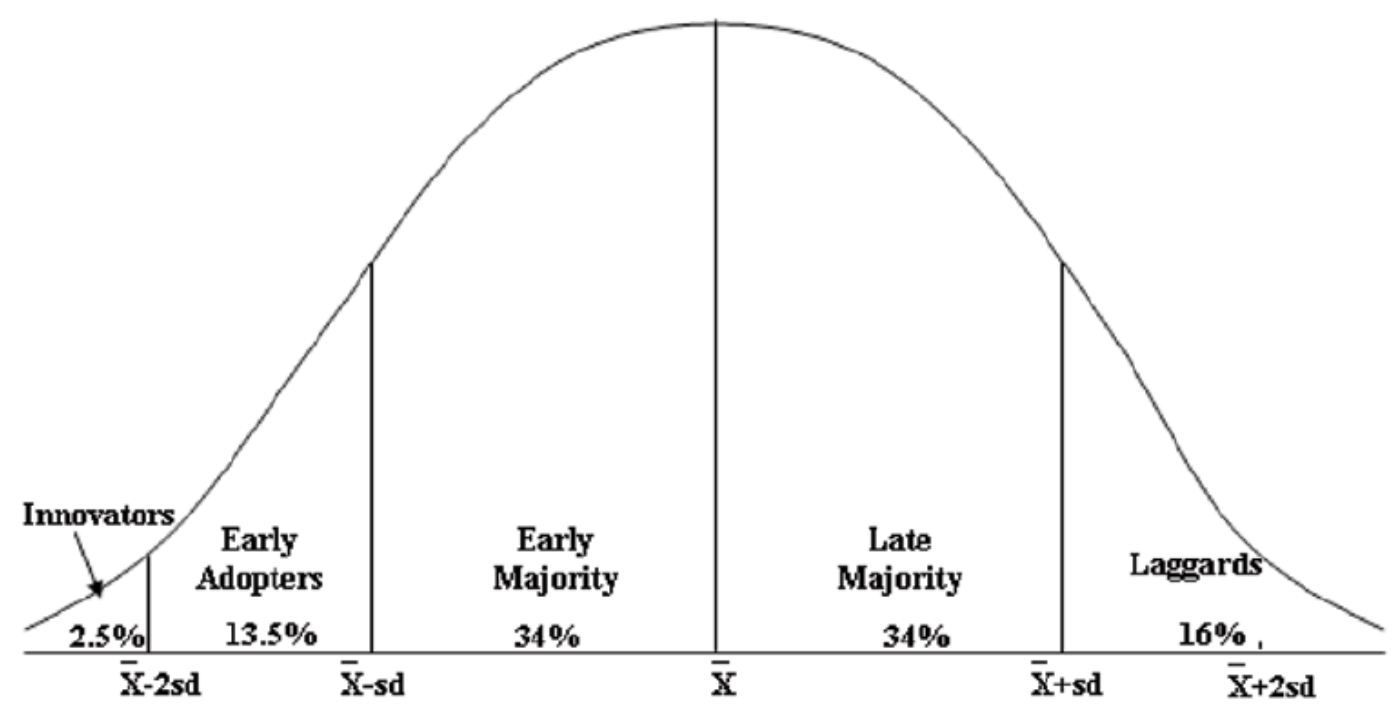

Figure 2.1 Rogers' (2003) Adopter Categorization on the Basis of Innovation 
Once an innovation has been introduced to a social system, diffusion begins. Rate of adoption, which is the relative speed at which individuals of a social system adopt an innovation, can be determined by examining the five attributes of an innovation outlined by Rogers (2003): relative advantage, compatibility, complexity, trialability, and observability.

Relative advantage is "the degree to which an innovation is perceived as being better than the idea it supersedes" (Rogers, 2003, p. 229). Compatibility is "the degree to which an innovation is perceived as consistent with the existing values of past experiences, and needs of potential adopters" (Rogers, 2003, p. 240). Complexity is "the degree to which an innovation is perceived as relatively difficult to understand and use" (Rogers, 2003, p. 257). Trialability is "the degree to which an innovation may be experimented with on limited basis" (Rogers, 2003, p. 258). Observability is "the degree to which the results of an innovation are visible to others" (Rogers, 2003, p. 258).

The innovation-decision process is "the process though which an individual (or other decision making unit) passes from gaining initial knowledge of an innovation, to forming an attitude toward the innovation, to making a decision to adopt or reject, to implementation of the new idea, and to confirmation of this decision" (Rogers, 2003, p. 168). This model is shown in Figure 2.2. 


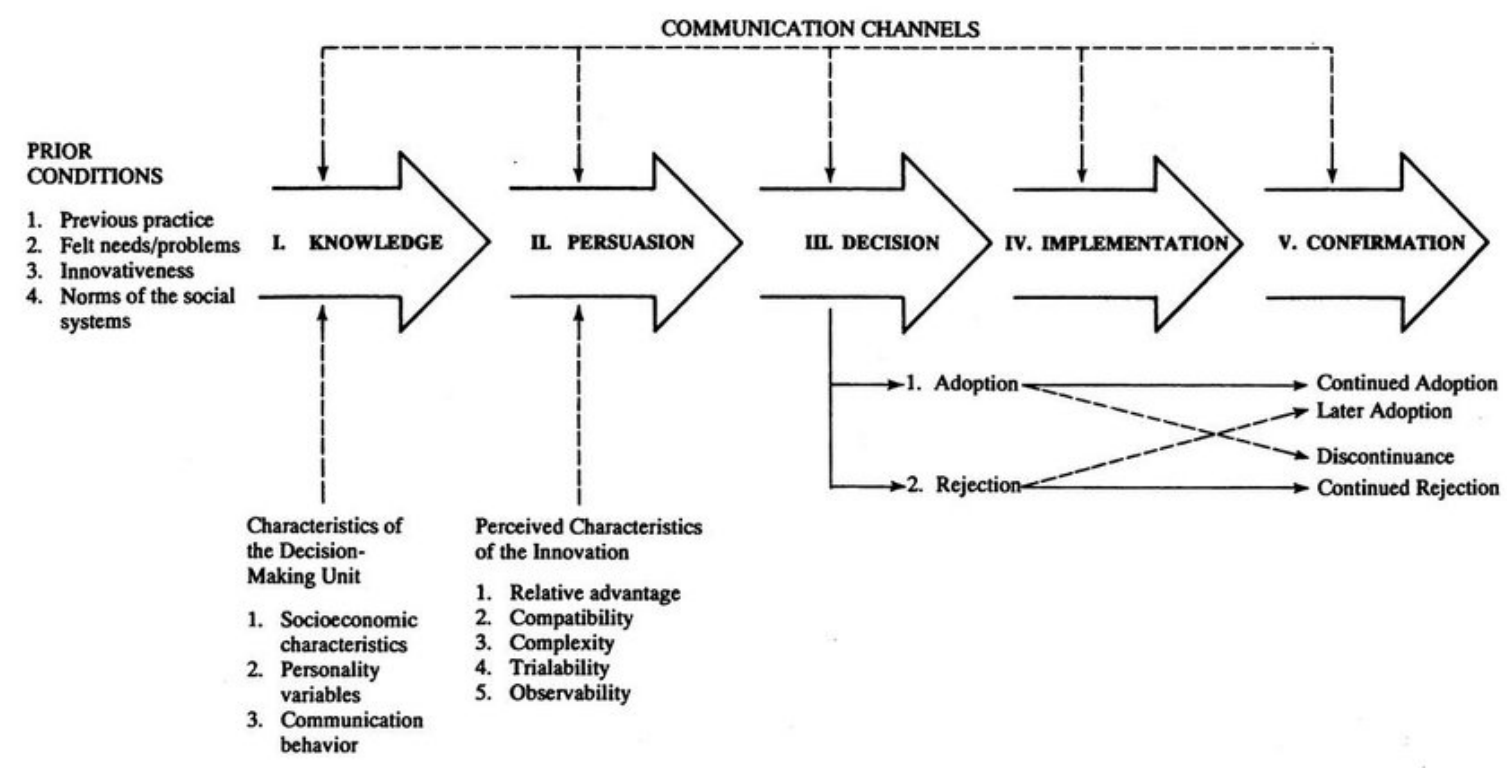

Figure 2.2 Rogers' (2003) Model of Five Stages in the Innovation-Decision Process

An individual's decision to adopt an innovation is not instantaneous; it is a series of actions that occur overtime (Rogers, 2002, p. 169). The nature of this process is represented through five stages. Knowledge "commences when an individual is exposed to an innovation's existence and gains an understanding of how it functions" (Rogers, 2003, p. 171). Persuasion is when "the individual forms a favorable or unfavorable attitude toward the innovation" (Rogers, 2003, p. 174). Decision takes place "when an individual engages in activities that lead to a choice to adopt or reject an innovation" (Rogers, 2003, p. 177). Implementation occurs "when an individual puts an innovation to use' (Rogers, 2003, p. 179). Lastly, confirmation takes place "when an individual seeks reinforcement for the innovation-decision already made" (Rogers, 2003, p. 189). 


\section{CHAPTER III}

\section{Methodology}

\section{Purpose}

The Extension service plays a vital role in today's society by providing communities with valuable information. The national pandemic of COVID-19 shifted how the Extension service was able to network with community members. Due to these unforeseen circumstances and protocol established to keep individuals and communities safe, the Extension service began developing new ways to maintain connection with the public. The purpose of this study was to explore and describe how WVU Extension Service is utilizing innovative social media practices to remain connected with clientele as a response to protocol enacted by executive orders to slow the spread of COVID-19.

\section{Protocol}

Interviews were conducted to collect qualitative data. A semi-structured interview was used to gather information. This method incorporates both structured questions and natural conversation, which allows the interviewer to maintain structure while exploring in depth concepts. The structured questions were developed to produce thought provoking answers from the interviewees. Follow-up questions were utilized to gain additional insight on valuable information shared by the participants. The purpose of the interview questions was to gather information needed to fulfill the purpose and objectives of this study. 


\section{Guiding Questions}

1. Can you describe what you would consider to be beneficial social media practices that you implement for work purposes?

2. When was your clientele most engaged with your social media posts and how could you tell?

3. What are some key examples of times you utilized social media to remain connected with clientele as a response to COVID-19 protocol?

4. What was something you thought would go well but didn't, and why?

5. Your survey response indicates that you used these (Facebook, Padlet, Instagram, Twitter, Snapchat, YouTube) social media platforms to remain connected with clientele.

a. Provide an example of an innovative way you used each platform.

b. Describe how you used different platforms for different purposes.

6. Social media allows Extension to reach new audiences, potentially individuals who are unfamiliar with Extension.

a. Can you share an experience that supports or denies this statement?

b. Has this become predominantly more true during COVID-19? Why?

7. What do you hope your clientele gains through your efforts of utilizing social media, and why?

8. How is social media usage for work purposes changing and how can you adapt to this?

9. Describe any challenges you foresee needing to overcome in order to continue utilizing social media practices in the future?

10. Describe how you work within guidelines for using social media platforms. 
11. Do you consider yourself an innovator when in regards to social media practices and why?

12. Can you describe a time you noticed a WVU Extension agent (other than yourself) utilizing innovative social media practices that you found beneficial.

13. Do you have any additional information pertaining to my research you'd like to add or any questions for me?

\section{Population}

The target population for this study was West Virginia Extension service agents who utilize innovative social media practices to remain connected with clientele as a response to protocol enacted by executive orders to slow the spread of COVID-19. Agents in all fifty-five counties in West Virginia were considered. In West Virginia, there are three main program units in Extension: Agriculture and Natural Resources, Family and Community Development, and 4-H Youth Development. According to the WVU Extension Service Directory (2020), there are 102 Extension agents and 6 agents in training employed by WVU Extension Service as of July 20th. However, some individuals serve as Extension agents for multiple counties and/or multiple program units. Therefore, 100 Extension agents and agents in training in West Virginia were considered for the target population. The potential participants were narrowed down and four agents were interviewed.

\section{Research Design}

Examination of the target population was completed utilizing qualitative methods. This data was collected through interviews. Guiding questions were developed with the purpose of 
gathering information that reflected the purpose and objectives of the study. Participants were selected based on results from a survey designed to identify West Virginia Extension agents who utilize innovative social media practices as a response to protocol enacted by executive orders to slow the spread of COVID-19. Additionally, the target population list was verified and other recommendations were given by the Director of Marketing and Communications for WVU Extension Service.

Interviews were conducted via Zoom during a three week period. These interviews were recorded via Zoom to ensure accuracy during the transcription and analysis process. Transcriptions were completed by the researcher.

\section{Selection of Participants}

An online survey was administered via email to all 100 Extension agents and agents in training in West Virginia. Administration of the survey closely followed guidelines and recommendations of Dillman (2014). Survey participants received a notice of the survey via email on the Friday prior to receiving the survey. The survey was emailed to participants at 9am on a Tuesday and included a cover letter containing detailed information pertaining to the survey and research. A first follow-up email was sent via email one week after the survey distribution. The second follow-up and final reminder email was sent the following Monday and reminded participants that responses need to be completed by the end of the week. The reminder emails were not identical to keep the participants attention and increase legitimacy of the survey (Sauermann \& Roach, 2013). On the Friday of the third week of the survey being open, the survey closed. 
Since the main purpose of the survey was to identify individuals for interviews, the survey utilized filter questions at the beginning. If individuals did not meet the criterion by selecting "no" to the filter questions, they were redirected to the demographics portion at the end. If individuals met the initial criterion, they continued to the body of the survey before the demographics section. The body of the survey consisted of ordinal questions relating to social media usage. Higher responses on these body questions indicate a higher use of innovative social media practices. The demographics section of the survey included only relevant information for contacting participants in the future for interviews.

Two pilot studies were administered to Extension agents in surrounding states, including Pennsylvania and Ohio, as well as various professionals in the Extension field. Any necessary changes to increase reliability were made before the administration of the survey to the population.

Survey results were examined by the researcher. Higher response rates on the ordinal questions indicated a higher level of innovativeness on social media practices for work purposes. Participants who had the highest response rates on the body of the survey were compared and narrowed down to four individuals. These individuals were confirmed by a professional in the industry. Additionally, one individual was added to the target population by recommendation from a professional in the industry. Overall, a total of five individuals were contacted for participation in the interviews.

\section{Data Collection}

The five extension agents were contacted via email on November 19, 2020. Attached to the email was the link to sign up for interview times. The interview slots were each an hour long 
from $8 \mathrm{am}$ to $5 \mathrm{pm}$ for a three week period beginning on December 1, 2020. Follow-up emails were sent to those who did not respond the following week. Interviews were scheduled and completed with four of the five selected individuals during the three week period. The interviews were held via Zoom and were approximately one hour in length. Holding interviews through Zoom was convenient for interviewees because it allowed them to participate at a time and place of their choosing. All interviews were recorded with interviewee permission and transcribed by the researcher.

\section{Research Rigor}

Trustworthiness of qualitative research methods was established through Lincoln and Guba (1985). Their model has four main criterion to meet in order to establish trustworthiness: (1) credibility, (2) transferability, (3) dependability, and (4) confirmability. Credibility establishes confidence that the findings are true. Transferability establishes that the findings can be applied in other instances. Dependability demonstrates consistency and replicability in the findings. Confirmability establishes that the findings were formed by the participants and not researcher bias. (Lincoln \& Guba, 1985).

For this study, triangulation and member check was used to establish credibility. Triangulation was completed by providing multiple sources of data, which included the video recording, audio recording, interview notes, transcribed data, and transcribed notes. Member check was completed by discussing interpretation of the data with committee members during analysis. Thick descriptive data was collected during this research to establish transferability. Dependability was established by providing detailed methodology to assure the research could be repeated and produce the same results. Triangulation was also used to establish confirmability. 


\section{CHAPTER IV}

\section{Outcomes}

\section{Introduction}

Outlined in this chapter are the outcomes from the interviews with four West Virginia Extension agents. These participants were selected based on survey results administered to identify Extension agents in West Virginia utilizing innovative social media practices as a response to COVID-19. The purpose of this study was to explore and describe how West Virginia University Extension Service is utilizing innovative social media practices to remain connected with clientele as a response to protocol enacted by executive orders to slow the spread of COVID-19. The following objectives were developed in order to achieve the purpose of this study:

1. Describe innovative social media platforms being used.

2. Describe how innovative social media practices are being used.

3. Describe why innovative social media practices are being used.

4. Identity perceptions of barriers and needs related to utilizing innovative social media practices.

A total of 52 transcribed pages from the four interviews were utilized for data analysis. These pages were transcribed and interpreted by the researcher. 


\section{Participants}

Outlined in Chapter III, the target population for this study was West Virginia Extension service agents who utilize innovative social media practices to remain connected with clientele as a response to protocol enacted by executive orders to slow the spread of COVID-19. These participants were selected based on results from a survey created for the purpose of identifying these participants. Survey results for the participants are outlined below in Table 4.1, Table 4.2, and Table 4.3. The demographics pertaining to participants' social media usage as a response to COVID-19 are included for the purpose of building each participant's persona. For the purpose of this study, participants have been given pseudonyms.

\section{Table 4.1}

Participant Program Unit Information

\begin{tabular}{lccc}
\hline Pseudonym & \multicolumn{3}{c}{ Agent Program Unit } \\
\cline { 2 - 4 } & Agriculture & 4-H & $\begin{array}{c}\text { Family \& Community } \\
\text { Development }\end{array}$ \\
\hline Sarah & Yes & Yes & Yes \\
Elizabeth & No & Yes & Yes \\
James & No & Yes & No \\
Kimberly & Yes & Yes & Yes \\
\hline
\end{tabular}

Note: $\mathrm{N}=4$ 


\section{Table 4.2}

Social Media Platform Usage by Participant

\begin{tabular}{lcccc}
\hline Pseudonym & \multicolumn{3}{c}{ Social Media Platform } \\
\cline { 2 - 5 } & Facebook & Instagram & Padlet & YouTube \\
\hline Sarah & Yes & Yes & Yes & No \\
Elizabeth & Yes & Yes & Yes & Yes \\
James & Yes & No & Yes & Yes \\
Kimberly & Yes & No & Yes & Yes \\
\hline
\end{tabular}

Note: $\mathrm{N}=4$

Table 4.3

Participant Social Media Usage As a Response to COVID-19

\begin{tabular}{lcccc}
\hline Pseudonym & Increase SM & Held virtual & Community & Communicate with \\
& usage & Activities on SM & engagement on SM & community on SM \\
\hline Sarah & A lot & A moderate amount & A little & A lot \\
Elizabeth & A great deal & A great deal & A lot & A great deal \\
James & A great deal & A great deal & A great deal & A great deal \\
Kimberly & A great deal & A great deal & A great deal & A great deal \\
\hline Note: $\mathrm{N}=4 ; \mathrm{SM}=$ Social Media & & &
\end{tabular}

Note: $\mathrm{N}=4 ; \mathrm{SM}=$ Social Media 


\section{Sarah}

Sarah is an Extension agent who covers all three program units for her county. Survey results indicated that she utilizes Facebook, Instagram, and Padlet for work purposes. She indicated that she has increased social media "a lot" as a response to COVID-19 as well as communicates with community members "a lot" through social media platforms.

\section{Elizabeth}

Elizabeth is a 4-H and Family \& Community Development agent for her county. Her survey results indicated that she utilizes Facebook, Instagram, Padlet, and YouTube for work purposes. She also indicated that she has increased social media usage and held virtual activities "a great deal" as a response to COVID-19. Additionally, she indicated that she communicates with community members "a great deal" through social media platforms.

\section{James}

James is a 4-H agent who indicated that he uses Facebook, Padlet, and YouTube for work purposes. His survey results indicated the highest responses in: increased social media usage, virtual activities held, community engagement, and communication with community members through social media platforms.

\section{Kimberly}

Kimberly is the agent for all three program units in her county. She indicated on her survey that she utilized Facebook, Padlet, and YouTube. She also responded the highest in: 
increased social media usage, virtual activities held, community engagement, and communication with community members through social media platforms.

\section{Research Objective 1}

Identifying the innovative social media platforms being used by West Virginia Extension as a response to protocol enacted by executive orders to slow the spread of COVID-19, was the first objective desired to learn by the researcher. The researcher developed questions to gather the information needed to meet this objective. The researcher analyzed the data by recognizing every time the interviewee mentioned utilizing an innovative social media platform to remain connected with clientele as a response to COVID-19.

A word cloud, shown in Figure 4.1 was developed to reflect what social media platforms are being used by West Virginia Extension and how often they were discussed in the interviews collectively. Each platform discussed can appear once per interview. This means that each platform can appear up to four times. For example, if Facebook was referenced in every interview, then the word Facebook will appear four times. The size of the word indicates how many times the social media platform was discussed by the interviewee in regards to utilizing the platform to remain connected with clientele as a response to COVID-19. 
Figure 4.1

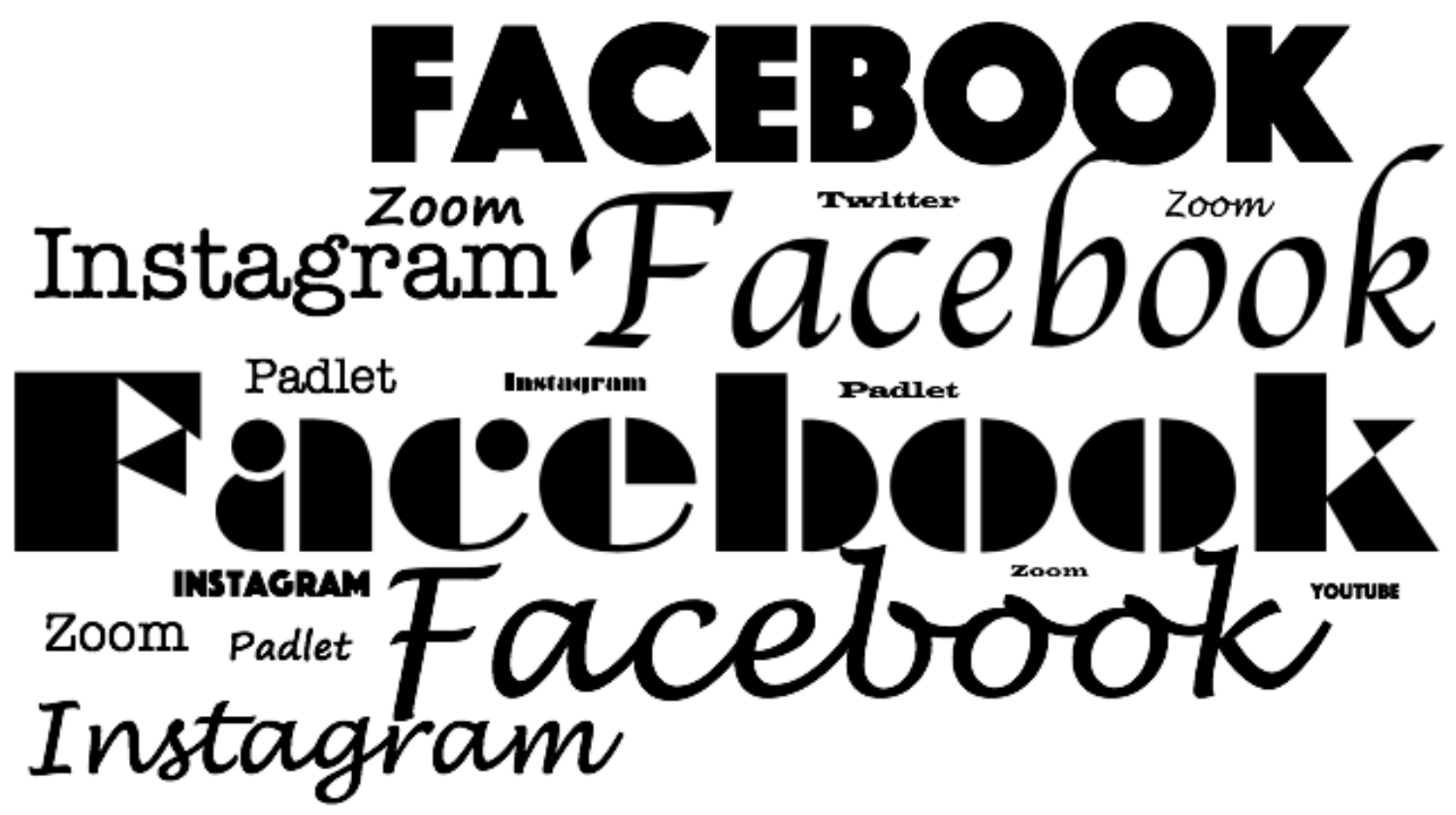

There was a total of six innovative social media platforms being utilized by WV

Extension to remain connected to clientele as a response to COVID-19. The most commonly used platform being Facebook, followed by Instagram, Zoom, and Padlet. Social media platforms such as Twitter and YouTube are also being used.

The researcher analyzed the transcribed data through cross-case analysis. A visual providing open and axial coding for how various social media platforms are being used is provided in Table 4.4 . 
Table 4.4

Coding for How WV Extension is Using Innovative SM Platforms As a Response to COVID-19

\begin{tabular}{|c|c|}
\hline OPEN & AXIAL \\
\hline Facebook & $\begin{array}{l}\text { - } \text { Virtual 4-H Camp } \\
\text { - } \text { Club Pages } \\
\text { - Virtual Activities, Programs, \& Events } \\
\text { - Community Development } \\
\text { - } \text { Mostly reaching adults/parents } \\
\text { - } \text { Recruitment }\end{array}$ \\
\hline Instagram & $\begin{array}{ll}\text { - } & \text { Photos } \\
\text { - } & \text { Stories } \\
\text { - } & \text { Mostly reaching youth }\end{array}$ \\
\hline Padlet & $\begin{array}{ll}\text { - } & \text { 4-H Camp } \\
\text { - } & \text { Meetings } \\
\text { - } & \text { Safe }\end{array}$ \\
\hline Zoom & $\begin{array}{l}\text { - Virtual meetings } \\
\text { - } \quad \text { 4-H Camp }\end{array}$ \\
\hline Twitter & - Personal account \\
\hline YouTube & - Video links \\
\hline
\end{tabular}

Note: $\mathrm{SM}=$ Social Media

\section{Facebook}

The most commonly used platform discussed was Facebook. Interviewees shared that Facebook is a great way to share a wide variety of information for work purposes. Facebook is also a very commonly used platform for people of all ages, therefore, it does a successful job at 
reaching the intended audiences. Elizabeth referred to Facebook as her "catch all" in regards to distributing information. She stated:

Facebook is just kind of my catch all. (...) I tried to push, push most of our information out on Facebook (...) a larger crowd of people on Facebook. I felt on Facebook, we can reach the grandparents, we can still reach the kids for some of the kids that are still using Facebook. Every generation is kind of on there. We always try to provide something for each of them. And it's more of a recruitment tool, I feel like than Instagram.

Although Facebook is a great way to shared information and keep clientele up-to-date, Kimberly noted the that these Facebook posts are used in conjunction with other means of communication, such as postcards and emails. These updates are also a great way to showcase 4Hers and other members in the community. When asked how she uses Facebook to remain connected with clientele, Kimberly stated:

Facebook is Facebook. We use it for all of our updates, any information that we're going to send out on like a postcard mailing, we try to post on Facebook also. And then we use that for camp programs and our livestock stuff. (...) Any of our updates, anytime anyone like has an award or an accomplishment or newspaper article, we link all that into our Facebook.

Kimberly also mentioned:

I think about when I share a Facebook post too because it could be a Facebook post that like I just thought was interesting or I knew other people needed the information on, I 
would instantly share it. But if that came in an email, I wouldn't forward it to all of my email contacts.

When discussing Facebook usage with 4-H agents, many mentioned that Facebook is an effective way to reach parents. The fact that agents are finding the audience on Facebook to be predominately 4-H parents and families, should be taken into consideration when creating and developing posts. Sarah mentioned:

Facebook is how I communicate when I want adults and parents to know something that's going on. (...) I keep that in mind when I do a post on Facebook who the audience is, who's seeing it. Generally it's 4-H families that I'm pushing that information to.

Although agents may want to target the 4-H members on Facebook, agents recognize that they are typically reaching their parents. Kimberly stated: "We have been on Facebook primarily as far as outreach goes and I know that we target our kids through social media, but the truth is, on Facebook I think we're getting a lot of their parents."

For 4-H specifically, Facebook groups and pages are used by clubs. Although these groups and pages may be created and managed by club leaders, Extension agents have access to these groups to share information and communicate with club leaders, parents, and members. Sarah discussed: "Most of our [4-H] clubs have their own group or page on Facebook so they can plan events or just talk amongst themselves." 
Facebook was also utilized as a host for virtual 4-H Camp since in-person camp was not an option in this county at the time. Most components of traditional in-person camp were still incorporated as best as possible. The goal of virtual camp was to make it as similar as possible to the traditional in-person camp that 4-Hers know and love. Materials for camp classes and other activities were mailed to campers. Campers would complete various activities at home and post about them on Facebook. They could communicate with other campers through this platform as well. James stated:

So, Tri County camp, kind of got flipped on the end. (...) So we did Tri County virtual fundings. And essentially what we did was we sent out a complete box of activities with camp classes, assemblies, crafts, a little campfire pin, like all kinds of stuff that would remind the kids of camp or make it as camp esque as possible. And then they actually would $\log$ in for specific times. (...) We had 238 Kids sign up for it. And we had I believe right around 200 (...) that were engaged every day. (...) Every day we had some type of challenge, every day they would post pictures (...) For all of that we actually used Facebook.

A private Facebook page was utilized to ensure that only those registered for 4-H camp had access to the page. When asked if he implemented a private Facebook page that anybody that was registered for camp would be able to access, James responded: "Yeah. So we sent a private link once they registered."

Beyond 4-H, Facebook is utilized for community development activities and programs. West Virginia Extension agents have conducted various programs as a response to COVID-19. 
Some of these efforts that stand out involve making sure families had their necessities after the shutdown due to COVID-19. Elizabeth discussed her efforts in doing this:

This past summer we did a foods program for families in our county (...) and the information came out for this right after all the school shut down. So I didn't have any way to get information out to families through this whole system (...) But I would say $90 \%$ of my responses came from a Facebook post asking if people will be interested in a box. And that's, we got a lot of outreach from that, that it was folks that we normally wouldn't have reached before. And then this program was derived from, you know, reaching families during covid and making sure they had their necessities met.

James discussed how he utilized Facebook to promote various programs as well. $\mathrm{He}$ stated:

I call them one and done programs. (...) But it hasn't just been kids, we also do teacher buttons, where teachers from [West Virginia] County, or any staff I should say that employed by the Board of Education, could request a button that way they could wear it with their mask. And we only send it out by Facebook. We didn't send it out any other way. (...) So we put it out, and it probably had every bit of 1200 likes, shares, you know, reactions. And we ended up making over 300 buttons.

James also mentioned:

We've had a couple of different kind of collections. We've done a food collection. We've done- We're actually just now doing a toy collection. We've done school collections, 
we've done all kinds of stuff. And I think the ability for us to get that information out has been huge.

\section{Instagram}

Another common social media platform, Instagram, is used by 4-H agents to target members more than parents. Targeting a younger audience also means altering how they post. Aspects such as language, tone, etc. changes to meet the needs of the younger audience. Although the platform is for all ages, agents notice that the kids are typically the ones seeing the posts. Instagram stories are also utilized to engage with younger 4-H members. Elizabeth mentioned:

If I'm targeting younger kids on Instagram, I'll probably try to put stuff on like the Instagram stories. (...) The older generation doesn't know how to use Instagram stories and that type of stuff. But it's kind of an all age type platform to use.

Since mostly 4-H members themselves are seeing the posts on Instagram, engagement in posts seems to increase when more pictures are posted with familiar faces. Additionally, the poll feature on Instagram stories is a quick way to engage with members. Sarah stated:

I think our Instagram tends to get about the same likes per post. It doesn't seem to drastically change. (...) The ones that I know that get a lot more. Well I've noticed that they are um like collage posts seem to get a lot more. And I don't know if it's because they see more kids' faces in those, especially after camp or a large event where I share like multiple photos at once. Those seem to get a lot of feedback. And I've been using 
stories on Instagram more and more and anytime I ask a question, like one of those poll questions, I see 4-Hers respond to those.

\section{Padlet}

A newer social media platform that Extension agents in the state of West Virginia are beginning to familiarize themselves with is Padlet. This platform is used to share information with 4-H members. Agents expressed that although it is newer and may not have as much engagement as other platforms, they believe it to be safer for members because the chances of outsider access is very slim compared to similar platforms, such as Facebook. Kimberly discussed:

As far as Padlet goes, it's just a baby in this county at this point. It's more of a bulletin board where we just try and get some information out there so that people have access to it but I don't find it to be very interactive at this point- more of just like a kind of a storage space for information that kids could access. (...) I do like that it's safer than using Facebook because we can isolate it and, you know, not have to make a private Facebook page. So it's a private Padlet page that always stays there.

Sarah mentioned that Padlet is being used in place of in-person meetings. She said: "[Padlet's] kind of a tool that we are using in place of in person meetings this year."

Padlet was also used as a platform to conduct virtual 4-H camp. Although the engagement was found to be much lower than Facebook, it provided a great alternative for those who did not have access to Facebook. When discussing conducting virtual 4-H Camp through 
predominantly Facebook, James stated: "We did also have Padlet, but we did not have very many kids actually engage in Padlet. (...) So we didn't really know how to incorporate it as best we could."

When asked if he had difficulties where a camper didn't have any way to access Facebook for the virtual 4-H Camp group, James responded: "That's why we had the initial Padlet setup. We were very fortunate that we could offer them through email or Padlet. I think there were only 5 out of 238 that did not have Facebook."

\begin{abstract}
Zoom
Zoom is a social media platform that has taken off as a response to COVID-19. In person meetings used to take place regularly in Extension. Since this has been limited recently, Zoom has provided a great alternative for West Virginia Extension agents. 4-H club meetings would typically happen in-person every month, but are now taking place through Zoom. Although participation isn't as high as in-person meetings, agents are still discussing a decent amount of participation. Sarah mentioned: "And that's kind of taking the place of club meetings because we aren't able to meet in person. (...) Anyone can get on Zoom no matter where you are.”
\end{abstract}

Kimberly also discussed:

So we've done some county wide and club specific 4-H Zoom meetings. So what would be a normal monthly meeting, we've been able to host it on Zoom. And we've had about $50 \%$ participation, which for normal club meetings we usually get about $75 \%$ of participants- or participation. So I feel like $50 \%$ is pretty good. So we've been able to use 
that for club meetings, county and club, or like county wide and just local clubs. And we've done it for our 4-H leaders.

When discussing virtual 4-H Camp, James referenced how zoom calls were utilized by noting: "That included Zooms, which included some cabin meetings and council circle."

\section{Twitter}

Although Extension agents do not have their county accounts on Twitter, some agents have personal accounts. These accounts still remain professional and can be used to share information posted by organizations and pages within the agricultural industry. Elizabeth discussed how she uses her personal Twitter by mentioning:

I follow the Extension page, WVU Extension Twitter page. (...) If our Dean put something out on there I'll like it or I'll retweet it. I usually don't put out any recruitment stuff on Twitter just because I don't have a lot of kids on my Twitter- my personal Twitter. It's a private account. (...) I'll share just basic stuff on there.

\section{YouTube}

Although people are not directly following Extension YouTube accounts, Extension agents upload videos through YouTube and share the links. YouTube links can be attached to emails as well as social media posts for easy access. Kimberly stated:

So we've used YouTube for video links. When we did our county livestock show and sale you could see some videos there. We also, with camp in a box, had some video links to like a frog hunt so we had a going deeper video, you know, you could do the frog hunt 
with just the paper that supported it or you could go deeper and watch the video link that was available on YouTube.

\section{Research Objective 2}

The second research objective was to describe how innovative social media practices are being used by West Virginia Extension as a response to protocol enacted by executive orders to slow the spread of COVID-19. The researcher analyzed the transcribed data through cross-case analysis. Methods for West Virginia Extension utilizing innovative social media practices were determined through the use of open coding. A visual providing axial coding in relation to what to post and when to post is provided in Table 4.5.

Table 4.5

Coding for How WV Extension is Using Innovative SM Practices As a Response to COVID-19

OPEN

AXIAL

What To Post

- Photos

- Incentives

- Brief content

- Intentionality

- Time frames

When To Post

- Increase Usage

- Consistency

- Knowing clientele

Note: $\mathrm{SM}=$ Social Media 


\section{What to Post}

Extension agents found that being intentional and deliberate with what they post on social media has shown positive results. When face-to-face communication was still possible, Extension agents implemented social media, but not to the extent they are as a response to COVID-19. Putting more thought into what pictures to post, what information to share, etc. has resulted in an increased involvement on social media. When asked what has changed or been done specifically with social media posts from before COVID-19 compared to presently, Elizabeth shared:

Maybe just the way we approach - the way we post information. I know a lot of times before, you would just throw up a picture and you think people know what you're talking about. Now we add a little bit more of an explanation of what's going on, how to contact people, what's the best way to get the information to the extension office. And just adding more information and giving people more detail, I feel like gets them more involved as well.

Extension agents have discovered that photos receive a lot of attention on social media. Sharing photos catch the attention of their clientele, but also receive the most feedback. Clientele enjoy seeing familiar faces shared by West Virginia Extension agents. This is also an effective way to showcase and recognize community members, 4-Hers, etc. for their hard work and dedication to Extension. When discussing social media posts that receive the most feedback, Sarah mentioned: "The posts that get the most likes have photos (...) photos that are of people they know. So it's either volunteers of kids, especially if they are getting any kind of recognition for something." 
If Extension is hosting an online event or activity, Extension agents will share pictures of well-known community members participating in the activity or event Extension is promoting. Agents expressed that they found showcasing others participating while the activity is currently happening, increases the likelihood of others to participate as well. Kimberly discussed:

The more photos and connections to actual people that our county knows, the more enthusiasm we see behind stuff and a willingness to give it a try attitude. (...) They want to see the smiling faces and anytime that we can get a younger member to share a photo or a video or like an actual 4-Her versus a community project kind of thing. If we get those like high school age 4-H kids to do something, it seems pretty instant that our kids get excited about mimicking their adult peers.

West Virginia Extension agents are utilizing incentives as a way to increase involvement and engagement with their clientele. Offering incentives is an exciting method in promoting what Extension has to offer. James stated: "We found that a lot of times through social media we try to offer them some type of prize or some type of incentive."

When asked to share an example of a type of post that increases clientele engagement, Kimberly mentioned: "So offering incentives for things is exciting."

Social media is being used as a way to promote events and activities that are being hosted through Extension. Although these events are being shared on social media, only certain details of the information are included in the initial post. Certain aspects, such as Zoom links, are not 
being shared on social media to prevent safety issues. However, social media posts will include information of where to access the link. Sarah discussed: "Whenever we promote it, we say 'here's when it's happening. It's on Zoom. If you're a registered 4-Her, you will get an email with the link. (...) 'Be sure to check Padlet or check your email.”

Extension agents are keeping information brief and to the point when posting on social media. Posts on social media provide clientele with enough information to get a basic understanding of what is being promoted. Adding a note with the post about where to find additional information is also important in creation of promotional posts. James mentioned: "We tend to be very short and brief (...) and we always put 'for more information please check our website."

Providing time frames for clientele in regards to activities on social media has shown an increase in involvement. Extension agents expressed that if they leave things open ended, they see a decrease in participation. When sharing activities on social media, Extension agents provide deadlines that are not too far into the future to produce increased engagement. Kimberly discussed:

Offering things that have specific time limits on them seem to have better participation. So when we did our camp in a box camp (...) We said like, you have to do this by seven o'clock today. If we put deadlines on things, our participation increased. When you leave things open ended it seems like people are like, 'oh yeah I'll get back to that' or 'I'll do that' and then they forget about it. Or they lose interest. But when things are like real time and interactive, we had better participation. 


\section{When to Post}

Extension agents are utilizing social media as a response to face-to-face communication failing. With an increase of social media, agents are seeing an increase in engagement and feedback as well. Kimberly discussed:

Since covid we've more than doubled our communication on Facebook, when I went to pull the reports this year for my faculty file, we've had, I think it was like 100,000 more contacts than the year before. (...) I know we've been innovative because we've been able to reach so many people, and those numbers, you know, they're not perfect but they do definitely tell a story of how many people had a connection to (..) county 4-H. That wouldn't have had that same connection otherwise.

Elizabeth also mentioned: "Our Extension page did have an increase in likes over covid times."

Consistency is important in implementing social media for Extension. If a specific event or activity gets a lot of attention and positive feedback, agents would do it again. When this leads to becoming a regular thing, whether it's weekly, monthly, etc., feedback in engagement increases. Sarah discussed a time when social media engagement was high due to regularity of the posts. She said: 
It got such good response that really I did encourage her to like, 'oh, you should do it again this week!' And then it just really became her thing. Every week she would go live right at the beginning of the market.

Knowing when your clientele can be most engaged in what you are posting, is an important aspect for Extension agents to consider. If an activity is being posted for young 4-Hers who need parent supervision, one agent found that posting in the evening, between 6pm and 8pm, allows parents to help their children with the activity. Kimberly discussed:

As far as what time, If it's something that requires parent help, so our younger kids who don't have social media accounts, it's better to do it in the evening after people have been home for a little bit, had dinner. We aim for like that six to eight o'clock window.

Looking at Facebook analytics to see what times posts get the most attention is a valuable tool for Extension agents. One Extension agent used this and found that his clientele were most engaged with posts during lunch hours, between $11 \mathrm{am}$ and $1 \mathrm{pm}$. These analytics were viewed prior to COVID-19, but have been utilized more during COVID-19 with the increase of social media usage. Not all posts will be sent out during the same window of time. Another viable option for sharing posts is late at night so that it is viewed first thing in the morning. James shared:

So we've actually looked at this as far as I mean Facebook specific. You can go in and see when you get the most hits. So we always try to get all of our published stuff out right around 10:30, because we found that most working adults check their phone somewhere between the hours of 11 and 1 . And that's when they'll see most notifications as well as 
emails. (...) We started to notice it early on at the end of 2019, and then for 2020 it really started taking effect. We started sending a lot more stuff out, you know, not necessarily in the evenings. So we either aim for that 10 .. We like to post at 10:30 so we get that 11 to 1 , or we'll wait and actually post it after $10 \mathrm{pm}$, because then it shows up in their morning cycle as they're going through and getting ready.

Recognizing which days of the week clientele are more likely to be engaged is important as well. Extension agents may often work on weekends when they are hosting specific activities and events. However, agents have found that having the majority of activities during the typical work week, has been successful. Having weekends open allows clientele time to focus on other aspects. Kimberly mentioned:

I don't try to do a whole lot on the weekends because I feel like everybody's trying to take a breath. So we were trying to keep it in like that Monday through Friday, unless it was something where we could be, you know, real time interactive with them. We kind of stray away from the weekend activity.

\section{Research Objective 3}

The third research objective was to describe why innovative social media practices are being used by West Virginia Extension as a response to protocol enacted by executive orders to slow the spread of COVID-19. The researcher analyzed the transcribed data through constant comparative analysis. Communication, accessibility, promotion, and engagement were identified as four main reasons why innovative social media practices were being used by West Virginia 
Extension through the use of open coding. This visual representation of open and axial coding is shown in Table 4.6.

Table 4.6

Coding for Why WV Extension is Using Innovative SM Practices As a Response to COVID-19

OPEN

AXIAL

Communication

- Accuracy of content

- Promptness

- Efficiency

Accessibility

- No geographical restriction

- Timely

Promotion

- Reach new audiences

- Recruitment

Engagement

- Maintain connection

Note: $\mathrm{SM}=$ Social Media

\section{Communication}

Extension agents are utilizing social media for communication. The general public is already using social media platforms in their personal lives, making it a successful way to share information with clientele. Other means of communication, such as post cards and emails are still being utilized, but social media is receiving the most reactions. James stated: 
I think the most beneficial is just keeping the general public up to date (...) We've sent just plain emails, we've sent postcards (...) but in a digital age that we're now in, social media tends to get the most publicity, as well as the most feedback.

Considering that social media has proven to be an effective way of sharing information, it's also been beneficial for cancelations. The past year has been untraditional as a response to COVID-19. Plans are everchanging in a short amount of time. Social media provides Extension agents with the ability to share information, such as cancelations, with clientele in a timely manner. Kimberly discussed how social media elicits "real-time communication." She stated: We've used it to get information out. (...) It's been a great. I mean it's a real-time way of communicating cancellations of things, which unfortunately this year we've had a lot of cancellations that we've needed to get out to people very quickly so we've used it for some real-time communication.

The decrease of time it takes to communicate and share necessary information with the public is worth noting. Social media is allowing agents to significantly cut down the time it takes to share information with their clientele. When discussing his thoughts on utilizing social media more in times on COVID-19, James mentioned:

But it has ultimately kind of been a blessing to us to be able to get that kind of information out, because we can do it. What used to be in a newsletter or postcard and take five days to get to them they're now getting in a matter of seconds. 
Another reason Extension agents are using social media is to better control the accuracy of the information being posted. When face-to-face communication was being utilized more with 4-H clubs, information was often shared through word of mouth. This made it more difficult to control the accuracy of the content. Since face-to-face communication has failed, social media has been utilized in its place. When discussing communicating with 4-H clubs, Sarah expressed how social media allows her to share exactly what she wants with club leaders and members, as well as assure all necessary information is included. She said: "If I'm the one posting it... then I know exactly what I'm saying and making sure all the details are there"

\section{Accessibility}

WVU Extension is also utilizing social media because there is no geographical restriction when providing clientele information. It allows agents to communicate with clientele from anywhere at any time. When discussing social media usage, Sarah mentioned: "A geographical restriction doesn't apply anymore (...) And what's been beneficial is that we can push out information at all hours of the day."

Because of the accessibility that social media provides for communication between Extension agents and clientele, time has been saved. Less time is being spent waiting for information to get from one individual to another. When asked the purpose of utilizing social media for posts, James responded: "I would say accessibility. (...) [Social media] has truly cut down the turnaround time to get information out." 
Kimberly also mentioned that social media fosters a faster turnaround of sharing information. She stated: "It gives us an opportunity to have things that we can post with a shorter turnaround."

\section{Promotion}

Seeing that community development is an important goal of Extension, agents use social media as a way to promote WVU Extension and what it has to offer for the community. When asked what she hopes clientele gain through her efforts utilizing social media, Elizabeth stated:

I feel like they just need to know more about what Extension can do for the communities. We try to post a wide variety of, you know, what our ag agent is doing and what the 4-H program is doing. But it's not just what 4-H is doing and what the ag agents doing, it's more of different services we can provide such as soil testing, (..) pressure canner testing, (...) how to get the kids involved. (...) They tell us all the time, we're the front door to WVU. (...) We're in every county. We're 55 counties across the state. I hope they, you know, gain some knowledge of what extension can provide for everyone in the county.

Social media is a great tool to help promote aspects of Extension, such as 4-H. Agents want community members to know what the $4-\mathrm{H}$ program is doing and providing for the community. Sarah stated: "I want the public to know what (...) County 4-H is doing. And so it is a tool to promote (...) what we are bringing to the community." 
Utilizing social media to promote Extension has allowed programs, such as 4-H to reach new audiences. Social media usage has been increasing since face-to-face communication failed, which also increases the amount of types of people being reached. This has been a great opportunity for recruitment within Extension. Kimberly discussed her increased usage of social media in relation to promotion. She said:

We've increased it [social media usage] and it has given us a vehicle to really connect to some people that we wouldn't have had the same opportunity to find without it. (...) I think gains community support and maybe a parent sees that and says 'oh my kid wanted to join 4-H' and 'how do I get them in there.' So it works more towards recruitment.

Elizabeth also discussed how the increase usage of social media helped her recruitment in 4-H specifically. She stated:

I got kids who aren't normally 4-H members to sign up. We had kids from my community that saw my post on Facebook, they followed the extension Facebook page, and they're like "oh that sounds really interesting, but I'm not in 4H.” I'm like, "That's fine." We actually had recruitment kind of go up this year because of folks seeing all of our stuff on social media, so that has been very helpful.

\section{Engagement}

WVU Extension is also using social media as a way to keep clientele engaged since faceto-face communication was no longer possible. The intentions for sharing information on social media is to maintain the engagement that Extension agents had with clientele prior to COVID- 
19. James expressed: "We just wanted to make sure that we were able to retain the level of engagement that we had had previously, but do it on that modified delivery kind of scope."

Prior to the pandemic of COVID-19, engagement in Extension was predominately taking place in person. With this no longer being possible, social media became a major factor in keeping community members and 4-H members engaged. Extension agents strived to find a way to maintain the engagement that they once had in person with their clientele. Sarah discussed:

Social media is a tool to get them [clientele] information and to keep them engaged (...) It's such a weird year, I just really want to keep kids connected to 4-H (...) Prior to the pandemic, we have had good support in our community. I just want to keep that at the forefront of everybody's mind.

\section{Research Objective 4}

The fourth research objective was established to identify perceptions of barriers and needs related to utilizing innovative social media practices. This objective was determined through cross-case analysis. Protections issues, separating work from personal life, accessibility, responsibility, and too much online were identified as perceptions of barriers and needs related to using innovative social media practices. The open and axial coding for this objective is outlined below in Table 4.7. 
Table 4.7

Coding for Perceptions of Barriers and Needs Related to Extension Using Innovative SM Practices As a Response to COVID-19

OPEN

AXIAL

Protection Issues

Separating Work \& Personal Life

Accessibility

Responsibility

Too Much Online
- Child Protection Issues

- ADA Compliant

- Safety

- Personal SM accounts

- Those without SM accounts

- Reaching clientele

- Who's responsibility is it to implement SM?

- Volunteers

- Clientele burnt out

- How to overcome this

Note: $\mathrm{SM}=$ Social Media

\section{Protection Issues}

Child protection issues is a barrier that Extension agents need to take into consideration when developing their posts. In order to share photos of 4-Hers, parents/guardians have to sign and return a media release form. When asked about potential barriers for social media usage in the future, Sarah responded:

We've talked about child protection issues. That's always something that's on our minds as far as I want to use images of kids because that's how we promote our program. And 
like I said, those are the popular posts. When they see their friends or when they see their grandkids, ya know, those posts get all the likes. (...) Kids who get shared by me on social media, they've all signed media releases. So like I have permission to do that.

Extension agents needing permission to post photos of 4-H members is a very important barrier to be aware of. Although there are a lot of parents that are excited for their children to be featured on social media, some parents do not want their children's picture out there. An additional challenge with this is the possible effects when a child realizes they are the only one not pictured. Depending on the age of the child, they may not understand why their friends are getting posted on social media and not them. This could unintentionally create division between children because they feel excluded or embarrassed. Kimberly shared her thoughts on this:

We have parents who are really uncomfortable with their children being featured on any kind of social media, which is understandable. The problem with it that I see is some parents are really excited to put their children on social media and see them, you know out there in the world. So it creates like, some kids are excluded from that. And when we talk about like awards and being proud of things and we've got everybody's photo but the one child who didn't get to be on social media. Those things kind of create division amongst our kids and embarrassment, you know, and whatever it is that the child who didn't get to participate feels. So I think that's a challenge for sure.

An unfortunate but realistic barrier of utilizing innovative social media practices is safety. Social media is a great way to promote events and increase community involvement. However, 
posting certain information for the public to view can potentially create a vulnerable situation. Kimberly provided an example of her concerns regarding safety:

Safety is going to be a challenge for us. I live in a rural place in a very safe place thankfully but advertising like 'we're going to have all the features at the Christmas parade.' Well, we just told all of social media that 200 youth, we're going to be at this specific place at this specific time. And that creates a really vulnerable situation. So, trying to have my eyes wide open and not be so like trusting of a platform that I've known since 2005 and realizing, to be very careful with those kinds of things as far as safety goes, and even you know as much as like our 4-H camp whenever we announced campaigns. I don't know if we should put them out there for the world to know versus in a private email a private mailing. So trying to you know differentiate between what's information that the world can know and what's information that could potentially make our kids more liable to a child abduction kind of thing, or, you know, numerous things that can happen this day and age.

Being compliant with Americans with Disabilities (ADA) is a current need when implementing innovative social media practices. Extension agents are mindful of this by including text in the body of the post and not just on pictures. Ensuring that all audiences are reached and included is an important aspect to remember. Elizabeth discussed being ADA compliant:

They preach to us all the time about being ADA compliant. And I know a lot of people still struggle with that, and it's because you have folks who use screen readers and stuff 
like that to read what's on their iPads or on their computers. So I would say that's probably the biggest challenge.

In relation to being ADA compliant, James stated: "With ADA you have to, of course, include all text that's on the picture in the body of the actual post."

\section{Separating Work from Personal Life}

A major benefit to utilizing social media is the fact that a large amount of people are already using it in their personal lives; this includes Extension agents. This provides community members, 4-H members and parents, etc. the ability to reach Extension agents on their personal account. Being able to separate work from personal life on social media accounts can be a challenge as a result of this. Sarah expressed: "People will message me on my personal page if they have 4-H questions or they have Extension questions. So, in that regard, it's very hard to separate life from work because they can message you at any time."

\section{Accessibility}

A potential barrier for utilizing social media is accessibility. It is important to make sure where, how, and what is being posting on social media platforms are accessible for all clientele. However, this can be difficult to accomplish. When asked what barrios exist with using social media, Sarah responded:

I don't personally have a barrier for using social media. But for the people that do, we want to make sure that what we're putting out there is accessible for everybody. And I don't always have the skills or personally think through like 'how do I need to make this 
post so that everybody can access it and get the information that they need from this post.' (...) How do we make sure we're reaching all audiences?

Trying to reach the population of individuals who do not have social media accounts is also a potential barrier. James mentioned: "I think we have an existing challenge of trying to reach a population of people that do not have Facebook."

A potential need for implementing social media is assuring that WVU Extension is reaching their clientele rather than their clientele reaching them. Social media can foster this accessibility when implemented properly. This creates a more positive relationship with clientele. James stated:

I think we're doing a better job of reaching our community where they are, versus making our community come to us. (...) If you can see it on Facebook and have all the details and not necessarily have to go fishing for it, I think is a lot better. (...) I think it definitely put us in a positive place compared to where we could have been without it.

\section{Responsibility}

It can be overwhelming to implement innovative social media practices while still focusing on the basis of what Extension provides for their community. Extension agents appreciate when WVU Extension communications specialists create posts for them to share. This saves a huge amount of time and assures that it is done correctly. Whose responsibility should creation and management of social media be? Sarah shared her thoughts: 
My job is not full time communications. So I'm trying to do all this other programming too. So it is a tool that I use to get information out about the programs. (...) I do always appreciate when they [WVU Extension Communication Specialists] create the content and get it out and then I can just post it to our channels. I mean, that saves a huge amount of time. Then I'm not worrying about what photo do I use and who do I tag in this, and doing it the right way.

Building on responsibility of social media creation and management, Kimberly expressed her thoughts on having a college student intern handle this. She stated:

I think I'm about to pay one of our college kids who is home for spring semester to take us to the next level. And like I said that Instagram account (...) Our next step is going to be to talk to someone who is very current with social media and figure out if they can help us get to that next level, but for now that's all we're doing is YouTube Padlet and Facebook.

Volunteers have contributed to the success of Extension programs in the past, especially 4-H club leaders. Since face-to-face communication has failed and virtual means of communication have increased, it's taken some of that responsibility away from volunteers. Sarah mentioned: "It's allowed agents to work together and take pressure off volunteers. So volunteers are encouraged to come but they don't have to plan a meeting." 
The need for volunteers has decreased as a response to the increase of virtual activities in place of in-person ones. When implementing Extension events and activities on social media platforms, such as Zoom, the need for volunteers decreases greatly. Kimberly shared:

It's hard to continue to motivate volunteers, especially whenever we are doing things electronically and it doesn't require the manpower, you know, one person can lead a zoom meeting with 50 kids. One person can't lead a service activity with 50 kids, so it reduces the need for those people.

Although the need for volunteers has decreased, the need still does exist. Volunteers that are needed might even have more responsibility as far as learning how to use the new technologies of social media platforms. Elizabeth mentioned a training that Extension agents provided for these volunteers. She stated: "All of the agents got together and provided that type of training to help leaders become more comfortable with using the technology."

\section{Too Much Online}

Work for community members and school for 4-H members became predominately online as a response to COVID-19. With Extension programs also being implemented online through social media practices, the amount of time spent online has become overwhelming for clientele. This also contributes to the difficulty of balancing the large amount of virtual work. Elizabeth said:

It was just such an overwhelming time (...) people were trying to figure out how to do this virtual learning school from home. (...) I think it was just a crazy time for folks. And they 
were trying to balance everything. (...) The kids are burnt out once they're done with their virtual schooling and they want to be outside doing something.

It has been becoming difficult to keep 4-Hers specifically engaged due to the large amount of online school they are completing. Sarah discussed: “They're trying to do school online. Like maybe they [4-Hers] just aren't interested in doing another online thing."

Taking into consideration that 4-Hers are burnt out from their online school, there is a need for adjustments when creating content. It's important to keep the activities and events fun so that the youth will want to participate after a full day of online school. Kimberly mentioned:

I think it's been tough with our kids this year going so much online school that by the time they finished all their online learning and their requirements for that they're burnt out and being on the computer. So we had to make it all really like fun in something that they wanted to participate in, and nothing that felt like regular school. 


\section{Chapter V}

\section{Conclusions}

\section{Introduction}

Outlined in this chapter are the conclusions obtained from the interviews with four West Virginia Extension agents. Implications and recommendations are included in these findings. The purpose of this study was to describe how West Virginia University Extension Service is utilizing innovative social media practices to remain connected with clientele as a response to protocol enacted by executive orders to slow the spread of COVID-19. The following objectives were developed in order to achieve the purpose of this study:

1. Describe innovative social media platforms being used.

2. Describe how innovative social media practices are being used.

3. Describe why innovative social media practices are being used.

4. Identity perceptions of barriers and needs related to utilizing innovative social media practices.

\section{Objective 1 Conclusions}

Research Objective 1 was to describe innovative social media platforms being utilized by Extension agents in West Virginia. The platforms described were 1) Facebook, 2) Instagram, 3) Padlet, 4) Zoom, 5) Twitter, and 6) YouTube.

Facebook was found to be the most used social media platform by Extension agents in West Virginia. This platform did a successful job of reaching the clientele, rather than having clientele reach Extension. This is because Facebook was already being used by the general population. In fact, Facebook has remained the most popular social media platform for over a 
decade (Pew Research Center, 2016). WVU Extension predominately used Facebook as a way to share information with the public and keep them up-to-date.

Since many in-person 4-H camps had to be canceled in 2020, Extension utilized Facebook as a way to host a virtual 4-H Camp. This was done through a private group on Facebook. All registered campers had the opportunity to join this private group where camp festivities would take place virtually. For the few campers who did not have a way to access Facebook, alternate methods were provided.

Another commonly used social media platform by Extension agents in West Virginia is Instagram. Instagram has remained the second most popular social media platform (Pew Research Center, 2016). 4-H Extension agents shared that when they use Instagram, they know they are predominately reaching youth. Since their audience on Instagram is mostly 4-Hers, Extension agents adjust their posts to fit this. Instagram stories are utilized as a way to interact with 4-Hers. Since Instagram is a social media platform used for sharing images, Extension agents often share photos of familiar faces.

Although Instagram usage did not become to exist because of face-to-face communication failing, agents have shared their increase of usage in their efforts to maintain engagement. Extension agents that are hesitant about utilizing Instagram professionally, expressed wanting assistance with making this possible. There is a plenty of room for growth and opportunity in regard to using Instagram in West Virginia Extension. With the increase of social media usage as a response to COVID-19, Extension agents are becoming more comfortable and willing to use social media for various purposes. In the future, Instagram in West Virginia Extension could significantly increase with the right tools and guidance. 
Padlet is a newer social media platform that West Virginia Extension agents have been utilizing. WVU Extension pushed for the use of Padlet at the beginning of COVID-19 as a way to virtual connect with clientele. Extension agents shared that they still have a lot to learn when it comes to utilizing Padlet, but have shared positive results so far. Padlet was predominately used in conjunction with other forms of social media as an alternative to those who did not use traditional forms of social media, such as Facebook. A major benefit to using Padlet is the level of safety is offers for participants.

Zoom is a social media platform that greatly increased because of face-to-face communication failing. Since in-person meetings and activities were no longer possible, Zoom provided an alternative. Extension agents indicated using Zoom predominately for meetings. However, virtual 4-H camp also utilized Zoom since in-person camp was not an option in some counties in West Virginia. Zoom would most likely have not been used to this capacity if it was not for COVID-19.

Twitter is a social media platform that was not often used by West Virginia Extension agents. However, agents reported that they do keep their personal twitter accounts professional. Although Twitter is not being used for updates from county Extension offices, it does have potential for being utilized in the future as social media usage increases. Twitter played a vital role in updating the public during COVID-19 (Rufai, 2020).

YouTube is another social media platform used occasionally by Extension agents. Although clientele do not regularly scroll through YouTube like they do on other platforms, such as Facebook and Instagram, YouTube allows Extension agents to share videos. These video links can then be shared to social media platforms that Extension's clientele are regularly using. 


\section{Objective 2 Conclusions}

Research objective 2 was established to describe how innovative social media practices are being used by West Virginia Extension as a response to protocol enacted by executive orders to slow the spread of COVID-19. What to post and when to post were identified as strategies used by West Virginia Extension agents in regard to implementing innovative social media practices.

Extension agents shared that they utilize photos to increase engagement with clientele on social media platforms. Relationships and connections are an important aspect of Extension. Agents found that sharing photos of community members received better responses from clientele. Doing this gives clientele a way to feel connected to others who are participating, even though it is virtual. Extension agents expressed wanting to keep the connection they once had with clientele and noted that sharing photos of familiar faces and peers, proved to be a successful way to reach this goal.

Incentives is another method Extension agents used to increase engagement and keep clientele involved in what they are doing. Incentives can be as simple as offering all participants a chance to win a gift card. Incentives provide the factor of motivation for those who may be hesitant to participate.

When scrolling through social media platforms, people often scroll past lengthy posts because time is valuable. Extension agents expressed condensing their posts and providing just enough information, but not too much. In doing this, they are meeting the needs of their clienteles' attention spans. They are also providing only the most important information so that their clientele don't have to filter through what is needed. Extension agents are always sure to include where to find any additional information. 
Extension agents found that leaving activities open ended led to clientele forgetting about them. Timeframes can be an effective way to hold clientele accountable, as well as increase responses. Extension agents include deadlines in their social media posts for the best level of engagement. Providing incentives, in addition to timeframes, can be valuable. For example, Extension agents can utilize multiple deadlines, but offer an incentive for those who meet the first deadline.

It was expressed an abundance of times that social media usage has increased greatly during the national pandemic of COVID-19. With this increase of social media usage, it's very important for Extension agents to understand when to post. Extension agents found that maintaining consistency with their posts increases engagement. If posts are consistent in when and where they are posted, then clientele know when and where to look for the necessary information.

Knowing your clientele is very important in establishing the best time of day to share posts. Social media analytics can be a valuable tool is determining this. One Extension agent found that her clientele was most engaged in the evenings. Another agent found that his clientele was most engaged in the middle of the day. Since Extension exists within all fifty-five counties in West Virginia, each county is reaching different clientele. Different clientele will have different needs. This includes what time of day they check social media and/or have time to participate in various activities and events through Extension. Understanding the best time to reach clientele on social media is very beneficial in determining when to post and gaining the most feedback as a result. 


\section{Objective 3 Conclusions}

Research objective 3 was established to describe why innovative social media practices are being used by West Virginia Extension as a response to protocol enacted by executive orders to slow the spread of COVID-19. Communication, accessibility, promotion, and engagement were identified as four main reasons why innovative social media practices were being used by West Virginia Extension.

Communication was found to be a major reason for utilizing social media when face-toface contact was no longer an option. Social media began being implemented by other businesses and industries as a method of communication with the public during COVID-19 (Geol et al., 2020). Extension agents found that a large portion of their clientele was already using various social media platforms, which made it a realistic option for remaining connected during COVID19. Additionally, other forms of communication were still being used for the small portion of the clientele that couldn't be reached on social media platforms. Integrating social media as a communication tool has led to an increase in accuracy of content, promptness, and efficiency.

Traditionally, Extension has relied heavily on in-person communication. Extension agents shared that miscommunications have occurred in the past due to passing of information verbally through various people. This has unintentionally created a game of telephone effect when disseminating information. When sharing information on social media, Extension agents have the ability to control the accuracy of the content they are sharing. They know exactly what information is being posted, such as when, where, why, how, etc. They also know when the information is being shared and who the information is reaching.

Extension agents have found this to be very useful when sharing information with 4-H clubs specifically. Traditionally, Extension agents would communicate with club leaders, then 
club leaders would communicate to $4-\mathrm{H}$ members and parents. Since in-person meetings were no longer an option during COVID-19, Extension agents resorted to posting information to club pages on Facebook. As a result of this, Extension agents found that they appreciated the control of accuracy that communicating on social media provided. This may not have been discovered if face-to-face communication had not failed as a response to COVID-19. In the future, utilizing social media to communicate with $4-\mathrm{H}$ clubs can be utilized in conjunction with in-person club meetings. Information can be shared through word of mouth during in person meetings but also be supported by what is posted on social media. This increases communication accuracy of content drastically.

Social media provides the ability to share information in a very short amount of time. In the past, Extension agents have sent post cards containing information. The turnaround time for this could easily take a week, compared to posts on social media being nearly instant. In times on COVID-19, promptness of sharing information became increasingly important for West Virginia Extension agents. Social media has played a vital role in decreasing the amount of time to disseminate information in the past year. This has shown to be extremely efficient. Since there was a lot of uncertainty in regard to Extension related events and activities during COVID-19, last minute decisions, such an cancelations, became necessary. Social media became a viable and realistic option for Extension agents to share last minute but necessary information. The response from clientele to Extension agents using social media platforms to share this information was found to be very positive.

Social media is completely online, which means that communication can exist from nearly any place at any time (Gharis et al., 2014). Extension agents shared that a major reason for utilizing social media was the lack of geographic restriction. They could reach the majority of 
their clientele regardless of location. Having no geographic restriction became predominately important during COVID-19 because face-to-face communication was no longer an option. Social media platforms provided a method of real-time communication for Extension agents and clientele.

These social media practices may not have been utilized if the opportunity for virtual communication and activities had not presented itself as a response to COVID-19. However, Extension agents found that the accessibility social media provided was beneficial. This accessibility proved to be very timely. Time is extremely valuable for everyone. Decreasing the amount of time used to disseminate information resulted in positive feedback from both Extension agents and clientele.

Extension emphasizes creating positive changes by sharing knowledge gained through research and education with community members (USDA-NIFA, 2020). Promoting this knowledge is a necessary aspect for Extension agents. As a response to face-to-face communication failing, these methods of promotion on social media platforms have increased greatly by WVU Extension agents. As a response to this, Extension agents discovered that they have reached new audiences. Incorporating the use of social media in Extension can potentially reach individuals who are not previously familiar with the Extension Service (Cornelisse et al., 2011).

Additionally, integrating social media has unintentionally become a recruitment tool for the 4-H side of Extension. West Virginia Extension agents shared that their increase of promotion of 4-H on social media platforms has led to an increase of new members. Agents shared that they have been posting virtual activities and events on social media platforms more during COVID-19. Since these posts are able to be shared by 4-H members and families, they are 
reaching new people. These individuals may not have discovered 4-H if it wasn't for seeing it on social media platforms. Additionally, social media usage may not have increased as quickly or at all if it wasn't for face-to-face communication failing as a response to COVID-19. Promotion of 4-H on social media has proven to be a valuable tool in recruitment.

Engagement is an aspect of Extension that agents value. Traditionally, engagement in Extension happened in person through programs, activities, and events. With this no longer being possible during COVID-19, West Virginia Extension agents began utilizing social media practices to remain connected with their clientele. The primary goal for doing this was to keep the level of engagement that traditionally took place in person. Extension agents found that social media contributed greatly to maintaining this level of connection and engagement.

\section{Objective 4 Conclusions}

Research objective 4 was established to identify perceptions of barriers and needs related to social media platforms being utilized by Extension agents in West Virginia. These perceptions of barriers and needs were found to be 1) Protection Issues, 2) Separating Work from Personal Life, 3) Accessibility, 4) Responsibility, and 5) Too Much Online.

Child protection issues is not a new issue, but it is still very relevant. It may be even more relevant now that social media is involved. Extension agents expressed that they found sharing photos of familiar faces, such as 4-Hers and community members, got higher responses. It's important that Extension agents have permission to share photos of every person included in pictures. Knowing and following these guidelines is extremely important, especially on social media platforms. 
Since certain 4-H members' photos can't be shared, this could create a division amongst members. Younger kids may not understand why their friends' pictures are being shared and not theirs. Trying to remain inclusive of everyone in regard to sharing photos on social media is a potential barrier.

ADA compliant guidelines should also be followed when utilizing social media platforms. Extension agents have to include all words in the description portion when posting a photo. This is not something that is new, but is something that will need to be utilized more with the increase of social media usage in Extension. Additionally, WVU Extension will need to be aware of the development and changes of ADA regulations in the future.

A major barrier of using social media for Extension purposes is safety. Although this has not been a known issue for WVU Extension agents, it should be taken into consideration when utilizing social media. Knowing what information to post is very important moving forward with the increase of social media usage. This is especially important with the youth (4-Hers) that are involved. Measures should be taken to ensure the safety of everyone.

Being able to separate work from personal life was found to be a potential barrier for implementing social media within Extension. Since face-to-face communication was no longer an option for Extension as a response to COVID-19 protocol, Extension heavily increased their social media usage. Clientele also increased using social media platforms as a way to communicate with Extension agents, regardless of time or day of the week. With Extension agents receiving questions on their personal accounts at all hours of the day, it becomes difficult to distinguish between work and personal time. Efforts should be made to find ways to aid Extension in separating work from personal life when using social media for work purposes. 
Although a large amount of the population is using social media in their personal lives, there are still individuals out there who do not use social media platforms. Even though Extension agents discussed this as a relatively small population of their clientele, reaching this audience is a potential barrier. Extension agents do use other means of communication, such as emails and postcards. However, the pandemic of COVID-19 lead to the need for real-time communication with a fast turn-around. Social media platforms provided this for Extension during this time of need.

Extension agents have not traditionally been taught or required to implement social media as a way to communicate with their clientele. The amount of time and skill required to implement social media into their programs is a potential barrier. Extension agents expressed their appreciation for when WVU Extension Communications specialists create the posts for them to share. With this increase of social media usage in Extension due to face-to-face communication failing, where does the responsibility lie for managing this? The need for experts to help implement social media for Extension agents could be a potential need moving forward with utilizing innovative social media practices in WVU Extension.

Volunteer responsibility is also an important aspect to discuss. The decrease in face-toface communication has also led to a decrease in the need for volunteers. For example, less people are needed to manage online activities and events than in-person. However, when volunteers are needed for online events, their responsibility increases. If a volunteer is hosting a Zoom meeting, they need to be well versed on utilizing that social media platform in order for it to be successful. Some agents have even hosted trainings for volunteers on how to use various social media platforms. 
When face-to-face communication failed as a response to COVID-19 protocol, schools, businesses, and other organizations began working remotely. The increase of online school has become exhausting for students (Collie, 2021). Extension agents found that 4-H members seem burnt out on being online by the time they get finished with their online schoolwork. This is a potential barrier to utilizing social media in Extension. In order to keep 4-H members engaged in the online activities and events, Extension personnel need to resort to creation of innovative content and methods when utilizing social media.

\section{Discussion and Implications}

The purpose of this study was to describe how WVU Extension Service is utilizing innovative social media practices to remain connected with clientele when face-to-face communication has failed. The opportunity to research this failed communication is the response to protocols enacted by executive orders to slow the spread of COVID-19. Extension agents expressed their increased use of social media during this time. Agents also shared that they feel their clientele have gotten used to looking on social media platforms for various information. As protocols established to slow the spread of COVID-19 begin decreasing, West Virginia Extension agents will be able to have in-person events and activities again in the future. The circumstances that lead to having to rely on social media as a major form of communication in Extension was unfortunate, but also produced valuable results. Moving forward, WVU Extension will be challenged to utilize these innovative social media practices in conjunction with face-toface communication.

The framework for this research was Rogers' (2003) Diffusion of Innovations. It's important to note that the social media platforms are not considered to be innovative for this 
study; however, the social media practices being implemented by West Virginia Extension agents are innovative, however. This study described how West Virginia Extension agents were utilizing these innovative social media practices when face-to-face communication was no longer possible. The innovative social media practices being implemented by the population for this study will likely see an increase in adoption by other West Virginia Extension agents, based on the five attributes of an innovation outlined by Rogers (2003), which is relative advantage, compatibility, complexity, trialability, and observability.

Extension agents shared strategies pertaining to how and when they post content to social media. They also discussed how they have used various platforms for different purposes. These methods have proven to be effective in reaching their clientele. However, the needs of clientele may differ from county to county. There are tools, such as social media analytics, that share what posts get the most feedback as far as likes, comments, shares, views, etc. This is a valuable tool in determining what time to post to which platform, as well as what content to post. As an Extension agent, knowing your clientele is extremely valuable. Reaching clientele at a time and a platform that is convenient for them is necessary in increasing engagement and producing results regarding social media usage in West Virginia Extension.

An aspect of 4-H that the youth look forward to every summer is 4-H camp. Many counties in West Virginia had to host virtual 4-H camps in 2020. Extension agents shared how social media platforms, predominately Facebook and Padlet, made virtual camp possible. With the spread of COVID-19 slowing, in-person 4-H camps will hopefully be possible again in the near future. This new method of virtual 4-H camp could possibly be incorporated with in-person camp moving forward. This would allow campers who have other obligations during the summer and cannot attend in-person camp, the opportunity to still participate. 
Extension agents using innovative social media practices were selected for the purpose of this study. These agents discussed their increased social media usage as trial and error methods. Some agents even expressed the want or need for learning more about best practices for implementing social media for work purposes. Considering that the agents who are using innovative practices expressed this need, it can be inferred that most West Virginia Extension agents have a greater need for this.

WVU Extension Service has provided workshops and trainings related to implementing social media. As innovative social media practices increase in the future, so should assistance in developing knowledge and skill to implement these practices. There is a need for communication experts to aid in creation and management of social media accounts and practices. There is potential need for new positions within WVU Extension specifically for managing county social media accounts.

Overall, the national pandemic of COVID-19, led to an increase of social media usage within West Virginia Extension. As a result of this, WVU Extension agents found innovative and effective social media practices to maintain connection with their clientele. Knowing and understanding these effective social media practices will be valuable for the future of social media usage within WVU Extension.

\section{Recommendations}

The first recommendation for future research is to interview more Extension agents in West Virginia. This study selected participants whose survey results indicated high use of innovativeness on social media platforms. The survey had a 50\% response rate. Although this is very high, half of the population was not initially considered. To account for this gap, participant 
selection was checked by an expert in the field and additional recommendations were considered. There could still potentially be innovative social media practices used by WVU Extension that were not described in this study.

Another recommendation for future research is to analyze quantitative data as well. For the purpose of time for this study, the researcher developed a survey instrument strictly for gathering information needed to select participants. The survey instrument implemented in this study could be developed and manipulated in future studies for the purpose of collecting quantitative data for analysis. In doing this, the researcher would need to conduct additional pilot studies to increase reliability and validity of the instrument. Results from the survey could be analyzed and reported in conjunction with interview findings making the research a mixed methods study.

Replicating this study in other states is another recommendation for future research. The Extension Service exists in all 50 states (USDA, 2020). This study is beneficial in the future development of the Extension service in West Virginia and could be beneficial in other states as well. Research between various states in the U.S. could be useful for the Extension Service as a whole.

Another recommendation for future research is analyzing the needs of clientele for the Extension service in the state of West Virginia. Since this study found that knowing the best time and platform to reach clientele is necessary in maintaining engagement, this could be beneficial in implementing social media in the future. It's also important to note that different counties across the state will have different needs. Understanding clientele needs of WVU Extension pertaining to social media is valuable moving forward with social media usage increasing in the future. 
In completing this research and providing recommendations for future research relating to social media usage in WVU Extension, the researcher hopes that Extension agents in West Virginia will feel comfortable and confident with implementing innovative social media practices in the future. 


\section{REFERENCES}

Auer, M. (2011). The policy sciences of social media. Policy Studies Journal, 39, 709-736.

Bardon, R. E., Hazel, D., \& Miller, K. (2007). Preferred information delivery methods of North Carolina forest landowners. Journal of Extension, 45(5), 1-12.

Brügger, N. (2015). A brief history of Facebook as a media text: The development of an empty structure. First Monday.

Centers for Disease Control and Prevention (CDC). (2020). Coronavirus Disease 2019. Centers for Disease Control and Prevention. Retrieved from https://www.cdc.gov/coronavirus/2019-nCoV/index.html

Collie, R. J. (2021). COVID-19 and Teachers' Somatic Burden, Stress, and Emotional Exhaustion: Examining the Role of Principal Leadership and Workplace Buoyancy. AERA Open, 7, 2332858420986187.

Cornelisse, S., Hyde, J., Raines, C., Kelley, K., Ollendyke, D., \& Remcheck, J. (2011). Entrepreneurial extension conducted via social media. Journal of Extension, 49(6), 5.

Diem, K. G., Hino, J., Martin, D., \& Meisenbach, T. (2011). Is extension ready to adopt technology for delivering programs and reaching new audiences. Journal of Extension, $49(6), 1-7$.

Dillman, D. A., Smyth, J. D., \& Christian, L. M. (2014). Internet, phone, mail, and mixed-mode surveys: the tailored design method. John Wiley \& Sons.

Edward, Luke. (2020). What is Padlet and How Does it Work for Teachers and Students? Tech Learning.

Fogel, S. (2010). Issues in measurement of word of mouth in social media marketing. International Journal of Integrated Marketing Communications. 
Gharis, L. W., Bardon, R. E., Evans, J. L., Hubbard, W. G., \& Taylor, E. (2014). Expanding the reach of extension through social media. Journal of Extension, 52(3).

Goel, A., \& Gupta, L. (2020). Social Media in the Times of COVID-19. Journal of Clinical Rheumatology.

Kaplan, A.M. and Haenlein, M. (2010). Users of the world, unite! The challenges and opportunities of social media. Business Horizons

Landry, R. B. (2010). Developing and building collaborations between a cooperative extension service and a local school and its community. Journal of Extension.

Lincoln \& Guba, E.G. (1985). Naturalistic Inquiry. Newbury Park, CA: Sage Publications, Inc.

Mayo Clinic Health System. (2020). COVID-19: What is it, what you need to know. Mayo Clinic Health System. Retrieved from https://www.mayoclinichealthsystem.org/hometownhealth/featured-topic/coronavirus-what-it-is-what-you-need-to-know

Nadkarni, A., \& Hofmann, S. G. (2012). Why do people use Facebook?. Personality and individual differences, 52(3), 243-249.

National Conference of State Legislatures (NCSL). (2020). President Trump Declares State of Emergency for COVID-19. NCSL Retrieved from https://www.ncsl.org/ncsl-indc/publications-and-resources/president-trump-declares-state-of-emergency-for-covid19.aspx

Padlet. (2021). Padlet Home. https://ja.padlet.com/about

Pew Research Center. (2016). Facebook Remains the most popular social media platform. Pew 
Research Center, Internet \& Technology

https://www.pewresearch.org/internet/2016/11/11/social-media-update-2016/pi_2016-11-

11_social-media-update_0-01/

Rauniar, R., Rawski, G., Yang, J., \& Johnson, B. (2014). Technology acceptance model (tam) and social media usage: an empirical study on facebook. Journal of Enterprise Information Management, 27(1), 6-30.

Rufai, S. R., \& Bunce, C. (2020). World leaders' usage of Twitter in response to the COVID-19 pandemic: a content analysis. Journal of Public Health, 42(3), 510-516.

Sauermann, H., \& Roach, M. (2013). Increasing web survey response rates in innovation research: An experimental study of static and dynamic contact design features. Research Policy, 42(1), 273-286.

Strauss, Valerie. (2020). As school rapidly moves online across the country, concerns rise about student data privacy. The Washington Post.

Twitter, Inc. (2021). Twitter About. https://about.twitter.com/

United States Department of Agriculture (USDA). (2020). Cooperative Extension History. USDA National Institute of Food and Agriculture. Retrieved from https://nifa.usda.gov/cooperative-extension-history

WVU Extension Service. (2021). About Extension. https://extension.wvu.edu/about YouTube. (2021). YouTube About. https://www.youtube.com/about/ 


\section{APPENDIX A}

\section{Survey}

\section{Start of Block: Welcome}

Q3 Welcome to this survey, which will examine WVU Extension Service's efforts to remain connected with clientele through social media platforms while adhering to quarantine protocol enacted by COVID-19. This survey will take a maximum of 10 minutes to complete. Responses are highly valued because the results from this survey will be used to select individuals to participate in interviews. For this purpose, please complete the personal demographics section accurately to ease potential future contact. This survey is being administered to all Extension agents and agents in training in West Virginia. Privacy will be protected and confidentiality will be maintained. Only a qualified research team will have access to the responses. If you have any questions, please email Madison Quinn at mbq0001@mix.wvu.edu. To begin the survey, click the arrow below. 
Q19 Do you utilize social media as a communication tool to stay connected with clientele?

\section{Yes (1)}

No (2)

Q11 Has quarantine protocol during COVID-19 affected how you remain connected with clientele?

Yes (1)

Maybe (2)

No (3)

Skip To: End of Block If Has quarantine protocol during COVID-19 affected how you remain connected with clientele $?=$ No 
Q12 Has your social media usage increased due to regulations in place for COVID-19.

Yes (1)

Maybe (2)

No (3)

Skip To: End of Block If Has your social media usage increased due to regulations in place for COVID-19. = No 
Q14 Select how often you used the following social media platforms for work purposes BEFORE COVID-19 occurred

\begin{tabular}{l|ccccc} 
& Once a week & 2-3 times a & 4-6 times a & Daily (5)
\end{tabular}

\section{Facebook (1)}

Instagram (2)

Padlet (3)

Twitter (4)

YouTube (5)

Snapchat (6)

If other, please

specify (7) 
Q16 Select how often you use the following social media platforms for work purposes while adhering to quarantine protocol DURING COVID-19

\begin{tabular}{l|cccc} 
& Once a week & $2-3$ times a & 4-6 times a & Daily (5) \\
Never (1) & $(2)$ & week (3) & week (4) &
\end{tabular}

\section{Facebook (1)}

Instagram (2)

Padlet (3)

witter (4)

YouTube (5)

Snapchat (6)

If other, please

specify (7) 
Q24 Please rate the following statements pertaining to social media use DURING COVID-19 


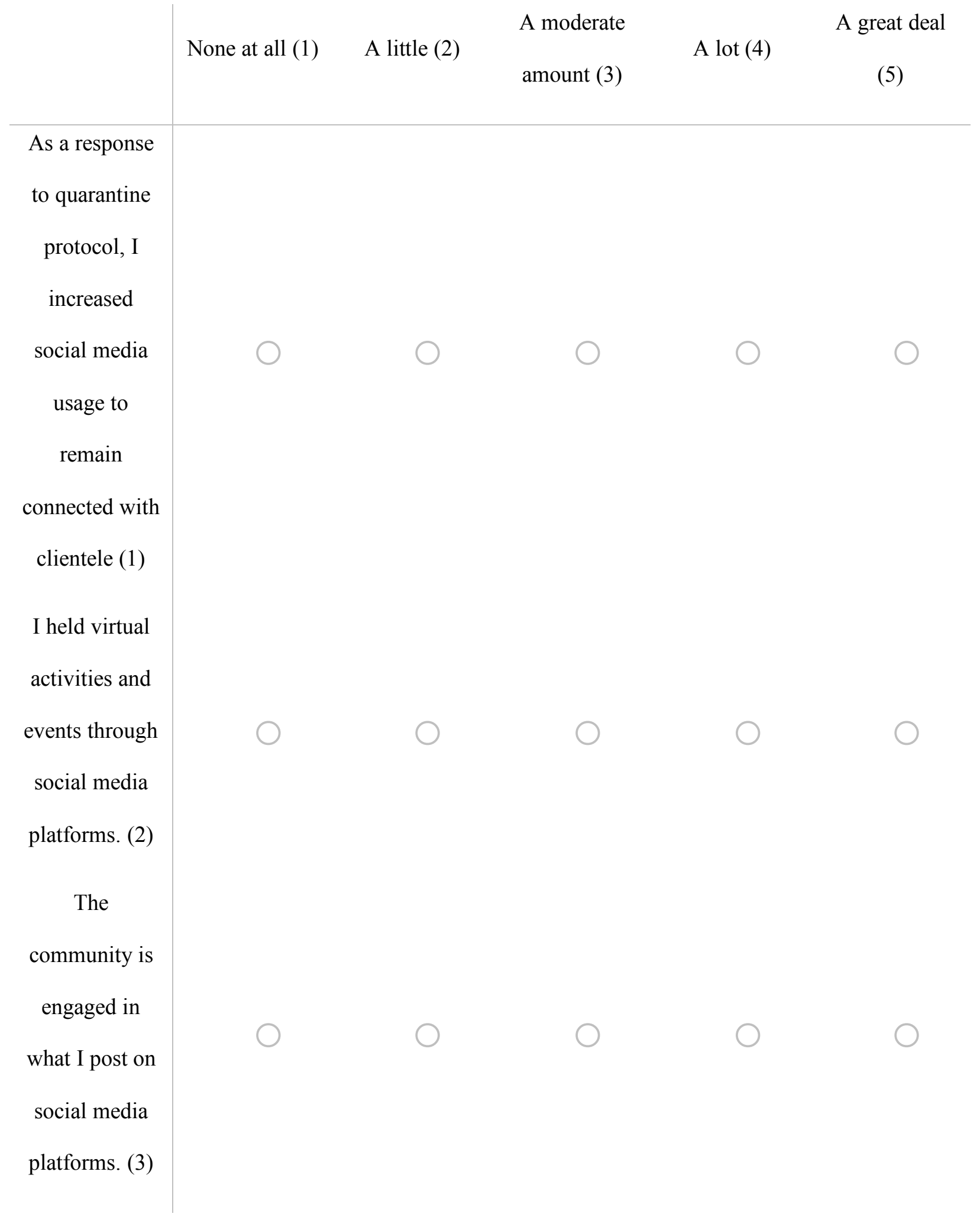




\section{I communicate \\ with \\ community \\ members using \\ social media \\ platforms (4)}

Q7 What county are you Extension personnel?

County (1)

V Barbour (1) ... Wyoming (55) 
Q9 Check any positions that currently apply to your job description

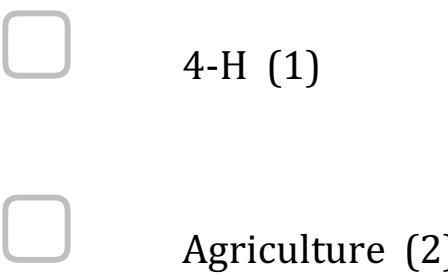

Family and Community Development (3)

Q21 Please fill out the following information as accurately as possible to ease potential contact in the future for interviews.

Q20 Name:

Q22 Phone Number: 
Q23 Email Address:

Q5 Thank you for your participation in this survey that examined WVU Extension Service's efforts to stay connected through social media platforms while adhering to quarantine protocol enacted by COVID-19. The results from this survey will be used to select agents to participate in interviews in the near future to collect qualitative data. If you would like to change any responses, use the back arrow on the left. If you are ready to submit, use the arrow on the right below. 


\section{APPENDIX B}

\section{Interview Guiding Questions}

1. Can you describe what you would consider to be beneficial social media practices that you implement for work purposes?

2. When was your clientele most engaged with your social media posts and how could you tell?

3. What are some key examples of times you utilized social media to remain connected with clientele as a response to COVID-19 protocol?

4. What was something you thought would go well but didn't, and why?

5. Your survey response indicates that you used these (Facebook, Padlet, Instagram, Twitter, Snapchat, YouTube) social media platforms to remain connected with clientele.

a. Provide an example of an innovative way you used each platform.

b. Describe how you used different platforms for different purposes.

6. Social media allows Extension to reach new audiences, potentially individuals who are unfamiliar with Extension.

a. Can you share an experience that supports or denies this statement?

b. Has this become predominantly more true during COVID-19? Why?

7. What do you hope your clientele gains through your efforts of utilizing social media, and why?

8. How is social media usage for work purposes changing and how can you adapt to this? 
9. Describe any challenges you foresee needing to overcome in order to continue utilizing social media practices in the future?

10. Describe how you work within guidelines for using social media platforms.

11. Do you consider yourself an innovator when in regards to social media practices and why?

12. Can you describe a time you noticed a WVU Extension agent (other than yourself) utilizing innovative social media practices that you found beneficial.

13. Do you have any additional information pertaining to my research you'd like to add or any questions for me? 


\section{APPENDIX C}

\section{Transcribed Interviews}

\section{Sarah}

Madi: Can you describe what you consider to be beneficial social media practices that you implement for work purposes?

Sarah: Sure, um so I am a county agent based in Wetzel County and um I cover all the units of extension but I'm primarily focused on 4-H youth development

so social media have been, ya know, a big part of communication both with 4-H parents and 4-H youth Um. Our two social media tools are Facebook and Instagram I'm going to apologize because I hear my dogs coming up so, ya know, hopefully they don't make too much noise. Um (giggles) Yeah so Facebook and Instagram. And what's been beneficial is that we can push out information at all hours of the day And we know, I mean, I am friends personally with a lot of the 4-H parents so I know they are on Facebook. I know they are using it. So, um, it's been an easy way for me to know they are getting the communication that I am sending them because they can't say "oh no I never saw it" cause I know you're on Facebook. Um, and our clubs use it to communicate as well. So most of our clubs have their own group or page on Facebook so they can plan events um or just talk amongst their members, um. And then I, as the agent, can communicate county level information or state level information to those clubs.

Madi: Okay. And have you had any issues with clubs getting on board with that? Or do they seem pretty much like they picked it up and just went with it?

Sarah: Yeah, actually yeah. All those clubs, pages, um, existed when I started as an agent. So I've been an agent for six years. And, all those clubs were.. Some were more active than others. But they all had a Facebook presence when I started. Um. Some clubs use it more than others, especially, there's one in particular, they just do not have good broadband, good internet access where they are in that area of the county. So that club is not as active in seeing information on Facebook as some of the others. But they do use it. And I haven't gotten.. I haven't gotten pushback. There are several families that I know of that don't have Facebook. They don't use it. And so I always try to be conscious of that shouldn't be the only way we are communicating. I wanna make sure that we are using other methods too, ya know, we will send emails. Um. Everybody who's in 4H has to register through an online system so they have to have an email address to join $4 \mathrm{H}$. Um Ya know. Generally a parent email address. So we push out information that way. And also like we'll just do. We'll send out postcards. Or we'll send out letters in the mail. Uh so even if they aren't on social media, we can still get them the information. But, in general when something quick comes up, that's such an easy way to send it, send it out.

Madi: Yeah, and that's good that a lot of your clubs are on board with that too. I didn't think about that. The clubs having a facebook page and being able to communicate with the whole club instead of just like person to person on those levels. 
Sarah: Yeah. And it's been uh, ya know, kinda as in the agent role, I think about how things get communicated and I want them shared in a certain way. And so ya know traditionally we would have like a meeting with all the club leaders. I would share information with them and then they would distribute it out to their clubs. Well then you don't have control over how that message is shared and what information is getting shared with parents and kids. If i'm the one posting it to the club page, then I know exactly what I'm saying and making sure all the details are there and not really having that middle person uh between the information and getting to the families. So that that's been useful

Madi: So do you think that utilizing social media in that instance has kind of like bridged that gap where like miscommunications might have occurred in the past?

Sarah: I think so. Um. And not even that there have been miscommunications all the time but maybe the the promptness of when things are shared because traditionally clubs would only meet once a month. And that's if everything went perfectly well.Ya know, there wasn't any weather that might cancel a meeting. Or, ya know, basketball games seem to always give people reason not to go to meetings. So ya know, they didn't all have to come to the firehall on a Sunday afternoon to get this information. We can share it with them at any time. And, I mean, certainly that's been helpful this year when they haven't.. they have not been having in person club meetings. So we can, ya know, we can share it with them that way.

Madi: Have they been doing virtual club meetings or do you know is it depending club to club, or do you know how they are doing that this year?

Sarah: Yeah, I mean, I can only speak for Wetzel County. Across the state seems to be a little different. Um. In Wetzel county they have not been, really no in person events. There. I don't even know (connection error) clubs had virtual meetings. Regional basis we are calling it the NoPanchallange for the northern panhandle. Um. And we get together once or twice a month. And any 4 Her from Wetzel Country all the way up through Hancock county is welcome to come to those meetings. Um. And that's kind of taking the place of club meetings because we aren't able to meet in person. So, ya know, a geographical restriction doesn't matter anymore. We can meet, ya know, anybody can get on zoom no matter where you are. Um It's allowed agents to work together and taken some pressure off volunteers. So volunteers are encouraged to come but they don't have to plan a meeting. Um. So and we've had a.. we've had a fairly good response to it. It's certainly not, um we're not at normal numbers. We've seen a big dropoff. But that's very common. I mean, it's not just this area, it's across the state. And really across the nation we are seeing that too.

Madi: Okay. Another question.. When was your clients held most engaged with your social media posts and how could you tell?

Sarah: Hmm.. I would say the posts that get the most response have, have photos, um first of all, and then photos that are of people they know. So it either volunteers or kids, especially if they are getting any kind of recognition for something, ya know, something really interesting that they've done. Um. An example would be when we had.. I don't know if you're familiar with the Allstar program, which is through $4 \mathrm{H}$. 
Madi: Yes.

Sarah: Okay, Um. When this year we had four new people inducted into Wetzel County as Wetzel County Allstars. And that post got a lot of um a lot of likes, shares, comments. And that was, that was on Facebook. I think our Instagram tends to get about the same likes per post. It doesn't seem to drastically change. Um. The ones that I know that get a lot more. Well i've noticed that they are um like collage posts seem to get a lot more. And I don't know if its because they see more kids faces in those, especially after camp um or a large event where I share like multiple photos at once. Those seem to get a lot of feedback. And I've been using stories on Instagram more and more and anytime I ask a question, like one of those poll questions, I see 4Hers respond to those.

Madi: Okay, awesome! Now have you noticed that different people are liking posts compared to Instagram and Facebook. Like, younger generation on Instagram, older generation on Facebook. Have you been able to notice that in your posts?

Sarah: Yeah, absolutely. Uh. That and, actually a couple years ago I gave a presentation on this for $4 \mathrm{H}$ agents. Um. Facebook is how I communicate when I want adults and parents to know something that's going on. Um. So I communicate.. Ya know, I keep that in mind when I do a post for facebook who the audience is, who's seen it. Generally it's 4H families that I'm pushing that information to. Um. Posts on Instagram, I definitely notice that more, not that there aren't parents or adults seeing it, but all my 4Hers see it. Um I know, I know that the kids themselves are seeing it. So, you know, sometimes the language changes. Sometimes, the um, the tone changes. Sometimes, like I said, I use stories more because that seems to get a little bit more interaction and not just like a like on it. Um. Yeah, so definitely two different audiences. I have noticed, we've had Instagram probably for four years I would say. And I've noticed an increase in volunteers and parents on that page just overtime. It used to be that only the kids were there. And now that, that audience is changing a little bit. But. Yeah, so, you're right on with that question.

Madi: And what are some key examples of times that you utilized social media to remain connected with clientele as a response to quarantine protocol?

Sarah: Um. Can you uh, can you repeat that because it kind of broke up a little bit.

Madi: Yeah. So what are some key examples of times that you utilized social media to remain connected with clientele as a response to quarantine protocol?

Sarah: Um. I mean, certainly we've continued to share information. Really like a daily basis we are posting things. I would say that probably, I could think of several things. The first was our, our camp this year went all virtual and so we did a lot of promotion on social media prior to that happening. And then as camp was going on, we had a page that was specially for camp. And that was combined with another county. So everybody who was participating in camp could post to that Facebook page and show what they were doing. Because there were activities that we asked them to do at home. So it was like "go for a hike and show us a picture of where you went" or 
something like that. And they could share that on Facebook. Or they did some crafts and they could share that on Facebook. So we really utilized social media as a tool for during the week of camp. And then posting, sharing some of those photos out to kind of the broader pages just to kind of promote "this is what we are doing" "We didn't have camp this year, we did this instead. Look at all of our 4Hers participating." Um. And so that got certainly not the kind of response that a normal camp does. Ya know, we only had about 35 kids participate rather than 200. But the kids who did participate, we saw really high engagement. Ya know, daily, multiple posts a day from them. Very engaged with the activities. Um, another time we used social media was when the first, I think it was in July, it was maybe the first statewide mask order came down from the governor. Um. And I just kind of put out like a "show us your mask challenge." I don't know exactly how I worded it. Um, but like I said "send us a selfie with you in a mask" and everybody who did that like I reshared all those photos a couple times a day and then if they did that we sent them like a $4 \mathrm{H}$ sticker or something just for participating. And it definitely like built upon itself, from what I saw, just like we started with one photo of just me and our STEM ambassador. Um, I don't know if you know Devaan Fox, she's in undergrad also. (Madi: "yes") So it was the two of us with our masks on and I got a couple DM's throughout the day with kids with their masks on and I shared those in the evening and that just like..then I got a flood of more. Like "Oh I see my friends wearing their masks and so like, I'll send you a photo of me wearing a mask." And so it just like built on itself for like a week. We were posting lots of photos of kids and volunteers and former ECI's or stem ambassadors or just like friends of Wetzel County $4 \mathrm{H}$ with their masks. So um, that, it certainly wasn't something I put a lot of thought into but it really.. We saw such a great response to and so I was kind of proud of that social media post (laughs).

Madi: yeah, that one is fun. Um, let's see.. Next question.. What was something you thought would go well but didn't, and why? So kind of the opposite of what you just answered (laughs)

Sarah: Yeah (haha). Um, well I'll use yesterday, like yesterday could be an example. We have been doing um, once a month or twice a month, we've been having zoom meetings for, like I said this whole northern panhandle, um, and we've had a fairly good response rate. I would say about 20 4Hers sign into those each time we've done one. Yesterday, Devaan Fox was going to teach them how to make ice cream. Like ice cream in a bag, you've probably seen that activity before. So she was doing that. She was going to do that with them. And I thought "oh this is great! Wetzel County kids know Devaan. A lot of Marshall County kids know Devaan. They'll wanna see her. And we're making ice cream. Who doesn't love ice cream!" And so, ya know, we promoted it on Facebook, you know - multiple posts. All about ice cream and Devaan. And other counties did too and put all this stuff on Instagram. And we had 7 kids. And so it was like "awh." It was like a low, ya know, it was like such a drop off from what we'd seen on these previous events. And I actually just had a meeting before talking with you, with the other agents, and we were kind of brainstorming like "oh, why didn't it go well" or "why didn't we see the turnout we've normally had?" And I don't really know, ya know. We did a lot of the same social media promoting. And yeah, didn't go great (laughs)

Madi: When you do activities like that, do the parents typically supply the supplies for that or is that something that they can say like "hey, I wanna do this" and you guys will send them the supplies. How does that usually work? 
Sarah: We've only done a couple like that, where there are like ingredients that they need. Um, but they've been in charge of supplying it themselves. So we will send out, um. Like all the social media posts had, it was either like a graphic that had the ingredients listed or we put it in the comment or the caption what they needed. We also sent emails out to all the families that were registered in $4 \mathrm{H}$. And that had the zoom link and here's the ingredients you need. So, and we've told them they can either cook along with Devaan. Like make it along with us, like a cooking show. Or, ya know, we'll send you the list and the recipe and you can do it on your own time. You can watch along with us, but you don't have to do it right then. Um, and there's always a mix. There are some kids that like, we sign on, and they're in their kitchen and they're ready to go! Um, and other times you can just tell they're watching and, you know, that's great too. But no, we have not sent them ingredients.

Madi: Okay. Do you um.. Now you do those on zoom, correct?

Sarah: Mhm

Madi: Do you usually post them to the pages afterwards, like the whole video. Or is it kinda just like log into zoom and that's the opportunity for them to be a part of it?

Sarah: That really is. Um. That's certainly an option to think about is, is doing a recording and then we could email it out. There is some.. There's some child protection issues that we have to be careful of. Like we don't share the zoom link publicly. Like I don't put the link on Facebook. We say, ya know, whenever we promote it, we say 'here's when it's happening. It's on zoom. If you are a registered 4Her, you will get an email with the link. And so we can kind of control who it.. Who the link is going to, just because we don't want just anybody signing in.

Madi: Yeah

Sarah: Yeah, so we kinda have to be careful of that. Now the recording, I mean, that's a good thing to think about - that we could email it afterwards. Um.

Madi: And you would have to um probably not include the kids in it and just do it as like uh whoever's doing the demo or something like that. I know I've seen other places doing like Facebook lives. Um. I don't know if you've used that at all, but that's another cool thing too. So.

Sarah: Yeah. We've used Facebook live. Let's see, uh. We did it once or twice at the farmers market this summer. We were doing like a little STEM demo, probably before they shut down what we could do, probably. Um. And we did a $4 \mathrm{H}$ enrollment, a drivethru at the office. And I did a little live before that. And that did, now that you say it, that did seem to get a lot of views. So that is something to think about in the future.

Madi: Yeah. The other thing I notice about what Facebook live does is, at least for me, it sends me notifications (laughs)

Sarah: Yeah 
Madi: And it'll be like "Hey, so and so is live." Um, so it's kind of a cool little reminder tool that I know I've seen um, not just Extension agents using, but a lot of different businesses and people throughout um COVID specifically as a way to stay connected. So.

Sarah: Yeah, absolutely. I support, like as agents, beyond 4H, I support the farmers market in town. Um. And we had a new market manager this year and she, like the first day, we talked about like "let's go live to show people" because it was a drive, we had to do it as a drivethru, and we wanted to like show them where to drive and kind of explain it since it was new for this year. And she did that, and it, it got such good response that really I did encourage her to like "oh, you should do it again this week!" And then it just really became her thing. Every week she would go live right at the beginning of the market. Um. And it's stopped being about how to drive through it and it was more like "okay, this vendor has tomatoes this week" and "this vendor has cornbread" and "this vendor has this." And like really showing that off. And yeah, I think people looked forward to that every week. To see what was there and then, um, we even had comments from people saying "oh, I saw you on Facebook so I came down to the market."

Madi: That's awesome, yeah. Okay, let's see.. I've got my questions pulled up here. Okay, so looking at your survey results, and I know we've talked about this a little bit so I don't want to repeat too much. But you identified that you use Padlet, Facebook, and Instagram. We talked a little bit about Facebook and Instagram, but could you just maybe give me a little example fo the way that you use Padlet.

Sarah: Sure. That is what we're using in conjunction with those regional zoom meetings. And, again, we're calling it the NoPanchallange. Each month we've put together, and I say we - so it's myself and the four other agents in the Northern Panhandle region. Um. We write eight challenges, so they are 8 little tasks that kids can do um at home, or on their own. And those get posted to the main Padlet page. So, and it. I don't know if you are familiar with it, but it does, it has a similar layout to I think Facebook. That's what I tell parents at least. Um. So they can see what the challenge is and then their challenge is to do two of those a month and then take photos and share it on the bulletin board page, which looks very similar to Facebook. You know, they can make a - post a photo and write a little comment and then other people can comment on their photos. Um. And we have seen a very good response there. So kids can do that anytime that works for them, they don't have to go to a club meeting to do it. Um, they can do as many as they want. We challenge them to do two a month, but there are 8 options there so some kids have done more than that. Um, so I don't know if you want examples of what those are. But they are.. Um.. that's kind of the tool we are using in place of in person meetings this year.

Madi: Okay. And when you post.. So let's say that you are posting things on Padlet, do you also send out emails or post them on Facebook or do you kind of keep those different things separate when you are doing your social media posts?

Sarah: Um, we have used uh.. Because the Padlet, we are trying to keep that.. We're not really sharing that link publicly. So they have to be a registered 4 Her to join that Padlet. Um, again, for child protection issues because kids are posting photos of themselves on Padlet. Um so we 
haven't shared the link publically, but we do talk about the, you know, NoPanChallange is going on. The December challenges are uploaded. Be sure to check Padlet or check your email.

Madi: Okay

Sarah: Yeah, we've taken, like I've used a couple screenshots from Padlet as photos for Instagram or Facebook, just to kind of help promote it.

Madi: Okay, cool. Umm.. we kind of answered that one. Okay, also, another point. When I was doing research, I read that social media really allows businesses, or whatever it may be, specifically Extension in this case, to reach new audiences. Um, which could potentially be individuals who maybe aren't familiar with Extension or aren't in $4 \mathrm{H}$. And, um, can you maybe share an example of why you would agree or disagree with this claim? Something that either supports it or denys it based on your personal experiences.

Sarah: Um, I would, I mean I guess I would say I support it, especially if you've seen some research about it already (laughs). Um, no, we have seen this year, I would say, we have seen a drop off in our traditional 4Hers who have been members for a long time. But we've seen an increase in new members. And specifically younger kids. So, um. I would say that like 6 to 9 age group, we've seen a big increase there. And I don't have my enrollment numbers in front of me, but um, I just know those much younger kids, we have a lot more sign ups. And I think it's because we've seen.. I've seen some new parents see posts on Facebook and we talk about "we're doing things online" or "you don't.. You don't even have to be in Wetzel County to join our program because it is online." So we border Ohio and I have two new kids who joined who live in Ohio. And normally they probably wouldn't have done that, but it doesn't matter where they live for what we're doing right now because they can do things at home and they can join us um on zoom they can join us online. So it has it has reached some of those new kids.

Madi: And do you think that it's done that specifically because of um what you've been doing to stay connected as a response to the quarantine protocol and stuff like that. Do you think that it kind of has been um a little bit of a direct effect or do you think that maybe this started before quarantine or hard to tell?

Sarah: I.. It may be a little hard to tell. I think this year especially we've really pushed $4 \mathrm{H}$ enrollment more so um than normal. Because I would say traditionally it has been the club leaders come to the Extension office. They get the information. They go to their club meetings. They have all the kids sign up at the club meeting. They couldn't do that this year. So everything had to happen online. So our enrollment system is all online, which is a little bit new. Um. So we feel like we've been doing a lot hand holding, a lot of education about that. Also, it's such a weird year and we really wanna keep kids connected to $4 \mathrm{H}$. Um, and so there's just been a much bigger push. Ya know, I'm promoting 4H so much more than I normally would. And we have this drivethru event so sign up. We are giving gifts away to join $4 \mathrm{H}$. Um, so it's hard to pinpoint it down to one exact thing, but we've just done so much more than we normally have. And we still have a lower enrollment. So it's not like all that work has shown us great numbers. Um. But we have.. We've gained new members that are not traditional 4Hers. 
Madi: So you've kind of seen.. You said that you've seen a decrease with the older members, and do you think it's because their version of what traditional $4 \mathrm{H}$ was and since it's different they're kind of like "Eh well, ya know, it's not the same" um kind of attitude with that.

Sarah: I really think so. I mean, it's not unusual, at least in Wetzel County, for us. As kids get into High School they move more towards FFA and not 4H anymore. Um. So they just don't participate at the same level. But yeah, absolutely. We didn't have a normal camp. Our fair was canceled. They couldn't show their animals. So those big traditional 4H things that really keep kids connected to the program. And ya know, they just love camp so much and just that love of camp I think carries them through the whole year. Um. And really for kids who are involved in livestock, I think it's the same way for the fair. Um. Ya know. That week is so much fun for them. And it keeps them engaged all year long. And like they put up with the other meetings throughout the year just so that they can do camp or do the fair. We didn't have that so what's, ya know. Plus they're trying to do school online. Like maybe they're just not interested in doing another online thing.

Madi: yeah, that's a lot (laughs).

Sarah: Yeah, and I don't blame them. I mean, I'm.. I'm sick of doing online stuff too but uh, ya know, that's really the only option we've got at this point.

Madi: Yeah, Um. So, what do you hope that your clientele is able to gain through your efforts of utilizing social media? Like what's the main thing you want your clientele to gain from your posts and your interactions on social media?

Sarah: Well I think it's two fold. Directly for our clients who are engaged in our program, it is a tool to get them information and to keep them engaged. So to keep parents involved and keep kids involved, especially the ones who are already there. And just sharing that information. Um, on the other side of it I want the public to know specially what Wetzel County $4 \mathrm{H}$ is doing. And so it is a tool to promote like "hey this is the work that Extension is doing in Wetzel County, here's what we are bringing to the community." Um, ya know. And really share like the "here are the reasons that you should continue to support Extension and 4H in Wetzel County." because we are continuing to do programming even during COVID. Um and you know, prior to the pandemic, we have had good support in our community, I just want to keep that at the front of everybody's mind. And that like "we're doing good work. We're doing work" Um. Ya know, your tax dollars are going to this program and here's some of the outcomes. Um. That it's not just um. Ya know, it's more than just cows at the fair I guess it really what it comes down to (laughs). We're teaching leadership skills to kids. We're doing STEM. Programming. Ya know, they're getting more out of it. And so "please continue to give us the funding" (laughs).

Madi: Yup (laughs). Okay. And how do you think that social media usage for work purposes is changing? And how can you adapt to this?

Sarah: (pause to think) Um.. I mean, for work purposes you have to. I feel like you just have to have a social media account. But I think I struggle with it personally because there have been times. You know especially this year that social media can get really nasty and just kind of be a 
place where it doesn't feel good to be there sometimes. And so personally, I would like to step away from it personally. But then I think well no, I have all these accounts for work like I have to be on Facebook and I have to be engaged with people and posting. Um. It's also like it is the way that so many people communicate with me. So people will message me like on my personal page if they have $4 \mathrm{H}$ questions or they have Extension questions. So I just, in that regards it's very hard to separate life from work because they can message you at any time. Um. And expect, ya know, I think we expect immediate responses. So it doesn't matter than a volunteer messages me on a Sunday night. Like, they want the answer right then like "no, i'm not really, i'm not on work time." (laughs). Um so that's uh, i mean, personally, for that kind of balance is a challenge with social media. I feel like I got off track with that question. I don't know if I answered it for you. (laughs).

Madi: Yeah, no. That was really good. That's a really good point. I have noticed that like social media, this year specifically with everything going on, has definitely made me want to take a step back too. Like I don't even get on Twitter anymore (laughs). There's too much going on, but, you know, when you're managing accounts like that, you really don't have the option to step away from it. But on the positive side, you are able to flood Facebook with positive things. Um. Instead of all the negatives, so.

Sarah: Yeah, I mean, that is true. When I'm on those accounts, it is not.. I don't feel like it's an ugly place to be when I'm in like the $4 \mathrm{H}$ world of social media. That's fine. It's the.. For me it's hard to kind of split those two and like "well if i'm on facebook for work, I get sucked into like the personal side of it" It's very hard to have self control and to not do that.

Madi: Yeah. And this one's kinda similar too. We kind of touched on it a little bit. But can you describe any challenges that you foresee needings to overcome in order to continue utilizing social media in the future?

Sarah: Um, ya know, I guess I. I do. We've talked about child protection issues. That's always somethings that's on our minds as far as - I want to use images of kids because that's how we promote our program. And like I said, those are the popular posts. When they see their friends or when they see their grandkids. Ya know, those posts get all the likes. So we wanna do that. I do.. I do worry if we are.. Kids who get shared by me on social media, they've all signed media releases. So like I have permission to do that. But I could see those rules um tightening up overtime. I think also accessibility. I guess I don't personally have a barrier to using any social media. But for people who do, we wanna make sure that what we're putting out there is accessible for everybody. And I don't always have the skills or personally think through like "how do i need to make this post so that anybody can access it and can get the information that they need from this post. And um, ya know, Facebook and Instagram have some of those tools just built in so I don't have to think about it. But there's also times that I know that what I'm putting out there might be challenging and I don't have.. I either don't work around it or I don't put the time in to make it as exceptional as I believe it could be. So I - I guess I see the future as either being a goal for myself or being a goal for Extension in general. Like how do we make sure we're reaching all our audiences? 
Madi: So you kind of just talked about this a little bit with talking about the guidelines um for not being able to post pictures of certain students or having to get release forms. Are there any other guidelines that you have to work with? um besides that.

Sarah: Um there are some. WVU Extension puts out kind of some best practices. Ya know, we do our best to follow those. Um. One guideline is that they-so i'm not the- necessarily like the owner of those accounts. Um. There's always somebody who is from Morgantown, from WVU Extension communications specifically, that is an admin on the account. And it doesn't mean they are going in and posting things, it just means, I mean especially if I were to change careers or change positions. They don't want somebody who's not an employee to be able to post on those accounts.

Madi: Yeah

Sarah: So they have control over those. Um, which is sometimes helpful because we run into issues and it's like "oh I don't know how to do this thing that's on Facebook" And they are the experts at it so they can go in and fix things. Um. There was one instance where I was in the midst of camp and having an issue. And they could- I could call somebody in Morgantown and they had access so they could post as that account. And share the information that needed to be shared. So, I mean, there are advantages to it as well. That's kind of the main one that we just have to have somebody from Morgantown on the account as well.

Madi: Do you foresee with the rise of using social media for Extension.. Do you see or kind of foresee them maybe establishing set guidelines or having more rules to follow, more hoops to jump through. Do you kind of see anything like that possibly being in existence soon?

Sarah: Yeah, I think so. Um. We do have wonderful communications department. Um. You know the University too. There do tend to be a lot of procedures and policies that happen. And so, I mean, they monitor those accounts and they want their message to be communicated in a certain way. Um. I don't foresee them like taking over and only allowing us to post what they say we can. But, I think they are very cognizant of "we represent the university" and so you know, there are certain ways they want that done. Um, yeah, so i think there could be more formal that go into place for sure.

Madi: Okay. (clears throat) Sorry, I'm like losing my voice a little bit. Um. But, would you consider yourself an innovator? And when I use the term innovator I'm thinking of like Rogers Diffusion of Innovations Theory. Um. So innovativeness would be how quickly you are to try new things and be one of the first ones, for this example, to use social media. Do you consider yourself an innovator when it comes to social media practices and why?

Sarah: Uhhh (unsure about herself) I don't know (under breath) I don't think I'm the first, but I'm pretty early. It doesn't seem innovative now, but I was one of the first people using Instagram for - as an Extension office in West Virginia, more specifically for $4 \mathrm{H}$. So that, that happened, I mean, I think WVU had an account and - but I had a - I had a 4H - I think we had a the county Extension Instagram before the state $4 \mathrm{H}$ even did. So that, I mean, that was early. But again, that was 4 years ago so it was like innovative then. I don't know that like I've really kept 
up with that. Ya know, we're not - I'm not on twitter. I'm not doing anything there. There's been some talk of like snapchat or um TikTok at this point and like I'm not going there. I'm just not (laughs). Um. Honestly, and I think part of that could be I'm getting older and so like I'm not the young agent anymore. And so like the young agents can- they're more comfortable with it and they can do that. Um (laughs) I'm the middle aged lady now. I'm not gonna take up those new things. Yeah, I mean, I guess I like what I'm already using. I'm pretty comfortable with it so that probably makes me not an innovator.

Madi: Well I do - I do think that it would be very nerve wracking to take over an account like Snapchat or TikTok. At least from my perspective, you can't really control those as much as you control Facebook and Instagram. So I think that if $i$ was in your shoes, I would be very nervous about utilizing something like that just because there's not as much control with being able to um use those accounts as there is with Facebook.

Sarah: Yeah, and you know, it's - this is not - my job is not full time communications. So I'm trying to do all this other programming too so it is a tool that I use to get information out about the programs. But there are people at the university and um, ya know, the state Extension office. But that is their full time job is to do social media. So that's great. Like they can do snapchat. They can do - they can try those new things. Um. I do always appreciate when they create the content and get it out and then I can just post it to our channels. I mean, that saves me a huge amount of time. Then I'm not worrying about what photo do I use and who do I tag in this. And you know, doing it all the right way. So, ya know, I always kind of keep that in the back of my mind. I'm not - I'm not the director of communications (laughs). So like- let somebody else handle that.

Madi: yeah. So thinking about other Extension agents throughout the state, can you describe of a time when you noticed maybe a different Extension agent utilizing innovative social media practice where you thought "hey I should do that" or "hey that's really cool" Um, can you think of an example of when that happened?

Sarah: Um. I don't know if you know Julie Tritts. She is the 4H agent in Wayne County. And she's assistant director for West Virginia $4 \mathrm{H}$ also. She has done really wonderful things with their Facebook. And specifically around promoting camp. When uh- back when we had camp in person, and it was probably a couple year ago, but they did some really great posts leading up to camp, trying to get kids to register for camp. Um. Incentives on social media. And, ya know, she was somebody I talked about Facebook with a lot because I was using it. But- I guess- and I don't really remember specifically beyond that it was around camp. But I remember talking with her about that. And she, from the Extension side of it, she really uses those analytics that you get from social media um as an evaluation tool. And so she's kind of taken it to that level beyond just using it to communicate but what kind of information you're gaining from all that data that you can find out of Facebook. Um. And so shes- she's looked at that and kind of come up with some great evaluation tools through social media.

Madi: And has she um - using those evaluations, has she shared that with you and other Extension agents so you guys have kind of been able to learn from each other in that aspect. 
Sarah: Yeah, she and I um we worked together on a presentation and I kind of talked about that Instagram side of things. And then she focused on how she was using her Facebook analytics in her evaluation reports. It got very basic of like "Here's where you click to find what you're looking for" But then how she was presenting that in some of her reports. So yeah, she's definitely shared that out with other agents.

Madi: Awesome. And that's it for my questions but if you have anything you wanna add that I didn't cover or anything you want to share with me. Or if you just have any questions in general for me about my research or anything. I'm open to that now.

Sarah: Okay, um. I can't think of anything else I really want to share. Um. I would love to find out more about your research or like even just some of the tips that you were giving me as we went. Like that was very helpful. Even some of those things like Facebook live. Like oh I've done it but I don't think to do it all the time.

Madi: Yeah

Sarah: Again, it's- actually Julie and I talked a lot about, you know, can you schedule posts. And I don't, I'm not great at scheduling. And I'm very much like on the fly. When I think of things I'll post it. Um. But kinda using some of those tools that I know are out there to kind of schedule and manage. So, yeah, I mean, when you're done with your research, I hope you're gonna share this specifically with West Virginia Extension.

Madi: Yeah. That is $100 \%$ the goal. So you guys can learn from each other and see what you all have been doing and kind of implement it more in the future. So that is the purpose of my research. So I'm excited to share that in May when I graduate.

Sarah: yeah, that would be wonderful. There's - If I can find the email, I will send it to you. We are doing a - we had like a professional development day in November and it was just kind of a day of different zooms and just agent sharing with each other. Ya know, what tools they are using right now. What programs that they are doing. But I would think what you are - some of that may be kind of basic to you but you could share that with agents right now, even before you are formally done with your research because it's very useful.

Mad:; Yeah. That's awesome. Yeah, if you find the information about that, you'll have to share it with me.

Sarah: Yeah, i would be happy to. Do you have many other interviews scheduled?

Madi: Yeah, so kind of what I've done is I've started, based on the survey results, which I had like 52\% response rates, which is like (clicks) awesome! So thank you for replying. I scheduled six interviews in the next 3 weeks. And I'm kind of getting more information and looking at things so I might schedule more after that. But that's where I'm starting to kind of find some spotlight people to look at. It's kind of a case study so that's where I'm at right now. So thanks for participating. That was super helpful. Super awesome. 
Sarah: yeah! Absolutely. Who's your helper back there.

Madi: Oh yeah, this is Riley

Sarah: Hi Riley

Madi: She's a black lab. She's a sweetheart. (giggles)

Sarah: I love labs. You see ours back here. They're good companions right now.

Madi: They're awesome, yeah.

Sarah: Alright, well thank you Madi

Madi: yes, thanks very much. If you have anything else later that you'd like to add or any questions or anything for me, just feel free to reach out whenever. You have my email and my phone number is in the emails too so feel free to reach out in any way. I'm always available.

Sarah: Okay. Yeah. And I would say, same. If you have something that comes up that you need more quotes on or something, just let me know.

Madi: Awesome

Sarah: And $i$ think in my signature there are links to both of my work social media pages if you need to see those. I don't know if you are looking specifically at accounts or not, but, they're there.

Madi: Alright. Well, thank you very much. Enjoy the rest of your day

Sarah: You're welcome. 


\section{Elizabeth}

Madi: Perfect, all right yeah so I'm just going to ask you some questions, and we'll just go from there. Should be really relaxed and fun.

Elizabeth: Okay, sounds good. Okay.

Madi: So, can you describe what you would consider to be beneficial social media practices that you implement for work purposes?

Elizabeth: Um, so this year we have really found out the benefits of social media. Um. We've done a lot of virtual teaching through, over Zoom, through teams. And the best way to get the word out there for different events is through social media. Um. As you probably know, we had virtual camp, and we had, you know, we've been teaching classes all summer and into the fall. And I got kids who aren't normally $4 \mathrm{H}$ members to sign up. We had kids from my community that saw my post on Facebook, they followed the extension Facebook page, and they're like "oh that sounds really interesting, but I'm not in 4H." I'm like, "That's fine." We actually had recruitment kind of go up this year because of folks seeing all of our stuff on social media, so that has been very helpful.

Madi: And that's awesome and I know that when I was speaking with another individual she had mentioned that she saw a huge increase in new members, specifically younger members because of using social media, but she did see a decrease with possibly the older members who were used to the traditional methods of um $4 \mathrm{H}$ specifically. Now, did you see this kind of same pattern, or was your experience kind of different?

Elizabeth: Yeah, um like I said, we got a lot of new members like out of the community and those were some of like the clover bud age kids, so there's kids that are five to eight years old. And then, a lot of my older kids. This.. at this time in their life we normally would lose some of them to FFA, regardless, because of their in it show animals. They liked the camping experience, they liked all that stuff so we did lose some of them, because everything was virtual and we didn't have hands on experiences. So, I saw that as well. I saw the major people who participated were kids who were like, 8 to 13. That was our age range that we were really recruiting with, and that was basically because their parents saw it on social media, and wanted them to participate.

Madi: Okay, that makes sense. And you also have an Instagram account. Correct? (Elizabeth: nods) Okay. And when you're using Instagram do you typically gear it towards the younger kids and then Facebook towards towards your parents or do you think it's kind of all parents are all ages like um.. Does that kind of change depending on what you're using?

Elizabeth: So with Instagram.I kind of make it more of a extension office wide type thing, so anything that we're doing a lot of times it does seem like it's more for each focused, but I tried to share out stuff on there that is countywide, or you know, our whole office can use it. If I'm targeting younger kids on Instagram, I'll probably try to put stuff on like the Instagram stories. 
Elizabeth: Um They seem to, I don't know if it's they know to look at those and parents don't know to, you know, the older generation doesn't know how to use Instagram stories and that type of stuff. Um. But it's kind of an all age type platform to use.

Madi: Okay. And when you, um.. when is your clientele most engaged with your social social media posts and how can you tell?

Elizabeth: Um, so if I post something like in the middle of the day I'm here in the office working on something and I will say, I need to get this information out, it's usually not until five or six o'clock in the evening until people start responding back to the post or liking the post. It's usually the evening time once people get home from work, they are scrolling through Facebook or Twitter or my own personal Twitter or um Instagram, and they see our information, that's when they'll usually respond back or they'll respond to an email or they'll send me an email about something they saw on Facebook. So yeah, it's usually in the evenings whenever we get the most response back when stuff after office hours.

Madi: Okay. And you mentioned a personal Twitter, do you typically use that for some work purposes to?

Elizabeth: So I will share, like I follow the extension page WVU extension Twitter page. And so, if our Dean put something out on there I'll like it or I'll retweet it. I usually don't put out any recruitment stuff on Twitter just because I don't have a lot of kids on my Twitter, my personal Twitter. It's a private account. Um, but yeah, I'll share just basic stuff on there.

Madi: Okay, that makes sense. Also, what are some key examples of times that you utilized social media to remain connected with clientele as a response to the quarantine protocol from covid 19.

Elizabeth: Um, so, this past summer we did a foods program for families in our county and it was um beyond our normal reach of for $4 \mathrm{H}$ youth, so it wasn't just $4 \mathrm{H}$ families that were qualified for this, it was any low income family that applied. and the information came out for this right after all the school shut down. So I didn't have any way to get information out to families through this whole system, other than like I ask teachers to, like, send it out in their emails weekly or whatever. But I would say $90 \%$ of my responses came from a Facebook post asking if people will be interested in a CSA box, type program. And that's, we got a lot of outreach from that, that it was folks that we normally wouldn't have reached before. And then this program was derived from, you know, reaching families during covid and making sure they had their necessities met.

Madi: And kind of on the opposite of that, what was something that you did that you thought would go really well, but maybe didn't.

Elizabeth: (laughs a little) Um, so when we were first sent to work from home, I was putting out daily posts, I went in and I scheduled them on Facebook through our extension page. And it was just educational pieces people could learn from, ya know their podcasts they could listen to scavenger hunts they could do at home. No one ever reacted to them. They were just kind of there. Now, if folks did it, I don't know, they didn't like it and post it back and say "hey we really 
like this." They didn't really react with the posts, no one ever really liked them other than my mom. But, uh, yeah those posts I thought they would do a lot better. I thought you know we could, I could get some service and teaching out of that. And then I said no one really reacted back to them, I found them beneficial. I learned stuff from and post but there was just not a good community feedback from that.

Madi: And do you like... can you think of a reason why maybe you didn't receive the responses that you would hope for?

Elizabeth: Um, I don't know if it just wasn't like topics that people were interested in, or if it was just such an overwhelming time, like I was providing some additional educational resources, and people were trying to figure out how to do this virtual learning school from home. If they were going to homeschool program. Like,I think it was just a crazy time for folks. And they were trying to balance everything.

Madi: Yeah. And have you gone back um since then and like posted similar things and have they done better?

Elizabeth: Um, I don't post this often but when I run across stuff. I will try to post it out there and it seems like um we have had a little bit better response but I mean, nothing major, not a huge increase. It just seemed, we did have.. our extension page did have an increase in likes over covid times. We were posting out, like in the summertime, we posted: "How to can stuff from your garden" and "how to, you know, harvest- when to harvest your plants" and that type of stuff. And it seemed to do better then, that was stuff that was more relevant to the time and type of thing as well.

Madi: Umm.. based on your survey response, responses, we've talked about Facebook and we've talked about Instagram, and I know we touched a little bit on this. Are there any other platforms, social media platforms that you use that I'm missing?

Elizabeth: Um, not for work purposes, um, they, they kind of don't want us to use Snapchat for work purposes, just because it's such an unknown of what's happening to your stuff, you know.

Madi: Yeah, for sure.

Elizabeth: So they kind of tell they tell us not to use that, Instagram and Facebook are the two ones that they push out the most for us to try to promote events on.

Madi: Okay, and we touched on this a little bit but to go a little bit more in detail. Um, can you describe a little bit more um.. what you would use different platforms for, so what would you use Facebook for, compared to what you would use Instagram for.

Elizabeth: Um, Facebook is just kind of my catch all like I, I tried to push, push most of our information out on Facebook, and um to reach a large, a larger crowd of people on Facebook that. I felt on Facebook, we can reach the grandparents, we can still reach the kids for some of the kids that are still using Facebook. Every generation is kind of on there. We always try to 
provide something for each of them. And it's more of a recruitment tool, I feel like than instagram. Instagram, I just put out like, you know, Snapchat, not a snapchat, a snapshot of something we're doing like a camp or a state fair and it's just like, it's not really a promotional piece but it's more like what we're doing type piece, if that makes sense.

Madi: Yeah. And also, when you're.. so like on Instagram obviously, most of the time you're posting pictures, since that's like such a photo generated app. Are you also doing the same on Facebook or do you think Facebook is more like words compared to pictures or videos or something of that sort?

Elizabeth: Um, it depends on the event so like, normally when we have camp, I'll post pictures all throughout the week. And that's, I mean we're pushing more than one picture at a time and I know on Instagram, you can post more than one picture. Um, but usually from Camp we just dump an album of photos there. And we'll do a slideshow at the end of the week. It's easier to post a slideshow on to Facebook than it is to Instagram, to share it that way. I do have our accounts linked. So, when I post something on Instagram, it'll go to our extension Facebook page as well. So sometimes it gets, you know, they get put both places.

Madi: Okay. And you did touch on this a lot, but one of the big things that I've been finding in my research is that utilizing social media and extension specifically can really reach new audiences, potentially individuals who are unfamiliar with extension which you nailed earlier you pretty much said exactly what I'm getting at, but can you share a specific experience that supports this claim?

Elizabeth: Um, yeah so a lot of times we have events that are just, we just do them one time a year and people kind of forget that we do stuff like that, like, we will do pressure canner testing, and that's usually something that's just done in the summertime when people were using that. And this year, I didn't get it put it in the local newspaper, and you got a lot of older folks they depend on that newspaper to get their news, but I have tested more pressure canner lids, this year and I only put it out on Facebook. I don't know if that - that was the cause of the influx or if it was, everyone's canning now because of the pandemic. I don't know, but it does seem that we are reaching different folks that we normally don't have contact with.

Madi: And have you really done anything, like you're reaching these new folks, have you done anything specifically.. Have you changed anything from compared to before covid to now, in order to reach these new folks. do you think that you've made any changes specifically?

Elizabeth: Maybe just the way we approach, the way we post information. I know a lot of times before, you would just throw up a picture and you think people know what you're talking about. Now we add a little bit more of an explanation of what's going on, how to contact people, what's the best way to get the information to the extension office. And just adding more information and giving people more detail, I feel like gets them more involved as well.

Madi: Okay. And what do you hope that your clientele gains through your efforts utilizing social media? 
Elizabeth: Um, I feel like they just need to know more about what extension can do for the communities. We try to post a wide variety of, you know, what our ag agent is doing and what the $4 \mathrm{H}$ program is doing. But it's not just what $4 \mathrm{H}$ is doing and what the ag agents doing, it's more of different services we can provide such as soil testing, and like I said, pressure canner testing and, you know, how to get the kids involved, how to know about the dinner meetings that we normally hosts for farmers, but we, you know, it's information that I'm more than just farmers and 4Hers need to know about. They tell us all the time, we're the front door to WVU, we're, you know, we're in every county, we're 55 counties across the state. I hope they, you know, gain some knowledge of what extension can provide for everyone in the county.

Madi: Do you think that you guys have done a pretty good job at that in your county, like have you been pretty successful being able to display that message?

Elizabeth: Um, I feel like we've done better since the pandemic because we've been trying to, you know, we're just so set in our ways of what we normally do every year but this year we weren't allowed to, or we weren't not allowed, but we just weren't able to do a lot of the stuff that we normally do because you know, covid. So I think that we've been able to broaden our range a little bit. We've had more folks call the office and just ask questions. People who didn't know what we did before, now are some of our regulars that call us all the time.

Madi: Do you guys have any workshops or anything specifically on like social media usage that either extension provides for you or other agents like. Is there anything like that that's been going on because of covid?

Elizabeth: Um, we did have, with our CEOs conference. Um kind of like a zoom type, like how to use zoom training. We've also.. we've also done that with our $4 \mathrm{H}$ volunteers because a lot of them are going, they're posting their meetings virtually now. And we had a tough time in the beginning, trying to get them to do stuff virtually, I think it was just, you know, anxiety of using a computer, stuff like that. So we have provided some trainings throughout the Potomac Collins and Eastern panhandle region. We've... all the agents have kind of gotten together and provided that type of training to help leaders become more comfortable with using technology.

Madi: And do you think that it has for the most part it's pretty much worked like the workshops and getting them encouraged like, do you think that there's still little bit, like a little bit of,I would say like a bridge between your club leaders and your group leaders and the use of zoom and social media and stuff. Do you think that they're pretty comfortable with that now?

Elizabeth: Um, locally. We haven't had much success with getting leaders to do virtual stuff. they're kind of stuck in the mindset of the kids. Well, it's not really a mindset, the kids are burnt out once they're done with their virtual schooling and they want to be outside doing something. I would say about half of my clubs have at least attempted some sort of virtual meeting, I did. I've done virtual leader, officers training school and that type of stuff. And actually we just finished a series across the Potomac Collins and Eastern panhandle with how to use zoom and Padlet and different trainings. and we're waiting on evaluations to come back on that, to see if there has been an improvement in any of that area. 
Madi: And are your.. are your clubs also using social media. like your clubs have Facebook pages or Instagram accounts, or they kind of just on their personal accounts for that?

Elizabeth: Um. They, they mainly have a Facebook group for their clubs, I know, three of my five clubs have a Facebook page that I can go in and post in or the leaders can post in. And then I have just a separate private group that is for any $4 \mathrm{H}$ member in Pendleton county and their families. So it's stuff that not necessarily needs to go out on the Pendleton county Extension page for the whole world to see, but it's like information like hey you're.. the photography contest stuff is due in March, like this basic stuff like that, that normally would come from a club leader if they have a regular meeting but we haven't been having regular meetings, so that's kind of how I get that information out as well.

Madi: And how, how do you think that social media usage for work purposes specifically. How do you think that social media usage is changing? And how can you adapt to this?

Elizabeth: Um, so it is definitely changing. extension as a whole has really upped their game with using social media to promote kids for our virtual classes. and it's just becoming kind of the new norm. Um, I actively taught classes from, I think we started in April, up until about two weeks ago, we've, we've taught classes, different topics. But I think it's just becoming the new norm. if you want to get the word out there you, you've got to put it on Facebook, I mean everyone reads Facebook.

Madi: It's very true. And do you have.. So thinking of like any kind of parents that are kids even that aren't on Facebook. I'm assuming you still have your other means for communication like do you replicate it like send out mass emails with the same information that you're posting on Facebook. How do you do that kind of stuff?

Elizabeth: Um, I have some parents that I know they're own Facebook. Um, but they won't provide me with an email, which is kind of weird. And so we, just to get around that, you know, so they can say "oh I didn't see it on Facebook." Um, we send out postcards with information. They go you know, by snail mail. And then every once in a while we'll put out a newsletter if there's a ton of information or if it's like time for camp with all the enrollment stuff, everybody gets their stuff through the regular mail as well.

Madi: Okay. And can you describe any challenges that you foresee needing to overcome in order to continue utilizing social media?

Elizabeth: Um, just become more accessible for. They preach to us all the time about being ADA compliant. And I know a lot of people still struggle with that, and it's because you have folks who use screen readers and stuff like that um to read what's on their iPads or on their computers. So I would say that's probably the biggest challenge and it's not a new challenge, but it's been.. it's been a challenge for a while. But yeah, just being compliant, so everyone can see what you're posting. 
Madi: And do you think that you guys have done a pretty good job at being able to do that, or as to the best of your knowledge. I mean that's obviously a really hard thing to accomplish. But have you been doing that and trying to be able to do that with all your posts and interactions?

Elizabeth: um, for the most part, yeah and they've provided us with some trainings that help with that. So they tell us "Don't just post a picture on Facebook." You have to put exactly what's on the photo in the caption as well. So if I'm posting, we're having this tray.. a picture that's like a PDF or a picture that has words on it. All the words that are on the picture should be going into the caption as well. So people with screen readers can read the words because they, it won't pick up the words on a photo.

Madi: Okay.

Elizabeth: So, yeah, I think we've been doing somewhat better with that.

Madi: Yeah, that's.. that's one thing that I personally hadn't thought of so that's really good to know to post captions with the pictures, that's very cool. And can you describe how you work within guidelines for using social media. so any guidelines that Extension has given you or anything like that.

Elizabeth: Um one of the big guidelines they tell us is to just make sure that.. We've had a big training on it right at the beginning of the pandemic, but watch what you're posting on your personal page. To begin with, they were telling us you know don't add your $4 \mathrm{H}$ members or your volunteers on the personal page. But, I mean, in a small community that's really hard, like, you know how it is.

Madi: Yeah, absolutely.

Elizabeth: Now there is kind of their big guidelines are 1. to just be careful what you're posting and 2. to make sure you're compliant with the ADA compliant stuff. Um. Other than that, there's not really, I mean, there's not too much more. It's pretty simple.

Madi: Mm hmm. Yeah. Now do you think that maybe there's going to be more guidelines in the future? Do you think that that might be something that comes out as a response to the heavy, like the heavy push in social media, because of the pandemic pandemic and everything going virtual, Do you think that there might be new guidelines to come out in more like rules and regulations to kind of follow? Do you foresee that being a thing?

Elizabeth: There very well may be. I mean, there seems like all the time there's new guidelines whether it comes from extension or if it comes from Facebook or whoever, I mean there's always new guidelines that you're going to have to follow to, you know, make sure you're, you're doing it correctly to get your word out there but doing it in a proper way.

Madi: For sure. Also, do you consider yourself to be an innovator in regards to social media practices. And when I'm using the term innovator, I'm thinking of innovativeness based on Rogers definition and his Diffusion of Innovations. So are you one of.. do you think you're 
maybe one of the first people to be willing to try new things on social media and put things out there and use it? And has this become predominantly true with covid and the response to covid?

Elizabeth: I would say I was pretty active on our Facebook page, like to begin with. And so I was pretty familiar with it so trying new things, I mean, you can always try it once and if it doesn't work or if you don't like it, you don't have to do it again. Um, so yeah I mean, there's, they're always coming out with something new for us to try to reach our, our clientele a little bit better. Um, like I said, I'll put it out there. I'll try. If it doesn't work, we try something else.

Madi: And do you think that I'm, like, thinking about your other extension agents obviously you guys are close and talk amongst yourself. Do you guys typically, share ideas with each other and share posts with each other, those kind of things?

Elizabeth: Yeah, so usually if we look if I see another agent post something or another extension page post something. I'm just out of general kindness, will you know, ask "you mind if I share this?" One good thing we do have across our region is we meet monthly to discuss different things we're doing and we work together a lot, which I mean, I feel like it's been even more since covid started. So, we have a lot of the same posts, we're doing a lot of the same things.

Madi: Which honestly probably helps a lot because if you think, each county especially in your region, you know, you're all posting similar things, doing things at similar times. And it's less work for each one of us specifically if, you know, somebody does this month or this week or this assignment and then you all can share the same.

Elizabeth: Yeah. Yep.

Madi: And kind of bouncing off that can you think of a specific example or time that you noticed another agent throughout the state anywhere that did something that was innovative, that really made you think, "Oh, I want to do that" or "that's really cool" or, "I wish I could do that in my county." Can you think of like, a specific example of something that somebody did?

Elizabeth: In July, or probably closer to August or September, um, some of the folks in Mon County, put together these buttons that had the teachers' faces on them. I don't know if you saw those or not, but it started in Mon county and it kind of just spread like wildfire and the folks who had resources across the state went ahead and made buttons for folks in their county. I ended up with like 15, but that's a typical response for Pendleton County, so it wasn't really surprised. But across the state, I think we ended up with close to 1000 buttons being distributed.

Madi: That's really cool. And I did, I did see those posts back when they took off. I didn't realize where they had originated, in Mon county, but that's very cool. And that's, that's pretty much all the questions that I have for you. I got a lot of awesome information, but if you have any questions for me or if there's anything that you would like to add that I maybe didn't cover, any beneficial information for my research. I'm all open to that now.

Elizabeth: So, when you're talking about evaluations and the trainings and stuff for using social media. Once we get our evaluations back, right now folks are still taking them, we're hoping to 
have him back like next week, because we have to have them for our files. Um, I would be happy to share that information with you over how folks enjoyed or didn't enjoy or whatever with the zoom and Padlet and social media trainings that we did because I feel like that would be beneficial for you as well.

Madi: Yes, that would.. that would be awesome.

Elizabeth: I mean it just covers, it just covers the eastern panhandle and the Potomac Collins, but that's, I don't know but Jefferson, Morgan, and Berkeley had a big participation in that as well. Yeah, so I feel like, and they're always ones to fill out surveys for us when we do stuff. So we probably have a good chunk of data that I mean if you can use it great. If not, you can throw it out, it doesn't matter but I'll share with you.

Madi: Yes, thank you. I really appreciate that. And you, you have my email and my phone numbers in the emails too, so feel free to reach out with anything else as well. Do you have any other questions, concerns, information?

Elizabeth: Not that I can think of right now. If you need anything else he didn't help you know next semester, feel free to reach out, I'm more than willing to help out.

Madi: Well thank you very much. I really appreciate it. 


\section{James}

Madi: All right, well, just to make sure. Are you okay with me recording this?

James: Oh yeah. Yeah.

Madi: All right, I'm going to go ahead and start the recording. And we can jump right into things.

James: Perfect.

Madi: And I know we've talked a lot about my research but before we start, do you have any more questions about what I'm doing and stuff like that?

James: No I don't think so. I think it, I think it'll be interesting to see how it correlates between a pandemic year versus what I would classify as a normal year so.

Madi: Yeah, which there's tons of research on it in general so will be easy to compare those things. Well, my first question is, can you describe what you would consider to be beneficial social media practices that you implement for work purposes?

James: Um I think the most beneficial is just keeping the general public up to date, as far as sharing when tag in is or when we have activities or projects ready for pickup. We've sent just plain emails, we've sent postcards we've, you know, every other option, but in a digital age that we're now in social media tends to get the most publicity, as well as the most feedback.

Madi: Perfect. And when you're posting things are there certain times that you think your clientele is more engaged with what you post versus when like they're not as engaged?

James: Absolutely. So we've actually looked at this as far as I mean Facebook specific, you can go in and see when you get the most hits.Um. So we always try to get all of our published stuff out right around 10:30, because we found that most working adults check their phone somewhere between the hours of 11 and 1. And that's when they'll see most notifications as well as emails. So, and that's something that we've really kind of. We started to notice it early on at the end of 2019, and then for 2020 it really started taking effect. We started sending a lot more stuff out, you know, not necessarily in the evenings. So we either aim for that 10.. We like to post at 10:30 so we get that 11 to 1 , or we'll wait and actually post it after $10 \mathrm{pm}$, because then it shows up in their morning cycle as they're going through and getting ready.

Madi: Okay. And do you think that that has changed at all because of covid, do you think that that's still true even though a lot of people are working from home?

James: Oh yeah, and the reason why is because if you look at when parents have time to sit down and actually you know take just a minute and scroll through. It's either going to be when they're feeding their kids lunch, or after they put their kids to bed or before they get their kids up in the morning. So that's why we kind of aim, you know, 11 to 1, somewhere in there you're probably 
going to feed your kid lunch. You know if you put the kid down at 8:30 or eight o'clock even, you're more likely to see at nine o'clock post than you are a seven o'clock post.

Madi: Yeah. And also, so when you're.. I'm like thinking of these posts that you're making. Are there certain types of posts or things that you're posting that are drawing more attention than other posts?

James: Anything that has... So we try to stay away from pictures that have text on them because with ADA you have to of course include all text that's on a picture in the body of the actual post.

Madi: Yes.

James: So it's been.. For us some of it as easy as you know we we make it in publisher so we'll copy and paste verbatim the text or we'll make some type of, you know, find a really nice just picture that we're allowed to use either one that's taken is approved by extension or one that we have the rights to use will post it and just put the message out. Um. We tend to use the, you know. (pause) I think if you ask my secretary she would disagree with this. I think we get more hits from having the picture with text, because we always try to make them look a little bit better. Um. So we actually just posted a winter wonder camp box, and we were looking for 25 kids to sign up. We had 32 sign up in the first, I think it was six or seven days. And that was just members and then we have 16 or so, already signed up for clover buds, and we were hoping to max out at 24-25. But since we've already hit that we're actually reordering everything. We were (connection error).. the first one to get enough to cover the second wave. And we've only shared it like two or three times.

Madi: Wow and that's one that has text included?

James: Yes, so it's a, you know, it's a winter scene, a snowman. We make sure to put the flying WV. I think it's actually a snowflake. Um. But you know we make sure there's branding on there but we also want to make sure that if the kid does see it, the kid is the one that's interested.

Because, you know, parents might necessarily see the text and read through the text. But if we're trying to get kids appeal, they're going to notice a picture more than they will bunch of words.

Madi: True. So, when I'm like listening to what you're saying to me it just seems like the ones that you take the time to like think through and plan out and like make look more appealing are probably the ones that are getting the most hits in the most attention.

James: And I know that's not so we're like, kind of, we're asked to steer away from them unless we do abide by all the ADA stuff. So I think if you would ask kind of our comm director she would say oh no we post pictures and, you know, put text in the body of the thing. Um. While it works, I don't think it works as efficiently, because then what I've noticed and what I've, you know, heard a lot of volunteers say is they'll actually click on the picture and just save the picture.

Madi: So they're not saving the words with it? 
James: Yes. So, It's a lot easier for us to have that image, where they can click on it, and store it in in their phone, and they know the rules that they have to retype it and everything so a lot of them will save the image and then go through and add text to it for their own clubs or their own organizations or whatever they're in so

Madi: And can you think of some specific key examples of times that you did utilize social media as a way to stay connected, like any specific things that you all did?

James: Absolutely. So, Tri County camp, kind of got flipped on the end. So instead of just canceling, which some kind of did, or adopting kind of the statewide you camp you are linked. We kind of met the road in the middle. So we did Tri County virtual fundings. And essentially what we did was we sent out a complete box of activities with camp classes, assemblies, crafts, a little campfire pin, like all kinds of stuff that would remind the kids of camp or make it as camp esque as possible. And then they actually would log in for specific, for specific times. But it, we had 238 Kids sign up for it. And we had I believe right around 200 or, it was like 199 or 193 that were engaged every day. Yeah, and that included Zooms, that included some cabin meetings, that included council circle. So those are kind of very specific to the $4 \mathrm{H}$ program. But I think it is what kept our kids engaged, so they were posting pictures of, you know. Every day we had some type of challenge, every day they would post pictures of, "show me what you did in class once today." Sometimes it was as simple as putting, you know, a picture of Playdough up, but some kids were, you know, making you know 25 second videos and uploading. So it provided some really great content that we've since used to kind of want to impact kind of report analysis of camps. So I think being able to pull that stuff off, you know, social media does a great job of being able to create, you know, create those graphs for us or be able to create those documents that we don't necessarily have to, you know, sit down with Excel open and manually do over and over and over, it does it for us. But I would say that whole week was a success because of social media.

Madi: And did you use Padlet mostly for that, Correct?

James: Um, so for that we actually took Facebook, we actually created a hidden page and that became the most used. We did also have Padlet, but we did not have very many kids actually engage in Padlet. On the front burner of Padlet for us. So we didn't really know how to incorporate it as best we could. But outside of that we have used Padlet quite, quite a lot.

Madi: So you just had like a private facebook page that anybody that was registered for camp would be asked to like join the page and that's where they could share all of their.

James: Yeah so we sent a private link once they registered, so they could, you know if you, you know, if you want to put your child that I know you don't have. But anyway, if you want to enroll, I don't know, Tyler Quinn, into camp you are, whatever it is. So, whatever was your name as well as your emergency contact. Just in case someone wasn't on Facebook or, you know to accommodate those that want additional details. They could, so some of some of them were grandparents, some of them were first step kind of adults. It just kind of gave us that sense of feel and then we also, it was, it was kind of cool because we actually ended up having closing council circle that we could record that kids who couldn't be there on that zoom could watch later. And 
that was something that I really liked so we were actually be able, we actually were able to make an age out video. So, for the 21 year olds that couldn't claim honors at council circle. They were able to claim honors throughout camp in their favorite spot, record it. And we put it all into one video that was able to be shared. So it was, it was a bummer that we couldn't do it but by social media allowing us we were able to produce this video that we can you know share, regardless of what time it is.

Madi: And did you use like Facebook live at all on the Facebook, on the private Facebook group?

James: So we did not use Facebook live. Just because of everything happening, we wanted to make sure that just.. I mean. For example, one of the agents in particular, you know, facilitates it but does not have internet at her house. So she would be going back and forth so a lot of the in person stuff, a different person would facilitate, or we would post it. And you know she wouldn't get it at a certain time and a lot of what we do as such as a dynamic group. You know some of those videos that we used. We definitely learned a lot so I bought a gimbal because. (lost connection for a few seconds) So, which has been nice. So we recorded a video for the Pledge of Allegiance and the flag ceremony. So we brought a group of people out social distance, wore masks. They did flags, they did taps, and then we could share it throughout the week. So we ended up, you know, having a lot of kids. We would post the links on Facebook when it was time, they would click the link that would automatically bring them to zoom. So, and some of those had upwards of, you know, 200, you know, 175, 200 kids was our biggest. I don't think our lowest had less than 50. So it was able, you know for that instant, okay they need to look on Facebook to get the link and go. It wasn't like they didn't really have to wait a whole lot to get it into this. So it was really cool.

Madi: That's awesome. And I, I know that a lot of people did like different for the virtual camps and stuff but I think that the private Facebook group Facebook group is pretty good. But did you run into the problem at all where let's say that there was a camper that no like family members had Facebook at all or they didn't have Facebook like did you ever run into that?

James: We did and that's what we had the initial Padlet setup. We were very fortunate, we could either offer them through email or the Padlet. A lot of times they.. I'm trying to think, I think there were only 5 out of the 238 that did not have Facebook. And a lot of them were able to either have, again their adult or like a grandparent or whoever there was next on their emergency card. one of those people had it. So we didn't feel that it was a large kind of disconnection. We did have some that just wanted the email, which is perfectly fine. We literally would copy and paste from email to Facebook and vice versa.

Madi: All the same information, regardless?

James: Yup. So it kind of kept everybody on one page. It was nice to be able to facilitate that week and not have to worry about, you know, making sure to go around and send everybody's emails or go around and send everybody remind texts and such as.. We also had a large, I think we had 33 adult volunteers. So it gave us a large pool of moderators and collaborators to go in and check, you know, the three agents were in charge of admitting people into the page, nobody 
else had that ability. So, you know, we were able to keep it extremely secure. There was no ability to share outside of the group, unless they took a screenshot and then posted a screenshot. But ultimately, I think, you know, in the digital world in age I think if we were able to facilitate a great activity for positive youth development, but at the same time you know make sure that we had that high level of safety, that we want to, you know, ensure whether we're in person, or virtual.

Madi: Yeah, which is a huge, like a huge thing when hosting any kind of large event like that on social media is being able to make sure that everything's private and things aren't getting posted like pictures of somebody aren't getting posted elsewhere.

James: Exactly. So that was the kind of, I don't want to say that was kind of our trial run but that was kind of a gold deploying last slide. Because none of us had really tried to manage a project that big, just on social media. And it kind of gave us that push to kind of make that happen. One way or another, just because of. I mean, ultimately, because we had to, you know.. that, that's what we're known for that's our flagship program. So for us not to have some version of it was just not acceptable.

Madi: Yeah. And do you think that you know maybe in the future, hopefully we'll be back and being able to have in person camp soon, but do you think in the future that it could possibly be incorporated where individuals who can't attend camp, or are in and out of Camp during the week and things like that, you could potentially have it on social media for those that can't attend and kind of do like a, like a mixed thing?

James: Yeah, being able to do that mix would be fully possible. I think we'd have to make sure we had enough people sign up for it to make it worth it. Um just looking.. So all of the fiscal responsibilities came through our office. So it did cost. Oddly enough, it did cost about the same for an in person camp because instead of being able to be in person and buying food we were you know virtual, but we had to still buy the boxes and we had to buy all the materials individually. So it was kind of a weird. What we would normally spend you know \$5-10 on camp classes automatically which came like 20 to 30. Assemblies became that much more expensive and, you know, we try to stay around that hundred dollar mark. But we still gave the kids a T shirt. You know, we gave them all that, we gave them snacks for when we have meetings on the green. So they had snacks that they can enjoy during those meetings. But every night we logged on and, you know, we kind of closed off the day. And then we would post kind of our closing thoughts, and it you know it. To me, those are the magical moments of camp. So those are what we wanted to make sure we hit. council circle was great. You know, flag ceremonies are important. assemblies are great. but it's in there's true little kind of downtown, downtime not downtown, sorry, moments where kids really remember camp. And it like that's the craziest part but you know it's it that's the parts that build camp. It's not necessarily the overall ron rons, those kids of solum moments

Madi: Yeah, and being able to execute that on social media is a huge challenge but it sounds like it was pretty successful. So, that's awesome. 
James: And I don't know, well I can. So, the page is still active. So if you want to see it as well. I can add you to that group.

Madi: Yeah, that would be awesome. So I can go through and just kind of see some of the interactions and what was posted and what you're talking about.

James: Yeah, I know we have social media releases for every kid but two.

Madi: Okay.

James: But that way if you wanted something out of it I could of course, I couldn't tell you up front, but you could provide me a list and I could tell you yes or now about certain things.

Madi: Okay. Yep. Awesome. And thinking back.. so like moving away from Camp a little bit because I know we spent a lot of time talking about camp, but just any social media platform at all, what was something that you thought was going to go really well and then maybe didn't have the response that you anticipated?

James: Um, so I'm going to answer this kind of, I don't want to say poetically, but this is how I would write it in my file that I would have to submit. Given the circumstances of the year, anything above zero, I was glad to have. Um, you know, in a standard time where we're used to seeing, you know, hundreds of kids, you know, I try to go to every $4 \mathrm{H}$ meeting I can, try to go through at least, you know, two or three or four months. Um. So going from seeing up to you know 300 kids a month to not being able to see any, except for the virtual platform. I thought anytime that we were able to reach out um was a success. We haven't had any projects that have been a total flop. We typically see.. It's very interesting because we very rarely actually have the same pocket of kids come back over and over. And we've kind of done that on purpose. So we've offered some very stem based activities. Most recently, we announced our poster contest for the state, kind of guidelines, they send out and then we kind of bumped it up, wrote a grant, got some money for um kind of art kits, so we were able give any kid that signed up for the state poster contest got an art kind of kit. It was like \$2 a kid, but it was just kind of materials that they would use. We also have done a tie dye. We're making tie dye masks for first responders. So we put it on Facebook, we had funds, they were $\$ 6$ apiece. Kind of some just discretionary funds were able to about 25 packs and you know we put it up and literally within 10 minutes we probably had five kids register. So I don't think anything we've done with social media has been a failure. I think we have kind of built our members and parents, kind of in a, we kind of shaped them in a way that if they need to know information they can check a certain spot for it. So I mean, that has kind of that's what's really helped us, our parents know that this is the way they're going to get you know information from the club leader or from me. But it has ultimately kind of been a blessing to us to be able to get that kind of information out, because we can do it. What used to be in a newsletter or postcard and take five days to get to them they're now getting in a matter of seconds. So I know that kind of doesn't answer the question, but I don't think anything we've done has been a failure. Because everything we've done has been new. If I would have to pick. We've had some struggles when we first started posting. Just because, you know, we wouldn't necessarily make them flashy. I don't know what the, you know, magic word to call it is. But we figured out and that's what we, I think. And again, that's my opinion. But I think the 
posts with words, anything where we can make it look, you know, just a little bit of extra special to draw attention to it makes it better. And I mean, I, everyone is guilty of this, you would much rather look at a picture than you would read a paragraph. So we tend to be very short and brief for.. and we always put "for more information please check our website, you know, www.extension.com/berkeleycounty" We've been using the (newsweets) platform that kids use to register for $4 \mathrm{H}$ as well. It has a lot of social media type abilities so we can post an announcement section, we can send direct messages through the messaging, kind of, it kind of like an email but it's only through the (newsweets) platform. But I would still say Facebook is number one, how we reach out.

Madi: And when you're talking about, like when you posted about the face masks and you had five kids sign up instantly, is that typically older kids signing up for themselves or is it typically parents signing their kids up? Do you see a pattern in that or does it kind of just depend?

James: I would say it really depends. We actually, I would say most of them are parents sign up for kids. Even with our older members, but we have done. We have so many members that we actually limit ages to who can sign up, so the tie-dye activity was only from 16 to 21 . Just because you're working with, you know, different materials, different chemicals, we didn't necessarily want to give a nine year old, that, you know, that in their own hands and their parents were like oh.. ( dog barks)

Madi: Sorry, my dogs barking.

James: You're fine. Oh, they are conscious enough to drive a car they're conscious enough to take some time and.

Madi: (dog barking) Gosh, I don't know if you can hear her but she's been really loud. I'm sorry.

James: Nah, you're good.

Madi: Somebody must be home. Okay, so one of the main things that I've been looking at specifically for my lit review is that social media really allows extension to reach new audiences. So even though we've been talking mostly about connecting with the members and the clientele that already exist. But do you think that social media, especially implementing it during covid has allowed you to reach new audiences? People that maybe didn't know what extension was or weren't previous $4 \mathrm{H}$ members that kind of thing.

James: Yeah, absolutely. I would, I know of, I was just thinking as you were talking. I know of probably four or five families that have brought in probably 11 members, because some of them have multiples that saw that we were offering programs and said "hey, Is it too late to enroll?" I think that kind of. I think that that type that type of kind of recruitment is rare for us. Just because usually we aren't kind of the in person. Here's a flyer, you know, we go to schools we take branded information. The kid goes home and says hey this is what I did and $4 \mathrm{H}$ made it happen or extension made it happen. That's kind of where we usually get our hook in. But I would say with covid, this has definitely helped us gain a couple of those families. And it was just kind of an interesting process. Because, not necessarily did they address that as how they 
were you know they were saying, "awh, well my friend.," And I'm going to use the names and, you know, they are real but it's not going to matter to you. So Rachel had three kids enrolled in $4 \mathrm{H}$, her friend Hillary did not ever know about $4 \mathrm{H}$, came from a very kind of suburban type inner city school, so they knew that $4 \mathrm{H}$ was an option in a different state. So when they came here and you know, they're seeing all these posts about Rachel's kids doing tie-dye and then doing camp in a box and then doing you know all these different stem kits and, you know, so on and so forth. She was posting them not only to our page but she was actually sharing on her personal page, so that there proved that you know that kind of little bit of recruitment that was happening, just by that one family saying hey, I saw my friend post x y z. Now we're interested in $4 \mathrm{H}$ because of it. Do I think there could be a future of it? Absolutely. Was it our number one goal? No, I know, usually I use the model. Survive not thrive. And I think that kind of applied very well to this year, because we just wanted to make sure that we were able to retain the level of engagement that we had had previously, but do it on that modified delivery kind of scope.

Madi: (nods) Remain that.. like keep that connection

James: You just froze for a second it, Madi.

Madi: It froze for me too. I was just saying to keep the connection and remain connected with the clientele, is the big thing which is perfect because that's what I'm researching.

James: Exactly. It does come full circle, I know that. We've had a lot of success. I call them one and done programs, I, you know, it's just easier for me to label it something and that's what I do. But it hasn't just been kids we also do teacher buttons, where teachers from Berkeley County, or any staff I should say that employed by the Board of Education, could request a button that way they could wear it with their mask, and we only send it out by Facebook, we didn't send it out any other way. Because we didn't really have the teacher list from the spring that we would normally have for the fall. So we put it out, and it probably had every bit of 1200 likes, shares, you know, reactions. And we ended up making over 300 buttons.

Madi: Yeah.

James: So I mean, we're completely, you know, at the bay of what our capabilities are really on it. Like, as soon as covid hit in March, we had already planned some in person activities, it's kind of around March Madness. So it got completely shook up, as everything else did. So we ended up switching to an online kind of activity where we made brackets out of $4 \mathrm{H}$ songs and sent them out, and actually only know this because I just did the report on it a couple weeks, actually, like, three days ago. But we, I think, total we had 28,000 reactions.

Madi: Wow

James: What started as a, you know, fun activity for our 700 for each members. I think we had something like 2600 brackets filled out from kids all across the state. So I think we were over 30 counties in the state of West Virginia, that we had someone send in a bracket. And it wasn't. It was funny because it was, like I said, completely started for our kids. Ended up being kids from every, you know, 33 counties. We had agents and other counties do it, we had specialists at the 
state level do it. So it was just kind of a really cool activity that kind of blossomed into this huge activity that we weren't planning for but we were able to pick up some really cool sponsors. Gave away, I think we gave away like 25 large prizes like sports chairs, 4H kind of hoodie packages- that gets a hoodie, a t-shirt, a water bottle, a cinch bag, a book or, you know, something, there was something else in there. But it was five things stuffed inside of a $4 \mathrm{H}$ book bag. We give away some $4 \mathrm{H}$ hammocks. You know, it's all about the catch so are grand, those were our grand prizes, we gave away five pack- five of those large scale projects. And then we also did something that was like swinging in, or you know April's coming, April swinging in or something. And we gave away five hammocks. Anything that we could kind of do with those funny, you know, aha moments to kind of keep them engaged, but also to realize like hey, you know there's some pretty cool prizes in this. We found that a lot of times through social media we try to offer them some type of prize or some type of incentive. It can be really simple as like, I know a couple weeks ago I needed some feedback data for my file. So, the magic thing to do was post it on Facebook. Hey fill out the survey. You know you'll be put in to enter to win a \$5 gift card to, I think it was Dunkin Donuts or chick fil a or something like that. But you know within a couple hours I had 100 people sign in and it was perfect.

Madi: Yep. So I think that when you guys did the march madness that was one of my favorite things. I saw that and I was like this is so cool. And it was, it was right at the beginning a covid so I think it. I think it really helps set the tone for your county office.

James: Yes, absolutely. Well it was funny because, it truly started as just a "okay I have to get some Berkeley County kids engaged" For the month of February we had our county roundup so they just finished their posters and pictures. You know, their speech contest, that kind of stuff. and I would like to have some type of activity every month. And without, what's not being able to host our kind of March Madness night. We are planning on bringing everyone in. We rented out one of the local gymnasiums, the kids can play basketball, we had a spot where we could watch the game. We had people bring food. To be able to flip that and still have them engaged in something, you know, it didn't. I don't wanna say it didn't take a lot of time, it did because we did the initial setup and then flipped it to be on this online get up. But it was really interesting for us to be able to still see, you know, not only wasn't an individual that did their bracket, it was," oh I did my bracket, called, you know, my friend down the road, did you do your bracket." So I think the highlight of that for me. So, I was an extension champion instructor, we call them ECI's. So essentially, I got to travel around the state and conduct camp for in 2012, one of the gentleman that I met in Putnam County was a brand new counselor at that time. So, I, of course, Facebook, or he Facebook friend me something kind of, someone posted that way. But she saw the post. And he picks it up and he actually sent it to his group. It's from state $4 \mathrm{H}$ camp, and that's just a group of kids that you get kind of to reflect and talk about different things. So it was really interesting for him to, you know, see it from Putnam County, share it with his grow group from state camp. Those, that was 13 different people, mostly from different counties. And then, you know, was really quick at that point to make that spider, you know, start here, touch different places. But it was really cool to see the end result. Every week, they would kind of, I don't want to say reunite, but kind of have a local reunion about it and kind of talk about what songs got knocked out and complain about what songs got knocked out. But it was nice to hear that it didn't just live on Facebook that it sparked them to actually talk in person, and keep that momentum and keep that kind of communication. And I think that was kind of.. this kind of sounds, I don't 
know what you would call it, but it was kind of a real beautiful thing, because it kind of took initiative to kind of spread back out, although we couldn't be there in person to you know have that physical kind of meaning, you know, it kind of brought back that sense of, hey this is what $4 \mathrm{H}$ is. This is how we reunite with $4 \mathrm{H}$, this is things we talked about, but it was nice to be able to build those connections, even if it was just, you know, started with computers started with, you know, a simple bracket.

Madi: Yeah, well it's one of those things where it's like, it's kind of impossible to make something this great in person just as successful online, and then you do it and you're like, "oh wow like this, this actually worked and people enjoyed it" so yeah I get it completely because I mean of course I saw the post and was participating and liking my favorite song too so it was kind of fun to like look for it and wait for it when there's new ones posted and see like, oh, people liked that song better, like really?

James: It was one of those things where you know they. It took a week or so to gain momentum but once it did, we did not have to promote it. We would make one post, and it would stay in a newsfeed, because there's so many different people sharing it. And that's kind of how we figured out, and it actually was able to go, I think it was to like seven different states, Just, and it's not necessarily that it went to a different state $4 \mathrm{H}$ programs, it went to different states because of the 4H alumni that were from West Virginia. So it was a really cool kind of, again, it just brought people together and a time where we couldn't be together. So that was the best effect that it could have.

Madi: Do you plan on doing it again this March?

James: We are, we are looking at, ha my voice kind of went up there. We are but it is going to be a little bit different. So we're actually going to send out a survey. In February, to, for you to vote on your top, I want to say we did 32 songs, our $4 \mathrm{H}$ kind of song book has about 77 songs in it total. And last year, the younger audience was able to kind of do the voting. So some of the older kind of tried and true songs didn't necessarily make it but a lot of the newer, kind of, upbeat songs did.

Madi: Yes.

James: At some point I thought there was going to be like an anarchy about it but luckily we made it through. So this year, what we're going to do is we're going to send it out and you're actually going to, or not you, whoever wants to do it, will type in their favorite 10 songs. And of course we have the list, broken up by fast, slow, fun, and traditional that they can pick from. And then we're going to come back, you know, compile all those results, all of those votes and we're taking the top 32, and that's what we will start with.

Madi: Okay. And are you going to do, so if you have three two and there's like, let's say there's like eight per category, are you going to take like the top eight per category is just how you're going to break that down.

James: No, so we're actually just going to take the top 32 total. 
Madi: Okay

James: And then we will kind of, even last year that's what we kind of did was took the top 32 and kind of adjusted, a couple of the regions to make them fit. So if you look there, there's a, there's like a hippo song. That was kind of in the last two or three years kind of brand new. And it actually got put over in the traditional section, which normally would not belong there ever. But, you know, again, when we sent it out originally, most of our younger kids had their parents respond for them and you could tell, because our older kids were like "we've never sang that at camp." So that was kind of a really interesting kind of thing we learned was what population we were actually reaching because early on, I think it was all of the nine and 11, nine to 11 year olds, but now I think it has shifted, because of the kind of what they've learned through covid is we post a lot more often than what we would have last year at this time. So they're actually watching it a lot more. So I'm really hoping that this year. It doesn't happen again but if it does, I think that's a very good teachable moment. But yeah, so we're doing it a little bit different. It just kind of makes me laugh when I look back at it and you know, to know that the hippo song beat out Dem bones, kind of blows my mind. Yeah, But it was because none of the older kids. Put third.

Madi: Yeah, which I mean hopefully will change for this coming year because everyone will be ready for it and ready for the survey.

James: Well they know that is what happened, and they are irate I think. Normally I wouldn't use such a harsh word but I think irate is perfect for this.

Madi: Yeah. That's really funny. Well I'm definitely looking forward to that because that was one of my, my favorite things that I saw on Facebook that you all posted so, excited about that. We have a few more questions we've been talking so much that I have to pay attention to, like, what have we already talked about what happened. So if you could pick just like one overarching thing. What would it be for, what do you want your clientele to gain from what you're posting like what's the overarching purpose, like, of your postings on social media.

Jamesi: I would say accessibility. Like I said, it is truly cut down the turnaround time to get information out. For example, we've had a couple of different kind of collections. We've done a food collection. We've done, We're actually just now doing a toy collection. We've done school collections, we've done all kinds of stuff. And I think the ability for us to get that information out has been huge. We used to send out a newsletter once a month, and that was the really the big way we were reaching our participants. The problem is, what I send out today isn't going to be, you know, in hand until after Christmas at this point. So, you know, it was. Instead, it was like, okay, we're going to start this, this is what the community said they needed, let's just start it Monday. So we've done some of those kind of quick turnaround. At one point we asked. Usually we host events that run three weeks, so we our school supply drive was three weeks are, you know, toy drive was three weeks, somewhere in the middle, we kind of asked, so we usually typically do those in July. We collect in July last two weeks of July 1 week of August. We started the toy drive the last week of November. In between there, teachers, you know, found that they needed specific cleaning items, we had people that needed masks, people that need a hand 
sanitizer. So I literally just do it up one day. Hey, if you see this out and about, grab one of these and you can send it to us, you know your local hospital and you could pick where you want to get dropped off.

Madi: Yeah.

James: And we only listed like six places that way it wasn't really a burden on us to be able to deliver it. But we ended up getting over like 600 items within two weeks. And it was soley driven by social media.

Madi: And is that drive something that you've done in the past too?

James: That was kind of an off the cuff. We've done, we call them kind of covid response efforts. So, I oversaw our CEOE, which I don't know what stands for but I can get that to you later. But we didn't have an agent in that position so I kind of took on the roles of it. So we had made cookies earlier on in covid and delivered them and I included my business card and it always, always put if you need any other resources, please let me know. Typically those go to teachers so the type of resources they're asking for is curriculum or activities. When you send those to like doctor's office and fireplaces you get completely different answers, and that's going to do something to fill kind of that gap. And it was kind of just off the web we were sitting, so Fridays are very laid back in our office. So we were sitting there on a Friday and I was like, you know these people have contacted me this week, what do we have what can we order. And we're like, oh, let's just do a drive, and I threw it up on Monday, and the next Friday is when we delivered stuff, so it was a very quick turnaround. The only thing I ended up costing us, it was, I mean was time and gas money. One of our local food stores, Martin's food stores, saw it, they're based out of Pennsylvania, one of the managers contacted me and said hey do you need something to get them to and I was like yeah I'll take whatever, and they actually gave us brand new reusable bags that could be wiped down. So we've kind of used some of those quick turnaround pieces out of the blue and they've still been successful.

Madi: Yeah, which is awesome. Well my reason for asking about if you had done it before covid was I was you know trying to vision like how, you know, you said it's more accessible now being able to use social media so I'm trying to think of something that you did before social media that maybe if you did a food drive beforehand, you wouldn't have gotten that many people because of having to send it on a newsletter and it getting lost in the mail and people throwing it on the counter, forgetting about it.

James: Absolutely

Madi: So I'm trying to think of that comparison like, you know, how much more do you get?

James: And I don't think that exists, to be honest, because we usually set pretty good parameters for what we want. So for example, I always set a goal with 500 toys for a toy drive, we usually get exactly 500 or 600 toys.

Madi: So you just, you keep asking for it until you've reached your goal basically? 
James: And once we reach the goal, we're done sharing it.

Madi: And that makes that makes sense. Because you don't need too much.

James: Well, The reason for it is then it becomes a facilitation. So, I guarantee you, The official cutoff date for toys this Friday. I meet with the local schools on Monday to distribute. I guarantee you I will have toys show up Tuesday. And the problem is then, there,you know, there's no way to get them to kids, there's no way to get them back to the schools, the turnaround rate is. It takes about a week. So for us to be able to get the toys to the kids takes a week for us to get the toy, you know, for us to get the supplies the teachers takes about a week. And some of that's just limited manpower. Because with covid, especially now, we have to wipe everything down we have to sanitize, we have to let it sit for three days before we can actually give it to the schools. So if a toy comes in like Tuesday, by the time it sits for three days after sanitizing, it'll, won't be ready until Friday.

Madi: Yeah, So you got it time it out perfectly.

James: Yeah, that's an interesting battle is what it is.

Madi: Yeah, a new, a new thing that you have to adapt to.

Madi: So thinking about how you use social media now. Can you think of any specific ways that it's been changing since you've been implementing it and how you're adapting to that as social media usage for work has been changing.

James: Oh, absolutely. I think it's been one continuous change since we started. Um Because I think we learn, we learn from each post. For example, our posts used to be, you know, it would literally be a paragraph of information. And we gotten feedback all this is a lot. Can you just put, you know, when the, you know, I want to call it like the" when where why how" kind of aspects of it. And we kind of put that up in a picture or we put the text stuff and show a nice picture, go to a different place for details. And that's what we're doing now. We're at the beginning of covid, It was literally just blocks of text, blocks of information, blocks of go here do this. So we've kind of learned what people are actually stopping and looking at. And that's kind of been through trial and error with leaders and different volunteers and different parent groups as to what is actually being done. But I would say that's been the biggest thing we've learned, and I've changed. As you know, how we are posting, how we construct posts. Because if you look early on we didn't have a large number like respond. But now we're starting to get to the point where, you know, parents anticipate posts from us. They're used to seeing them a certain format. So that is kind of the new standard that we've kind of created.

Madi: And do you foresee any like challenges, so any changes that might be challenges to overcome in the future.

James: Um. I don't think. No, I honestly don't think we'll have any challenges. I think we have an existing challenge of trying to reach a population of people that do not have Facebook. The 
younger adults 16 to 21 have kind of moved away from Facebook so they have Instagram and, you know, TikTok and all these different things and the university is not necessarily adopting these new practices fast enough for us to implement them. So I think we are missing that population. What do foresee see coming up as an issue? I think from here it's just growing. Because, now that we have trained. I don't say that we've trained them but, you know, now they expect to look on Facebook to know when tag in is. They expect to look on Facebook to know when our events start. It's no longer a question of where can I find this information, it's just on the website. Even on you know our Facebook posts we're linking them back to the website, so we're. Again, I think it's just kind of adapting what we have to make it successful.

Madi: And have you thought about possibly making it Instagram and kind of putting posts on there too for maybe the younger kids that don't have Facebook or not sure?

James: Um, we would like to, like I said, we just have to wait for WVU kind of our branding and our communications department to give us the go ahead for it. Yeah, there are some kind of bootleg copies of bootlegs additions of, you know, Instagram and that kind of stuff out there. But because we're held to such a high standard with the land grant university, you know with the real kind of communications department at the view. It's hard for us to do it and not get in trouble for it. So kind of Facebook is the approved way to do it. Instagram is kind of hit or miss. Whether you can do it or not and TikTok is absolute no.

Madi: Oh, no way. Yeah.

James: So we have asked about Snapchat. The problem is how people can respond back. There's no way for us to trace and log, who has been there and who hasn't been in contact. And what they've actually shared, so there's no, there's no tracing our accountability there.

Madi: Yeah, I think Facebook is definitely like the most professional one, therefore it kind of fits the criteria for making that, but maybe I mean maybe because Facebook is being used so much more for things like this maybe the kids that don't have it will actually start using Facebook and get it for these purposes too because I know like you know extension specifically is not the only know what it's like industry or business organization is using it now because of covid specifically so maybe it'll become more popular again.

James: And I think it's just becoming such a routine thing for parents to know to go there for information that we no longer have to force it. So, all like when we advertise stuff, it automatically goes on our Facebook and newsweets. And it's pretty much covers the entire basis. There's not a whole lot of follow up we have to do outside of that. Facebook kind of helps us with that recruitment piece. Newsweets, make sure that we have everyone that's registered. So it's been nice to be able to post it twice versus put it on our newsletter, put it on our website, put it on you know the multitude of different places we have it. It's been nice just to be able to kind of (lost connection)

Madi: All right, I think this is my last question before we wrap up, but do you think that you can like. Do you consider yourself an innovator in regards to utilizing social media as an extension agent? Would you consider yourself an innovator? 
James: Um, I would say yes, based on how we use it. You know, we kind of have gotten creative with how we're reaching them. I know I keep going back to camp but that was probably our most successful virtual program. I don't think there were a lot of other counties that were implementing it quite like we did, because we were very detailed and complex on how we made sure every child was able to access. And we've actually had a lot of people reach out and be like hey, how did you do, you know, how did you share your schedule. How did you share your links. And literally, it was just huge posts. You know, some of those finer details, where we you know we had nine cabin meetings going on at once. Actually we had 18 because we had an older and a younger, but it was, you know, it was kind of interesting to be able to post it all in one place. And, you know, what boys cabin are you in? First it was boys or girls, go to which one you want. And then it was, pick a cabin, 1234, or 12345, You click on it and that's where you're at. We didn't require them to go to the same cabin every night. So it was nice for them to be able to.. most of them stayed in the cabin they chosen the first night. Yeah, but but it was nice for them to have that kind of availability to go off and do those separate events. I think we are innovative in the way that we are sharing the activity. I think a lot of them are still just kind of, we're one of the largest counties, they all, there is in the state for $4 \mathrm{H}$. I think we're in the top five. But it was, it's nice to be able to connect with them and share projects, and you know share and highlight different stories we've done some projects, kind of highlights we've done some book highlights we've done volunteer highlights. But it's being able to still have that connection with $4 \mathrm{H}$, that we don't have because we're not in person. And I think that we've been very innovative, as far as what we're posting. We usually run programs no longer than six weeks total. So that's kind of where our March Madness. That's why we picked the number that I did because it took exactly six weeks. Yeah, anything beyond that. And people are tired of looking at it. And that's just kind of though trial and error, I guess, maybe not all trial and error from this year but it's definitely been a learning point that is climax with 2020 .

Madi: And do you think that even if so let's say covid ends, the virus ends, we're back to in person. Because of all you've been doing on social media, during covid you're going to have to probably continue doing that because it's kind of what the client is used to at this point?

James: Yup. I think making that migration from checking our website. First, has been extremely beneficial because our website is quite daunting, you click the wrong link and you're taking to the state page you click the right link and you know, you hit that target, but it can be a little bit harder to find just because of everything else that is on our website. so being able to pull and extract those individual pieces, I think, will continue regardless of covid or not.

Madi: Which is awesome.

James: Yes, because I think we're doing a better job of reaching our community where they are, versus making our community come to us, even if it is just a click of a website, you know, it still takes a, it takes amount of time. So if you can see it on Facebook and have all the details and not necessarily have to go fishing for it, $i$ think is a lot better. And I think that's why we put on there kind of the leading views of, you know, when, where, what is it, it's enough information that as they are really interested. They have a basis to start on, and they can go right into zsweets and register or they can call the office and getting put on the list, you know, active as an active 
participant. But I think it has definitely put us in a positive place compared to where we could have been without it.

Madi: Awesome. And as we're wrapping up we have just a couple more minutes, but do you have anything else that you want to add that we didn't get to talk about like any cool things that you've done anything you can think of, or any questions specifically about my research,

James: I think the coolest thing we learned it in camp, but we've used it since then, is we control if they can share pictures and our comments sections. So we've been doing a lot more of the tie dye Tuesday's or taco Tuesdays or 4H Fridays, small little kind of spirit days to keep our members engaged and then they can send in a picture of them actually doing it. Prior to that we didn't really know how to facilitate that. But with camp, you know with posting and, you know, understanding what moderator controls we had, I think covid has forced us to use these to our advantage. It's forced us to be out of our comfort zone, it's forced us to learn these new things that we can engage our participants to the max level that they can. So I think that's kind of been the silver lining in this all.

Madi: All of those hidden features that you didn't really know existed.

James: Exactly, including data collection because that has saved us so much time. Hours.

Madi: Yeah, that's awesome. But anything else?

James: I think that's good if anything else, give me a holler. 


\section{Kimberly}

Madi: So the first question is, can you describe what you would consider to be beneficial social media practices that you implement for work purposes?

Kimberly: So, I am in my mid 30s, I hate saying that out loud. But when I was growing up social media wasn't what it is today. we were the like MySpace generation. So just on the very edge of all of these things taking place. I got my facebook account in 2005 when I started college, and I, so just a little background on that, So it- It's not something that has been part of my growing up or like it's happened very organically for me. It's something that's a challenge for me to learn. I feel like I'm one generation ahead of the social media generation. So, we have been on Facebook primarily as far as outreach goes and I know that we target our kids through social media but the truth is on Facebook I think we're getting a lot of their parents. And I think it's been very useful. I know it has because parents can communicate in real time very easily with Facebook Messenger directly to my account or to the extension account. I would like to continue to work towards interacting with our kids in a safe way on social media, that's the other reason I feel comfortable with Facebook with parent sort of supervision right now. And that's why I don't have an Instagram set up yet. I'm, I'm thinking about it I'm on the cusp of that but like I said it's not something that's super easy for me I also live in an area with really limited connectivity. WiFi is illegal, and we don't have cell phone service.

Madi: Yep.

Kimberly: So that changes things a little bit. But so far, like our Facebook has been our primary contact with social media. We're starting to do more group email kind of thing. so our new platform on zsuite, and people are getting us to check me on that platform for for each updates. Does that answer the question?

Madi: Yes, perfect. And I think that you mentioned that you're, you're kind of the generation right before social media really hit and I think that most extension agents, especially in the state of West Virginia are also kind of in the same boat, like social media wasn't something that they've known since they were five years old. It's something that came a little later. So I think that for the most part, unless you're, like, a brand new extension agent like and you like your first couple years I think that most people are in a very similar situation.

Kimberly: I read a funny thing last week and it said I remember back when when a whole day without taking a picture of something. And I was like, Oh, that's so funny because you know now our communication so much that happens through photos and communicating those photos on social media. But yeah.

Madi: And like, social media, I mean, for me, it probably became the big thing closer to high school I mean a little bit in middle school but a lot of the kids nowadays I mean, they're in elementary school and they have Instagrams and accounts like that which is crazy to me but that's kind of just the reality of it now. 
Kimberly: For sure it's scary and exciting all at once.

Madi: Yes, positives and negatives, for sure. Yeah. So thinking about your Facebook posts specifically. Are there certain times when your clientele is most engaged? Can you think of a specific example, or a situation or certain things that you post that your clientele is more engaged with and others.

Kimberly: So offering incentives for things is exciting. I'm offering things that have specific time limits on them seem to have better participation. So when we did our camp in a box, which was the at home virtual portion. And we said like, you have to do this by seven o'clock today. if we put deadlines on things our participation increased. When you leave things open ended it seems like people are like, 'oh yeah I'll get back to that' or 'I'll do that' and then they forget about it. Or they lose interest. But when things are like real time and interactive, we had better participation. As far as what time, If it's something that requires parent help, so our younger kids who don't have social media accounts, it's better to do it in the evening after people have been home for a little bit, had dinner. We aim for like that six to eight o'clock window. Um, I don't try to do a whole lot on the weekends because I feel like everybody's trying to take a breath. So we were trying to keep it in like that Monday through Friday, unless it was something where we could be, you know, real time interactive with them. We kind of stray away from the weekend activity.

Madi: Yeah, the work, the work week would be pretty typical for posts I'd say.

Kimberly: I think it's been tough with our kids this year going so much online school that by the time they finished all their online learning and their requirements for that they're burnt out and being on the computer. So we had to make it all really like fun in something that they wanted to participate in, and nothing that felt like regular school.

Madi: And had.. because you're in Pocahontas county, correct?

Kimberly: Right.

Madi: Okay, so have you been most.. Have students mostly been online for most of the year because I know like some counties have been different where they've been more in person than other counties.

Kimberly: And so ours. We were pretty good we were in really good shape actually until this month. As far as covid went. We had two day a week school for the month of September and then we had four day a week school for the month of October, and for two weeks of November and then the third week of November we turned orange and we haven't been back to school since.

Madi: Wow. And have you noticed since kids haven't been back to school and so they're doing virtual school.. Have you noticed, kind of like a decrease? 
Kimberly: For sure.. So we actually kind of changed the way we're, we're trying to reach our kids, and mailing them paper things at this point, versus trying to do too much on social media because of that burnout being online so much. And because it becomes like not I don't want to say discrimination, but we're definitely not providing a platform that all kids can participate in if they're forced to do online. We have a lot of kids who are sitting in the parking lot on a laptop at the library, trying to do their schoolwork. So the last thing they want to do is spend more time in the parking lot of library trying to access the WiFi.

Madi: Yeah, well I think in your county specifically it's probably like a bigger issue too because there's not that service that everyone else has everywhere else like I can just pick up my phone and scroll through Facebook but if you don't have service in the county, which is a huge issue then you have to find somewhere specifically to go access the social media.

Kimberly: for sure and that was actually a conversation I had with our new dean of extension the first time that I met him, because he said oh well if you don't have cell phone service you can just use the wifi. I said well we don't have Wi Fi it's illegal here and he said what do you mean like he had no.. There was no process of, you know how people are living without cell phone or $\mathrm{WiFi}$, it was just something he hadn't even thought existed anymore.

Madi: I think Pocahontas county is probably the only county I know of personally, or the only place that I know a personally, but I know people that grew up there and stuff and it's always like wow like, that's crazy. It's kind of crazy what we take for granted elsewhere.

Kimberly: It's kind of neat to though because people have started coming here for like family vacations intentionally to detox from technology.

Madi: Yes.

Kimberly:So it's kind of become a marketing tool also it's an interesting you know flip on that. Yeah, that's very cool.

Madi: So can you share some key examples of specific times or events that you utilize social media for- to stay connected with clientele during covid? Anything that stands out?

Kimberly: for sure. So we've done some county wide and club specific 4-H zoom meetings. So, what will be a normal monthly meeting we've been able to host it on zoom. And we've had about $50 \%$ participation which for normal club meetings we usually get about $75 \%$ of participants, or participation so I feel like $50 \%$ is pretty good. So we've been able to use that for club meetings county and club or like county wide and just local clubs. And we've done it for our 4-H leaders. We've done some challenges and some games on our social media. We played a game of bring me, where kids would take a photo of themselves with objects and it was kind of just a silly thing but it kept them engaged and gave them some connection to their friends. So that was good I told you we use it for our 4-H camp. We did camp in a box with an online component so if you don't have access to internet, you can still participate, but if you did there was a social media component of that participated. We've used it to get information out. So as far as like our livestock show and sale we use that to post videos of all of the kids with their animal exhibits, to share that with the community in the same way with our ham bacon and eggs sale. And also we 
use social media for. It's been a great. I mean it's a real time way of communicating cancellations and things which unfortunately this year we've had a lot of cancellations that we've needed to get out to people very quickly so we've used it for some real time communication.

Madi: Has your social media usage increased greatly because of covid or were you actively involved in social media prior to covid? Can you kind of explain that a little bit?

Kimberly: So I started as an agent in 2018 and prior to my starting there was no social media connection to our extension office in Pocahontas county they didn't have any, like, Facebook, they didn't have any email blast, communication, nothing. It was all done to the US Postal Service. So, since 2018, we built our Facebook page and it's gaining momentum but you know since it was new, it doesn't have 20,000 followers. Since covid we've more than doubled our communication on Facebook, when I went to pull the reports this year for my faculty file, we've had, I think it was like 100,000 more contacts than the year before.

Madi: Wow.

Kimberly: So definitely and we've increased it and it has given us a vehicle to really connect to some people that we wouldn't have had the same opportunity to find without it.

Madi: Awesome. And can you think of a specific thing that you might have posted or something that you tried that was new that didn't go very well that you thought kind of would have better results?

Kimberly: Oh, sure. And you know, I just like some days it gets you down and you're like, how come no one participated and other days it's like take a deep breath and try to find a way to build off of it. We wanted to do a door decorating contest for national 4-H week. And so we sent out, you know, this flyer on social media and an email and said decorate your door and post your picture to our Facebook account so we can judge them, and no one did it. Um, but I think it went back to that like we posted it and it had a long deadline, a long turnaround, and so it didn't have the energy of something that happened more rapidly.

Madi: You kind of maybe gave it too much time and then people forgot about it and then it was kind of too late at that point well too much time and also I think we've learned that when we provide examples like 'hey decorate the door. This is the door at, you know, Pendleton bank', just to try to show people that other groups are participating already. I think it kind of like drives that spirit of competition in a healthy way. But when we just post like hey here's our challenge and we don't have those examples with photos of people that everybody knows and wants to participate with we sort of lost that connection.

Madi: Yeah. And if you so let's say that you're doing something kind of similar. Do you usually post the pictures as you go or do you post, will you post pictures of actual people do those like typically get like more attention if you like didn't post the results kind of thing.

Kimberly: For sure, the more photos and connections to actual people that our county knows, the more enthusiasm we see behind stuff and a willingness to give it a try attitude. 
Madi: Which which makes sense and I think that, you know, talking to the other extension agents they've said really similar things like their posts with the photos, get a lot of likes and any photos that specifically have people that are well known or that have their friends in it or community members or people that you know, typically get a lot more attention than just a picture of the snow outside your window or whatever it may be.

Kimberly: Definitely. they want to see the smiling faces and anytime that we can get a younger member to share a photo or a video or like an actual 4 Her versus a community project kind of thing. If we get those like high school age $4 \mathrm{H}$ kids to do something, it seems pretty instant that our kids get excited about mimicking their adult peers, you know.

Madi: Yeah.

Kimberly: they see that and they want to be- They want to be like Haley, their $4 \mathrm{H}$ club president, they gravitate towards those kids because they know them and recognize that.

Madi: Yeah. So, looking at your survey results you included that you use YouTube, Padlet, and Facebook. Am I missing anything else?

Kimberly: That's it for now. I think I'm about to pay one of our college kids who's home for spring semester to take us to the next level. And like I said that Instagram account but I'm afraid of, I think, Our next step is going to be to talk to someone who is very current with social media and figure out if they can help us get to that next level, but for now that's all we're doing is YouTube Padlet and Facebook.

Madi: Can you provide me with an example of how you use each like each platform if you use them for different things, or if you posted the same thing on all the platforms, can you kind of describe that to me.

Kimberly: So we've used YouTube for video links. When we did our county livestock show and sale you could see some videos there. We also, with camp in a box, had some video links to like a frog hunt so we had a going deeper video, you know, you could do the frog hunt with just the paper that supported it or you could go deeper and watch the video link that was available on YouTube. As far as Padlet goes, it's just a baby in this county at this point. It's more of a bulletin board where we just try and get some information out there so that people have access to it but I don't find it to be very interactive at this point- more of just like a kind of a storage space for information that kids could access. My fear was using Padlet too much, is that our new zsuite platform also offers the same kind of bulletin board experience. I do like that it's safer than using Facebook because we can isolate it and, you know, not make a private facebook page more so it's a private Padlet page that always stays there. Yeah, and Facebook. Facebook is Facebook we use it for all of our updates, any information that we're going to send out on like a postcard mailing, we try to post on Facebook also. And then we use that for camp programs and our livestock stuff. I don't know I'm trying to think of what else we use Facebook for. Any of our updates, anytime anyone like has an award or an accomplishment or newspaper article, we link all that into our Facebook. 
Madi: Does your Facebook kind of does it kind of like duplicate your other means of communication. so like let's say that you're sending out an email to inform members or parents or whoever of this, do you typically post that to Facebook as well? Or do it on both? Are they typically separate thing?

Kimberly: We definitely try to make those work together. Like I said with real time stuff Facebook is a lot easier than you know trying to mail out a letter that says our meetings canceled tomorrow. It gives us an opportunity to have things that we can post with a shorter turnaround. But other than that, like when we're sending out, you know it's national for each week where your green shirt on Wednesday, we're going to send that in an email and on our Facebook. So we try to make those things kind of reinforce one another.

Madi And if you- if you had to kind of guess like how many people do you think are on Facebook compared to the attention that you get on sending out the emails and stuff like that because I know your county, specifically probably has a bigger lag in that just because of the lack of Wi Fi and cell phone service.

Madi: Um Do you think there's a pretty big gap between who's on Facebook and the audience you're trying to reach?

Kimberly:I think our Facebook is a wider net. And our emails are more concentrated for people who we know need the information so like our Facebook I think gains community support and maybe you know a parent sees that says oh my kid wanted to join for each like how do I get them in there. So it works more towards recruitment, and it reiterates some of that information like parents who already have kids that are already part of the program, they're going to go on to our Facebook to find whatever update it is, but they, as far as like the difference between the two. When we communicate via email or through are zsuite or in that way we know that we're hitting kids who are already enrolled in the $4 \mathrm{H}$ program.

Madi: And that makes sense too, because I mean you send out an email to who needs that information and then you're putting the Facebook paste- the Facebook post out just kind of to anybody who else might be interested instead sending like the mass email out to everybody, especially because I feel like email sometimes nowadays can get super cluttered too.

So you really want to like narrow down what you need when you send that information out.

Kimberly: I think about when I share a Facebook post too because it could be a Facebook post that like I just thought was interesting or I knew other people needed the information on, I would instantly share it but if that came in an email, I wouldn't forward it to all of my email contacts.

Madi:That's a good point. That definitely makes sense.And one of the, which you kind of touched on this a little bit, which is perfect, but one of the main things I'm looking at is that there's research showing that social media really allows extension to reach those new audiences so extensions able to potentially reach those who are unfamiliar with extension or don't know that extension exists. Can you think of an experience that really supports or denies that statement? 
Kimberly: For sure, um, we advertised for the Mars base camp as the National Youth science data came out of Virginia Tech but we were having facilitators from WVU extension who are teaching it. So we advertise on our Facebook page, and I posted on my personal page. And I actually gained seven new $4 \mathrm{H}$ members because of that advertisement that came just through our social media, and now they're, you know, club members that are going to participate this year, even though that program was in October. it sparked their interest and it, it was a great recruitment tool for us. So we average, last year we have about 160 members. And we're at half this year which I solely blame on covid, but anyways. I'm counting on that being covids faultl. Um, yeah. But so, seven kids is so it's like that's a significant amount of children for us to pick up just because of social media.

Madi: Which is awesome and I think that I'm like thinking about maybe the older kids that really like the traditional extension, you know, and $4 \mathrm{H}$ specifically, I know. I feel like it's harder for the older kids who like the in person camps and that kind of stuff. You know, they get a little worried about you know what, what, what is what is it going to be like with covid, so I think that a lot of that kind of is where maybe you could have possibly lost some of the members in $4 \mathrm{H}$ specifically.

Kimberly: Yeah, I definitely think as covid changes here in the next few months, if we're able to have camp we're going to see our numbers come back up. Camp is such a, like it's kind of a backbone, and I've told people multiple times that camp in a box this past year was like our Hail Mary, and it worked, right for one year, but I don't think it'll work for two years. It could, like, don't get me wrong if it has to it will and will still persevere but kids really really want that in person camping experience and there's just nothing else in my life that I've ever experienced like being out $4 \mathrm{H}$ camp in person.

Madi: Yeah, and I mean extension, especially like it right it relies so heavily on that face to face communication so it's hard to try to implement something without that and I think that throughout the state you all have done a phenomenal job trying to implement that but it definitely is a very, very difficult task.

Kimberly: Yeah, those in person relationships really they keep people's hearts full, and what keeps our volunteers motivated, you know, is to see and hear and experience growth with children.

And when it all is happening virtually, they don't have that same connection and they don't see those same results and so it's hard to continue to motivate volunteers, especially whenever we are doing things electronically and it doesn't require the manpower, you know, one person can lead a zoom meeting with 50 kids. One person can't lead a service activity with 50 kids, so it reduces the need for those people and I think they feel it.

Madi: Yeah. And that's a really good point too that I hadn't thought of.

Kimberly: We're counting on being there once this is over. 
Madi: So can you think of an overarching concept or idea or kind of just like what do you hope that your clientele gains from you implementing social media?

Kimberly: I think it gives them a sense of relief. When we were just doing mailings, they had a lot to keep up with and we deal with a lot of kids live in, you know, more than one home. So mom lives here and dad lives there and my paper mailing went to dads and now I'm at moms and I missed an opportunity. So I think it gives people that sense of like, yeah, I don't know what I did with that paper but go on the Extension Facebook page I'm sure they'll tell you what time it is tonight or like what you were supposed to bring. So I think it kind of gives people a little bit of relief when it comes to having a backup and not just relying on that tangible piece of paper to keep them informed as far as events go.

Madi: Yeah, so it's not like a replacement for the old means it's kind of just like you know an extra cushion and extra means for communication.

Kimberly: Yeah, I definitely just see it as a supplement and I think we still have to you know target all three of those areas because even if we did have the best cell phone service. And the best Wi Fi, we're still trying to reach kids regardless of like their socio economic backgrounds. And so maybe there's one computer in the house or there's no computer in the house, but for some children like that paper mailing is so important and the only way they're going to be informed. So I think it's important to keep all pieces of communication. But I definitely think that the social media aspect of it is creating more community awareness and for our supporters and our donors and our grandparents who were for 4-Hers, I think they love to see it. I think it helps get our newspaper articles more spread further and wider. we have a local county newspaper and it's great and wonderful and everybody who lives here knows it, but it's really great to take those articles and put them on social media and reach my brother North Carolina, or, you know, our 4-H alumni from all over the country.

Madi: Which is awesome and that's that's a huge point because you're reaching you're not just reaching your clientele in your county you're also reaching community and past community members, friends, family of community members, way beyond just Pocahontas County.

Kimberly: Well, and nine times out of ten our stuff is feel-good stuff too, you know, if we have 4-Hers building school gardens and planting pollinator patches and packing shoe boxes for Operation Christmas Child. And so it's information that we're sharing with people that's making people happier. And I think that's so wonderful. And so the broader scope of that, that we can send out in the world, I think is great.

Madi: Yeah. And do you think that so that like talking about you know the feel good posts and stuff have those really increased since covid or did you kind of do that before.

Kimberly:I think they were probably easier to come by before covid, back to that in person component.So like, it's hard to get a picture of everybody who packed a shoe box that they all pack them at their house, versus, we all pack shoe boxes at the Wellness Center and here's a picture of our whole club happy smiling holding a shoe box right. So, easier to capture those things when we were together for sure. And it's also kind of hard to keep that enthusiasm all the 
time, there was a point in the summer, when I talked to the editor of the paper. And I was like, here's what we're doing this week and it was after we had done all of our camp stuff and after we did all of our livestock stuff and she was like, I have to put something in the newspaper that's not $4 \mathrm{H}$ this week, because we were kind of flooding her, you know. And because we were really trying to keep the community morale in spirit up.I might have gone overboard. Is it easier to do it during covid? Definitely not. It was easier to do it before but is it more important right now probably so

Madi: That's a really good point too. do you um.. Can you think of any ways that social media is changing so specifically for implementing it for work purposes. Can you think of how social media is changing and how you have to adapt to this?

Kimberly: So I think it's changing because it's starting to acknowledge that we need safe spaces for kids to communicate on social media that aren't accessible to the greater world.Just like our breakout rooms on zoom that are now available with zoom started. I've been in a zoom classroom for my graduate program for three years now. Zoom was really really basic and now all the sudden we've got breakout rooms and you know we can put people in a waiting room and we can do all kinds of different things on the zoom platform so I see it changing because they're acknowledging that safe spaces or private spaces have to occur. With Facebook I get less of the advertisements to pay for things like I think they're realizing that their free service is pretty important. there for a while it was like hey boost this post for five more dollars. I don't get that as much as I used to. So I see that changing. kind of think of how else I see it. Like I said about the age, you know, the age groups that are targeted. Facebook's not targeting the younger generation and I don't really see them. I heard they're all on Instagram so maybe that's the plan - to target them that way. Yeah. But I don't see them changing as quickly as necessary to kind of draw that younger crowd back in.

Madi: Yeah, and the thing with, you know, Instagram, your, you'd probably be reaching more more kids you've been reaching the younger members. Whereas on Facebook you're reaching the parents but you also have to think about, you know, are the younger members. Do you post the same information to them that you're giving to the parents? Do you have to alter those posts because they're younger and they're using a different platform. And it's like completely different communication methods so there's a lot. There's kind of just like a lot of thought that needs to go into it and I think that because it's such a new thing that a lot of times when you know extension agents are using it or anybody, any organizations that are implementing social media. I think a lot of times it's just trial and error, you got to kind of like figure it out as you go and see what's best for your specific clientele.

Kimberly: For sure, I think we kind of struggle with wanting to target kids but needing their parents and their leaders to be part of the conversation, and I say that, out of.. we had a club meeting that we were allowed to have 25 or less people if everybody wore a mask. So we said no parents only leaders and only 4Hers so that we can have as many features present as possible. Yeah. And so the kids play the whole event. And then they went home and they're like yeah we're doing the Christmas parade is next Friday and parents are like No you're not. Like my eight year old doesn't get to make plans for themselves. So I think I see that through social media we have to be really careful that kids aren't overstepping their authority as children, 
you know, they are still minors and they don't get to make their own plans. So that was something that I definitely want to find a better way to kind of navigate you know to make sure that whatever plans we make can happen with one child participating or the whole entire club but it doesn't. We don't let kids make plans for their parents and you know kind of make that relationship sour that.

Madi: Yeah. Which that is a really hard, a hard thing and it's like you know you get the kids all excited and it's like, well, it might maybe didn't work out completely how you envisioned.

Kimberly: And we've seen that with our like spin clubs, you know, oh I signed up for cooking around the world. Here's the $\$ 30$ of groceries mommy have to go buy them. Well, you know, so just kind of being aware of those things and making sure that we're communicating to not just the kids we're not just the parents but really trying to find a way of keeping everybody engaged in that.

Madi: Yeah. Are there any challenges that you foresee in the future that you will need to overcome in order to keep utilizing the social media platforms that you're using?

Kimberly: Yeah, I mean we have parents who are really uncomfortable with their children being featured on any kind of social media, which is understandable. The problem with it that I see is some parents are really excited to put their children on social media and see them, you know out there in the world. So it creates like, some kids are excluded from that. And when we talk about like awards and being proud of things and we've got everybody's photo but the one child who didn't get to be on social media. Those things kind of create division amongst our kids and embarrassment, you know, and whatever it is that the child who didn't get to participate feels. So I think that's a challenge for sure. Safety is going to be a challenge for us. I live in a rural place in a very safe place thankfully but advertising like we're going to have all the features at the Christmas parade. Well, we just told all of social media that 200 youth, we're going to be at this specific place at this specific time. And that creates a really vulnerable situation. So, trying to have my eyes wide open and not be so like trusting of a platform that I've known since 2005 and realizing, to be very careful with those kinds of things as far as safety goes, and even you know as much as like our $4 \mathrm{~h}$ camp whenever we announced campaigns. I don't know if we should put them out there for the world to know versus in a private email a private mailing. So trying to you know differentiate between what's information that the world can know and what's information that could potentially make our kids more liable to a child abduction kind of thing, or, you know, numerous things that can happen this day and age.

Madi: Do you think it would be helpful if they had maybe if extension came out with more specific guidelines for what to post and whatnot to post I don't know how much, how much guidance you guys have gotten on that but do you think it'd be helpful if there is more specific guidelines for those types of situations where you're kind of not knowing which which direction to lean.

Kimberly: I mean I think always that we can use more training because these things change and adapt so quickly that I'm always open to more and more training and awareness and you know knowledge that I wouldn't even have ever thought of. Because my experience is so different than 
the experience of someone in Pittsburgh, but definitely I think we could use more training in those things. And also I think about like children with broken homes or children with a parent who they have a custody battle with and so back to those liability things being really cautious with that. So yes more training always. Sometimes I don't want to listen to it because I don't want to be on another zoom meeting, but it is necessary.

Madi: Yeah. It's kind of a lot right now, with everything else being online that you're doing to definitely kind of, I think we're everyone's a little burnt out on that online communication aspect,

Kimberly: when we got the save the dates for our spring upcoming zoom meetings and it's like twice a week every week from now until May. I was like, Oh, now I throw my computer in the river I can't do it.

Madi: Yeah. So talking about, you know, having more guidance in the future, what guidance or guidelines do you already have in place for using social media that may be WVU extension provided for you, or anything else of that sort.

Kimberly: Um, I'm not sure about specific guidelines from WVU Extension but I know that we've had some professional development opportunities at some of our annual conferences and we have a state spring conference manual hormones that we all get to attend and I know that those have been topics that have been offered at those events. I'm sure that our media people have something out there but I can't come up with it off the top of my head.

Madi: Yeah. So thinking about social media practices as a whole, for work purposes in the extension office. Would you consider yourself to be an innovator?

Kimberly: I will say yes, and it's easy for me to say that because we had nothing before so a little effort feels like it was innovative. I know that I have a whole lot to learn. And a whole lot of, you know, progress to make trying to make this path forward. Yeah. But yeah, I mean, yes I know we are. I know we've been innovative because we've been able to reach so many people, and those numbers, you know, they're not perfect but they do definitely tell a story of how many people had a connection to Pocahontas county $4-\mathrm{H}$. That wouldn't have had that same connection otherwise.

Madi: Yeah. Can you think of a specific time or situation where you noticed another county or another extension agent, doing something on social media, that made you think, oh hey that's cool, like I wish, or I want to do that or I wish I'd thought of that kind of thing.

Kimberly: Definitely Megan Midcaps in Lewis County and every post that she puts on Facebook is clean and perfect and straight off of publisher. And when I you know type things into the comment box and I pick the like prefab Facebook background color to go with that I'm like, I'm so late. I see like what Megan's dealing with graphics and photos and consistency and just sort of stuff out there. I aspire to that. 
Madi: And I think that's pretty much all my questions but if you have any more information anything that we didn't cover that you really want to add that you think would be beneficial to my resort research, or if you have any questions for me about my research I can do that before we wrap up.

Kimberly: I think I'm good. Good luck with everything it's an exciting time at the end of your, your here. Yeah, semester was almost there is the level if you need anything else for me. If you need anything else for me. You know where to find me on my email and also my cell phones listed in there you can call anytime, and good luck. Thank you.

Madi: I really appreciate it. No problem. Bye. Bye. 


\title{
APPENDIX D
}

\author{
IRB Approval
}

\section{WestVirginiaUniversity. \\ OFFICE OF HUMAN RESEARCH PROTECTIONS \\ sas Onentrut Noge hoad Morpantomn WW asso6}

Acknowledgement of Exemption

$11 / 05 / 2020$

To: Haley Rosson

From: WVU Office of Research Integrity \& Compliance

Protocol Type: Exempt

Submission Type: Initial

Funding: N/A

WVU Protocol \#: 2011160709

Protocol Title: How is WVU Extension Service Utilizing Innovative Social Media Practices to Remain Connected with clientele while adhering to quarantine protocol enacted by COVID-19?

The West Virginia University Institutional Review Board has reviewed your submission of Exempt protocol 2011160709. Additional details regarding the review are below:

- This research study was granted an exemption because the Research involves educational tests, survey procedures, interview procedures or observation of public behavior and (i) information obtained is recorded in such a manner that human subjects cannot be identified, directly or through identifiers linked to the subjects; and (ii) any disclosure of the human subjects responses outside the research could not reasonably place the subjects at risk of criminal or civil liability or be damaging to the subjects financial standing, employability, or reputation [45 CFR 46.101(2)]. All exemptions are only good for three years. If this research extends more than three years beyond the approved date, then the researcher will have to request another exemption. The following documents have been acknowledged for use in this study and are available in the WVU+ke system:

The following documents were reviewed and approved for use as part of this submission. Only the documents listed below may be used in the research. Please access and print the files in the Notes \& Attachments section of your approved protocol.

- Cover Letter Interview docx

Protocol o: 2011160709

FWA: 00005078

NORO: 0000194
Phooe: 304-293-7073

Fax: 304-293-3098

Emsil: IRB\%mail wvu.colu 
- Sample Interview Questions.docx

- Recruitment Email \& Phone Script.docx

WVU IRB acknowledgement of protocol 2011160709 will expire on 11/04/2025.

If the study is to continue beyond the expiration date, a renewal application must be submitted no later than two (2) weeks prior to expiration date. It is your responsibility to submit your protocol for renewal.

Once you begin your human subjects research, the following regulations apply:

1. Unanticipated or serious adverse events and/or side effects encountered in this research study must be reported to the IRB within five (5) days, using the Notify IRB action in the electronic protocol.

2. Any modifications to the study protocol should be submitted only if there will be an increase in risk to subjects accompanying the proposed change(s).

3. You may not use a modified information sheet until it has been reviewed and acknowledged by the WVU IRB prior to implementation.

The Office of Research Integrity and Compliance will be glad to provide assistance to you throughout the research process. Please feel free to contact us by phone, at 304.293 .7073 or by email at IRB@mail.wru.edu.

Sincerely,

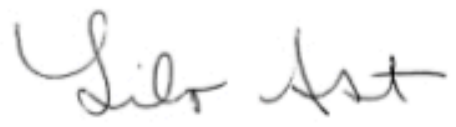

Lile Ast

IRB Administrator 